Supporting Information

\title{
Phosphine-Catalyzed [2 + 4] Annulation of Allenoates with Thiazolone-Derived Alkenes: Synthesis of Functionalized 6,7-Dihydro-5H-pyrano[2,3-d]thiazoles
}

Chang Wang, Zhenzhen Gao, Leijie Zhou, Chunhao Yuan, Zhanhu Sun, Yumei Xiao* and Hongchao Guo

Table of Contents

General Information $\quad$ S1

General Procedure for Synthesis of Substrates 1 and $2 \quad$ S1

General Procedure of $\mathrm{PMe}_{2} \mathrm{Ph}$-catalyzed [2+4] Annulation $\quad \mathrm{S} 1$

General Procedure of Asymmetric [2+4] Annulation $\quad$ S2

Screening of Reaction Conditions for Asymmetric Annulation S2

Characterization Data of Products 3 and (+)-3 S3 - S20

Transformations of the Product 3aa and Preparation on the Gram Scale S20-S21

Copies of ${ }^{1} \mathrm{H}$ and ${ }^{13} \mathrm{C}$ NMR Spectra $\quad$ S22 - S53

HPLC Chromatograms of the Products (+)-3 S54 - S57

$\begin{array}{lr}\text { X-Ray Crystallographic Data of 3ma } & \text { S58 - S67 }\end{array}$ 


\section{General Information}

All reactions were performed under $\mathrm{N}_{2}$ atmospheres in oven-dried glassware with magnetic stirring. Unless otherwise stated, all reagents were purchased from commercial suppliers and used without further purification. All solvents were purified and dried according to standard methods prior to use. Organic solutions were concentrated under reduced pressure on a rotary evaporator or an oil pump. Reactions were monitored through thin layer chromatography (TLC) on silica gelprecoated glass plates. Chromatograms were visualized by fluorescence quenching with UV light at $254 \mathrm{~nm}$. Flash column chromatography was performed using Qingdao Haiyang flash silica gel (200300 mesh). Infrared spectra were recorded using a Bruker Optics TENSOR 27 instrument. ${ }^{1} \mathrm{H}$ and ${ }^{13} \mathrm{C}$ NMR spectra were recorded in $\mathrm{CDCl}_{3}$ using a $300 \mathrm{MHz}$ NMR instrument (referenced internally to $\mathrm{Me}_{4} \mathrm{Si}$ ). $1 \mathrm{H}$ NMR data are reported as follows: chemical shift, multiplicity $(\mathrm{s}=$ singlet; $\mathrm{d}=$ doublet; $\mathrm{q}=$ quartet; $\mathrm{m}=$ multiplet; $\mathrm{br}=$ broad $)$, coupling constant $(\mathrm{Hz})$, and integral. Data for ${ }^{13} \mathrm{C} \mathrm{NMR}$ spectra are reported in terms of chemical shift. Optical rotation was obtained on a Perkin-Elmer 343 polarimeter. Accurate mass measurements were performed using an Agilent instrument with the ESI-MS technique. HPLC analysis was performed on Agilent 1100 series, UV detection monitored at $254 \mathrm{~nm}$, using an $\mathrm{R} \& \mathrm{C}$ RC-OD column with hexane and $i-\mathrm{PrOH}$ as the eluent. X-ray crystallographic data were collected using a MM007HF Saturn724+.

\section{Synthesis of Substrates 1 and 2}

Allenoates 1 were synthesized by previous literature procedure. ${ }^{1}$ Thiazolone-derived alkenes 2 used were prepared by the literature procedure. ${ }^{2}$

\section{General Procedure for Preparation of Racemic Products 3 by [2 + 4] Annulation of Thiazolone-Derived Alkenes 2 with Allenoates 1.}

Under nitrogen atmosphere, to a mixture of thiazolone-derived alkenes $2(0.1 \mathrm{mmol})$ and additive $4 \AA \mathrm{MS}(100 \mathrm{mg})$ in $1 \mathrm{~mL}$ of toluene in a Schlenk tube, $\alpha$-substitued allenoates 1 (1.5 eq., 0.15 mmol) followed with catalyst $\mathrm{PMe}_{2} \mathrm{Ph}(10 \mathrm{~mol} \%, 0.01 \mathrm{mmol})$ were added at $40{ }^{\circ} \mathrm{C}$. The resulting mixture was stirred until the starting material was completely consumed (monitored by TLC) and then was concentrated to dryness. The residue was purified through flash column chromatography (EtOAc/PE) to afford the corresponding cycloaddition product 3.

\footnotetext{
${ }^{1}$ Liu, H.; Liu, Y.; Yuan, C.; Wang, G.-P.; Zhu, S.-F.; Wu, Y.; Wang, B.; Sun, Z.; Xiao, Y.; Zhou, Q. L.; Guo, H. Org. Lett. 2016, 18, 1302.

${ }^{2}$ Lin, L.; Yang, Y.-H.; Wang, M.; Lai, L.-H.; Guo, Y.-R.; Wang, R. Chem. Commun. 2015, 51, 8134.
} 


\section{General Procedure for Asymmetric [2 + 4] Annulation}

Under nitrogen atmosphere, to a mixture of thiazolone-derived alkenes $2(0.05 \mathrm{mmol})$ and additive $4 \AA$ MS (50 mg) in $1 \mathrm{~mL}$ of toluene in a Schlenk tube, $\alpha$-substisuted allenotes 1 (1.5 eq., $0.075 \mathrm{mmol})$ followed with catalyst $\mathbf{P 1}(20 \mathrm{~mol} \%, 0.01 \mathrm{mmol})$ were added at $80^{\circ} \mathrm{C}$. The resulting mixture was stirred until the starting material was completely consumed (monitored by TLC) and then was concentrated to dryness. The residue was purified through flash column chromatography $(\mathrm{EtOAc} / \mathrm{PE})$ to afford the corresponding cycloaddition product $(+)-3$.

Table 1. Optimization of Reaction Conditions for Asymmetric Annulation ${ }^{a}$

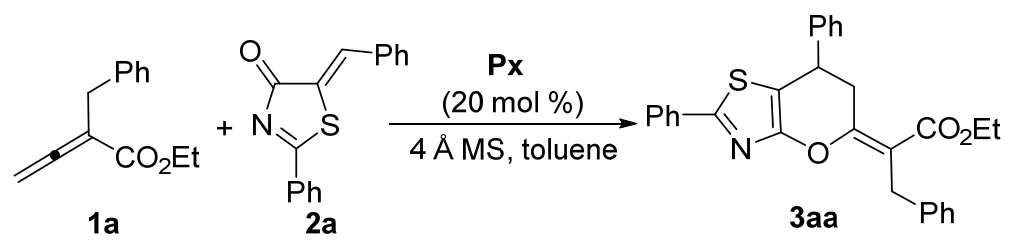<smiles>Fc1ccc(P2CC3CCC2C3)cc1</smiles><smiles>c1ccc2c(P3CCC4CC4C3)cccc2c1</smiles>

P2<smiles></smiles>

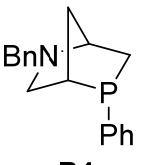<smiles>[Al]C1PC2CC1C2</smiles>

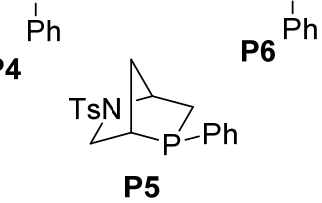<smiles>COc1ccc(P2CC3CCC2C3)cc1</smiles>

P7<smiles>CCC(C)C(Cc1ccccc1)NC(=S)NC(C(=O)NCc1ccccc1)c1ccccc1</smiles>

P8<smiles>CCC(C)NC(=O)C(NC(=S)NC(Cc1ccccc1)C(C)CC)C(=O)NC(C)C</smiles>

P9<smiles>CCC(Cc1ccccc1)NC(=O)c1cc(C(F)(F)F)cc(C(F)(F)F)c1</smiles><smiles>CCCC(Cc1ccccc1)NC(=S)Nc1cc(C(F)(F)F)cc(C(F)(F)F)c1</smiles>

\begin{tabular}{cccccc}
\hline enrty & Px & $t\left({ }^{\circ} \mathrm{C}\right)$ & $t$ & ${\text { yield }(\%)^{b}}^{b}$ & ee $(\%)^{c}$ \\
\hline 1 & P1 & 25 & $3 \mathrm{~d}$ & 62 & $>99$ \\
2 & P2 & 25 & $3 \mathrm{~d}$ & trace & - \\
3 & P3 & 25 & $3 \mathrm{~d}$ & 52 & $>99$ \\
4 & $\mathbf{P 4}$ & 25 & $3 \mathrm{~d}$ & 38 & $>99$ \\
5 & $\mathbf{P 5}$ & 25 & $3 \mathrm{~d}$ & 68 & -88 \\
6 & $\mathbf{P 6}$ & 25 & $3 \mathrm{~d}$ & 25 & $>99$ \\
7 & $\mathbf{P 7}$ & 25 & $3 \mathrm{~d}$ & 40 & $>99$ \\
8 & $\mathbf{P 8}$ & 25 & $3 \mathrm{~d}$ & 40 & 86 \\
9 & $\mathbf{P 9}$ & 25 & $3 \mathrm{~d}$ & 57 & 70 \\
10 & $\mathbf{P 1 0}$ & 25 & $3 \mathrm{~d}$ & 90 & 23 \\
11 & $\mathbf{P 1 1}$ & 25 & $3 \mathrm{~d}$ & trace & - \\
12 & $\mathbf{P 1}$ & 40 & $20 \mathrm{~h}$ & 74 & 99 \\
13 & $\mathbf{P 1}$ & 60 & $6 \mathrm{~h}$ & 77 & 98 \\
14 & $\mathbf{P 1}$ & 80 & $2.5 \mathrm{~h}$ & 80 & 98
\end{tabular}

${ }^{a}$ Unless otherwise stated, all reactions were carried out with $1 \mathbf{a}(0.075 \mathrm{mmol}), 2 \mathrm{a}(0.05 \mathrm{mmol})$, catalyst $(0.01 \mathrm{mmol})$ additive $4 \AA$ MS $(50 \mathrm{mg})$ in solvent $(1 \mathrm{~mL}) .{ }^{b}$ Isolated yield. ${ }^{c}$ Determined by chiral HPLC analysis. 


\section{Characterization Data for the Racemic Products 3}

\section{Ethyl (E)-2-(2,7-diphenyl-6,7-dihydro-5H-pyrano[2,3-d]thiazol-5-ylidene)-3-}

phenylpropanoate (3aa)<smiles>CCOC(=O)/C(Cc1ccccc1)=C1/CC(c2ccccc2)c2sc(-c3ccccc3)nc2O1</smiles>

Prepared according to the general procedure as described above in $80 \%$ yield $(37.4 \mathrm{mg})$. It was purified by flash chromatography $\left(5.0 \%\right.$ EtOAc/PE) to afford a rufous solid. $\mathrm{mp}=82-90{ }^{\circ} \mathrm{C} ;{ }^{1} \mathrm{H}$ NMR $(300$ $\left.\mathrm{MHz} \mathrm{CDCl}_{3}\right) \delta 7.96-7.86(\mathrm{~m}, 2 \mathrm{H}), 7.48-7.40(\mathrm{~m}, 3 \mathrm{H}), 7.35-7.29(\mathrm{~m}, 5 \mathrm{H}), 7.28-7.15(\mathrm{~m}, 5 \mathrm{H}), 4.38$ $-4.28(\mathrm{q}, J=7.1 \mathrm{~Hz}, 1 \mathrm{H}), 4.14-4.04(\mathrm{~m}, 2 \mathrm{H}), 3.97(\mathrm{q}, J=7.1 \mathrm{~Hz}, 2 \mathrm{H}), 3.86-3.76(\mathrm{~m}, 1 \mathrm{H}), 3.61-3.51$ $(\mathrm{m}, 1 \mathrm{H}), 1.16(\mathrm{t}, J=7.1 \mathrm{~Hz}, 3 \mathrm{H}) ;{ }^{13} \mathrm{C} \mathrm{NMR}\left(75 \mathrm{MHz}, \mathrm{CDCl}_{3}\right) \delta 167.6,164.0,157.6,156.7,141.9$, $140.1,133.1,130.1,128.8,128.6,128.5,128.0,127.4,127.2$, 125.7, 125.5 , 114.6, 109.0, 60.3, 37.2, 32.7, 31.7, 13.9; IR (film) $v_{\max } 3029,2981,2378,2345,1704,1632,1601,1556,1495,1454,1361,1275$, 1186, 1155, 1104, 1032, 763, $700 \mathrm{~cm}^{-1}$; HRMS (ESI) calcd for $\mathrm{C}_{29} \mathrm{H}_{26} \mathrm{NO}_{3} \mathrm{~S}^{+}[\mathrm{M}+\mathrm{H}]^{+} 468.1628$, found 468.1638.

\section{Ethyl(E)-3-phenyl-2-(2-phenyl-7-(o-tolyl)-6,7-dihydro-5H-pyrano[2,3-d]thiazol-5-ylidene)}

\section{propanoate (3ab)}<smiles>CCOC(=O)/C(Cc1ccccc1)=C1\CC(c2ccccc2C)c2sc(-c3ccccc3)nc2O1</smiles>

Prepared according to the general procedure as described above in $78 \%$ yield $(37.5 \mathrm{mg})$. It was purified by flash chromatography $(5.0 \% \mathrm{EtOAc} / \mathrm{PE})$ to afford an orange solid. $\mathrm{mp}=92-98{ }^{\circ} \mathrm{C} ;{ }^{1} \mathrm{H}$ NMR $(300$ $\left.\mathrm{MHz}, \mathrm{CDCl}_{3}\right) \delta 7.98-7.90(\mathrm{~m}, 2 \mathrm{H}), 7.49-7.42(\mathrm{~m}, 3 \mathrm{H}), 7.37-7.28(\mathrm{~m}, 4 \mathrm{H}), 7.25-7.12(\mathrm{~m}, 4 \mathrm{H}), 7.08$ $-7.02(\mathrm{~m}, 1 \mathrm{H}), 4.64-4.56(\mathrm{~m}, 1 \mathrm{H}), 4.17-3.95(\mathrm{~m}, 4 \mathrm{H}), 3.81(\mathrm{dd}, J=14.8,5.6 \mathrm{~Hz}, 1 \mathrm{H}), 3.49(\mathrm{dd}, J=$ 14.7, $7.5 \mathrm{~Hz}, 1 \mathrm{H}), 2.52(\mathrm{~s}, 3 \mathrm{H}), 1.17(\mathrm{t}, J=7.1 \mathrm{~Hz}, 3 \mathrm{H}) ;{ }^{13} \mathrm{C} \mathrm{NMR}\left(75 \mathrm{MHz}, \mathrm{CDCl}_{3}\right) \delta 167.4,163.8$, $157.5,157.1,140.0,139.7,134.9,133.0,130.3,129.9,128.6,128.4,127.8,127.0,126.6,126.2,125.5$, 114.6, 108.7, 60.1, 33.2, 31.6, 31.4, 19.1, 13.7; IR (film) $v_{\max } 3027,2979,1704,1639,1556,1494,1461$, $1361,1274,1183,1104,1081,1032,951,761,700,680 \mathrm{~cm}^{-1}$; HRMS (ESI) calcd for $\mathrm{C}_{30} \mathrm{H}_{28} \mathrm{NO}_{3} \mathrm{~S}^{+}$ $[\mathrm{M}+\mathrm{H}]^{+}$482.1784, found 482.1794. 
<smiles>CCOC(=O)/C(Cc1ccccc1)=C1/CC(c2cccc(C)c2)c2sc(-c3ccccc3)nc2O1</smiles>

Prepared according to the general procedure as described above in $83 \%$ yield $(40.0 \mathrm{mg})$. It was purified by flash chromatography $(5.0 \% \mathrm{EtOAc} / \mathrm{PE})$ to afford a rufous solid. $\mathrm{mp}=52-60{ }^{\circ} \mathrm{C} ;{ }^{1} \mathrm{H} \mathrm{NMR}(300$ $\left.\mathrm{MHz}, \mathrm{CDCl}_{3}\right) \delta 7.95-7.87(\mathrm{~m}, 2 \mathrm{H}), 7.48-7.38(\mathrm{~m}, 3 \mathrm{H}), 7.35-7.23(\mathrm{~m}, 4 \mathrm{H}), 7.22-7.10(\mathrm{~m}, 5 \mathrm{H}), 4.33$ $-4.25(\mathrm{~m}, 1 \mathrm{H}), 4.15-3.94(\mathrm{~m}, 4 \mathrm{H}), 3.87-3.77(\mathrm{~m}, 1 \mathrm{H}), 3.55-3.44(\mathrm{~m}, 1 \mathrm{H}), 2.36(\mathrm{~s}, 3 \mathrm{H}), 1.17(\mathrm{t}, J=$ $7.1 \mathrm{~Hz}, 3 \mathrm{H}) ;{ }^{13} \mathrm{C} \mathrm{NMR}\left(75 \mathrm{MHz}, \mathrm{CDCl}_{3}\right) \delta 167.4,163.7,157.6,156.4,140.0,138.8,136.9,133.0,129.9$, $129.1,128.6,128.4,127.8,126.9,125.5,114.4,109.2,60.1,36.7,32.6,31.6,20.7,13.7$; IR (film) $v_{\max }$ $2964,1705,1639,1556,1513,1495,1454,1361,1261,1183,1103,1029,864,800,763,700 \mathrm{~cm}^{-1}$; HRMS (ESI) calcd for $\mathrm{C}_{30} \mathrm{H}_{28} \mathrm{NO}_{3} \mathrm{~S}^{+}[\mathrm{M}+\mathrm{H}]^{+}$482.1784, found 482.1797.

Ethyl (E)-3-phenyl-2-(2-phenyl-7-(p-tolyl)-6,7-dihydro-5H-pyrano[2,3-d]thiazol-5ylidene) $\quad$ propanoate (3ad)<smiles>CCOC(=O)/C(Cc1ccccc1)=C1/CC(c2ccc(C)cc2)c2sc(-c3ccccc3)nc2O1</smiles>

Prepared according to the general procedure as described above in $85 \%$ yield $(40.9 \mathrm{mg})$. It was purified by flash chromatography $(5.0 \% \mathrm{EtOAc} / \mathrm{PE})$ to afford an orange solid. $\mathrm{mp}=102-110{ }^{\circ} \mathrm{C} ;{ }^{1} \mathrm{H} \mathrm{NMR}(300$ $\left.\mathrm{MHz}, \mathrm{CDCl}_{3}\right) \delta 7.94-7.86(\mathrm{~m}, 2 \mathrm{H}), 7.46-7.39(\mathrm{~m}, 3 \mathrm{H}), 7.33-7.28(\mathrm{~m}, 2 \mathrm{H}), 7.27-7.22(\mathrm{~m}, 2 \mathrm{H}), 7.20$ $-7.11(\mathrm{~m}, 5 \mathrm{H}), 4.34-4.25(\mathrm{~m}, 1 \mathrm{H}), 4.15-3.93(\mathrm{~m}, 4 \mathrm{H}), 3.86-3.76(\mathrm{~m}, 1 \mathrm{H}), 3.54-3.43(\mathrm{~m}, 1 \mathrm{H}), 2.36$ $(\mathrm{s}, 3 \mathrm{H}), 1.16(\mathrm{t}, J=7.1 \mathrm{~Hz}, 3 \mathrm{H}) ;{ }^{13} \mathrm{C} \mathrm{NMR}\left(75 \mathrm{MHz}, \mathrm{CDCl}_{3}\right) \delta 167.4,163.7,157.6,156.4,140.0,138.8$, 136.9, 133.0, 129.9, 129.1, 128.6, 128.3, 127.8, 126.9, 125.4, 114.3, 109.2, 60.1, 36.7, 32.6, 31.6, 20.7, 13.7; IR (film) $v_{\max } 3029,2981,1705,1641,1604,1557,1494,1454,1361,1260,1181,1105,1031,913$, 879, 763, $701 \mathrm{~cm}^{-1}$; HRMS (ESI) calcd for $\mathrm{C}_{30} \mathrm{H}_{28} \mathrm{NO}_{3} \mathrm{~S}^{+}[\mathrm{M}+\mathrm{H}]^{+} 482.1784$, found 482.1784 . 
Ethyl (E)-2-(7-(2-methoxyphenyl)-2-phenyl-6,7-dihydro-5H-pyrano[2,3-d]thiazol-5-

ylidene)-3- phenylpropanoate (3ae)<smiles>CCOC(=O)/C(Cc1ccccc1)=C1/CC(c2ccccc2OC)c2sc(-c3ccccc3)nc2O1</smiles>

Prepared according to the general procedure as described above in $78 \%$ yield (38.8 $\mathrm{mg})$. It was purified by flash chromatography $\left(5.0 \%\right.$ EtOAc/PE) to afford a rufous solid. $\mathrm{mp}=62-70{ }^{\circ} \mathrm{C} ;{ }^{1} \mathrm{H} \mathrm{NMR}(300$ $\left.\mathrm{MHz}, \mathrm{CDCl}_{3}\right) \delta 7.96-7.88(\mathrm{~m}, 2 \mathrm{H}), 7.46-7.39(\mathrm{~m}, 3 \mathrm{H}), 7.34-7.22(\mathrm{~m}, 5 \mathrm{H}), 7.21-7.13(\mathrm{~m}, 1 \mathrm{H}), 7.04$ $(\mathrm{dd}, J=7.6,1.7 \mathrm{~Hz}, 1 \mathrm{H}), 6.95-6.84(\mathrm{~m}, 2 \mathrm{H}), 4.79(\mathrm{t}, J=5.9 \mathrm{~Hz}, 1 \mathrm{H}), 4.12-3.93(\mathrm{~m}, 4 \mathrm{H}), 3.92(\mathrm{~s}, 3 \mathrm{H})$, $3.90-3.83(\mathrm{~m}, 1 \mathrm{H}), 3.50(\mathrm{dd}, J=15.0,6.0 \mathrm{~Hz}, 1 \mathrm{H}), 1.16(\mathrm{t}, J=7.1 \mathrm{~Hz}, 3 \mathrm{H}) ;{ }^{13} \mathrm{C} \mathrm{NMR}\left(75 \mathrm{MHz}, \mathrm{CDCl}_{3}\right)$ $\delta 167.4,163.5,158.0,156.7,156.2,140.1,133.1,129.8,129.7,128.6,128.4,128.1,127.7,127.4,125.4$, $125.4,120.4,114.1,110.1,108.4,60.0,55.0,31.6,30.2,30.2,13.7$; IR (film) $v_{\max } 2964,1704,1636$, 1601, 1557, 1493, 1462, 1439, 1361, 1261, 1178, 1104, 1029, 865, 800, 755, $700 \mathrm{~cm}^{-1}$; HRMS (ESI) calcd for $\mathrm{C}_{30} \mathrm{H}_{28} \mathrm{NO}_{4} \mathrm{~S}^{+}[\mathrm{M}+\mathrm{H}]^{+}$498.1734, found 498.1736.

Ethyl (E)-2-(7-(4-methoxyphenyl)-2-phenyl-6,7-dihydro-5H-pyrano[2,3-d]thiazol-5-ylidene)-3phenylpropanoate (3af)<smiles>CCOC(=O)/C(Cc1ccccc1)=C1/CC(c2ccc(OC)cc2)c2sc(-c3ccccc3)nc2O1</smiles>

Prepared according to the general procedure as described above in $81 \%$ yield $(40.3 \mathrm{mg})$. It was purified by flash chromatography $(5.0 \% \mathrm{EtOAc} / \mathrm{PE})$ to afford a rufous solid. $\mathrm{mp}=51-57{ }^{\circ} \mathrm{C} ;{ }^{1} \mathrm{H} \mathrm{NMR}(300$ $\left.\mathrm{MHz}, \mathrm{CDCl}_{3}\right) \delta 7.96-7.87(\mathrm{~m}, 2 \mathrm{H}), 7.49-7.39(\mathrm{~m}, 3 \mathrm{H}), 7.34-7.24(\mathrm{~m}, 4 \mathrm{H}), 7.22-7.11(\mathrm{~m}, 3 \mathrm{H}), 6.91$ $-6.83(\mathrm{~m}, 2 \mathrm{H}), 4.34-4.24(\mathrm{~m}, 1 \mathrm{H}), 4.17-3.94(\mathrm{~m}, 4 \mathrm{H}), 3.81(\mathrm{~s}, 3 \mathrm{H}), 3.80-3.74(\mathrm{~m}, 1 \mathrm{H}), 3.56-3.44$ (m, 1H), $1.17(\mathrm{t}, J=7.1 \mathrm{~Hz}, 3 \mathrm{H}) ;{ }^{13} \mathrm{C} \mathrm{NMR}\left(75 \mathrm{MHz}, \mathrm{CDCl}_{3}\right) \delta 167.4,163.7,158.6,157.6,156.4,140.0$, 133.9, 133.0, 129.9, 128.6, 128.4, 128.0, 127.8, 125.45, 114.4, 113.8, 109.4, 60.1, 54.9, 36.3, 32.8, 31.6, 13.7; IR (film) $v_{\max } 3029,2981,1704,1641,1603,1556,1508,1454,1361,1256,1228,1186,1159$, $1104,1031,835,763,700,678,540 \mathrm{~cm}^{-1}$; HRMS (ESI) calcd for $\mathrm{C}_{30} \mathrm{H}_{28} \mathrm{NO}_{4} \mathrm{~S}^{+}[\mathrm{M}+\mathrm{H}]^{+} 498.1734$, found 498.1734. 
Ethyl (E)-2-(7-(4-fluorophenyl)-2-phenyl-6,7-dihydro-5H-pyrano[2,3-d]thiazol-5-ylidene)-3phenylpropanoate (3ag)<smiles>CCOC(=O)/C(Cc1ccccc1)=C1/CC(c2ccc(F)cc2)c2sc(-c3ccccc3)nc2O1</smiles>

Prepared according to the general procedure as described above in $85 \%$ yield $(41.3 \mathrm{mg})$. It was purified by flash chromatography $(5.0 \% \mathrm{EtOAc} / \mathrm{PE})$ to afford an orange solid. $\mathrm{mp}=88-97{ }^{\circ} \mathrm{C} ;{ }^{1} \mathrm{H}$ NMR $(300$ $\left.\mathrm{MHz}, \mathrm{CDCl}_{3}\right) \delta 7.95-7.88(\mathrm{~m}, 2 \mathrm{H}), 7.48-7.39(\mathrm{~m}, 3 \mathrm{H}), 7.32-7.23(\mathrm{~m}, 4 \mathrm{H}), 7.21-7.13(\mathrm{~m}, 3 \mathrm{H}), 7.05$ - 6.96(m, 2H), $4.38-4.28(\mathrm{~m}, 1 \mathrm{H}), 4.15-3.90(\mathrm{~m}, 4 \mathrm{H}), 3.77-3.65(\mathrm{~m}, 1 \mathrm{H}), 3.64-3.52(\mathrm{~m}, 1 \mathrm{H}), 1.16$ (t, $J=7.1 \mathrm{~Hz}, 3 \mathrm{H}) ;{ }^{13} \mathrm{C}$ NMR $\left(75 \mathrm{MHz}, \mathrm{CDCl}_{3}\right) \delta 167.3,164.0,161.8$ (d, $\left.J=246.1 \mathrm{~Hz}\right), 157.08,156.6$, $139.9,137.5$ (d, $J=3.2 \mathrm{~Hz}), 132.9,130.0,128.6,128.5,128.3,127.8,125.5$ (d, $J=2.9 \mathrm{~Hz}), 115.4,115.1$, 114.8, 108.4, 60.1, 36.3, 32.6, 31.5, 13.7; IR (film) $v_{\max } 2980,2837,1704,1636,1611,1557,1512,1455$, $1360,1251,1179,1104,1033,953,830,763,737,701,678,547 \mathrm{~cm}^{-1}$; HRMS (ESI) calcd for $\mathrm{C}_{29} \mathrm{H}_{25} \mathrm{FNO}_{3} \mathrm{~S}^{+}[\mathrm{M}+\mathrm{H}]^{+} 486.1534$, found 486.1537 .

\section{Ethyl(E)-2-(7-(2-chlorophenyl)-2-phenyl-6,7-dihydro-5H-pyrano[2,3-d]thiazol-5-ylidene)-3-} phenylpropanoate (3ah)<smiles>CCOC(=O)/C(Cc1ccccc1)=C1/CC(c2ccccc2Cl)c2sc(-c3ccccc3)nc2O1</smiles>

Prepared according to the general procedure as described above in $90 \%$ yield $(45.0 \mathrm{mg})$. It was purified by flash chromatography $(5.0 \% \mathrm{EtOAc} / \mathrm{PE})$ to afford an orange solid. $\mathrm{mp}=79-85^{\circ} \mathrm{C} ;{ }^{1} \mathrm{H}$ NMR $(300$ $\left.\mathrm{MHz}, \mathrm{CDCl}_{3}\right) \delta 8.01-7.91(\mathrm{~m}, 2 \mathrm{H}), 7.52-7.40(\mathrm{~m}, 4 \mathrm{H}), 7.36-7.11(\mathrm{~m}, 7 \mathrm{H}), 7.03-6.97(\mathrm{~m}, 1 \mathrm{H}), 4.95$ $-4.86(\mathrm{~m}, 1 \mathrm{H}), 4.15-4.03(\mathrm{~m}, 3 \mathrm{H}), 4.00-3.89(\mathrm{~m}, 2 \mathrm{H}), 3.54-3.42(\mathrm{~m}, 1 \mathrm{H}), 1.17$ (t, J= $7.1 \mathrm{~Hz}, 3 \mathrm{H})$; ${ }^{13} \mathrm{C}$ NMR $\left(75 \mathrm{MHz}, \mathrm{CDCl}_{3}\right) \delta$ 167.2, 164.3, 157.3, 156.4, 139.9, 138.8, 132.9, 132.7, 130.1, 129.4, 128.6, 128.4, 128.4, 128.3, 127.8, 126.8, 125.5, 125.5, 115.4, 106.6, 60.2, 33.4, 31.7, 30.6, 13.7; IR (film) $v_{\max }$ $3028,2980,1705,1640,1556,1495,1442,1362,1275,1184,1104,1078,1036,965,760,699,675 \mathrm{~cm}^{-}$ ${ }^{1}$; HRMS (ESI) calcd for $\mathrm{C}_{29} \mathrm{H}_{25} \mathrm{ClNO}_{3} \mathrm{~S}^{+}[\mathrm{M}+\mathrm{H}]^{+} 502.1238$, found 502.1235 . 
Ethyl(E)-2-(7-(4-chlorophenyl)-2-phenyl-6,7-dihydro-5H-pyrano[2,3-d]thiazol-5-ylidene)-3phenylpropanoate (3ai)<smiles>CCOC(=O)/C(Cc1ccccc1)=C1/CC(c2ccc(Cl)cc2)c2sc(-c3ccccc3)nc2O1</smiles>

Prepared according to the general procedure as described above in $92 \%$ yield (46.2 $\mathrm{mg}$ ). It was purified by flash chromatography $(5.0 \% \mathrm{EtOAc} / \mathrm{PE})$ to afford a rufous solid. $\mathrm{mp}=51-58{ }^{\circ} \mathrm{C} ;{ }^{1} \mathrm{H} \mathrm{NMR}(300$ $\left.\mathrm{MHz}, \mathrm{CDCl}_{3}\right) \delta 7.95-7.87(\mathrm{~m}, 2 \mathrm{H}), 7.48-7.40(\mathrm{~m}, 3 \mathrm{H}), 7.32-7.25(\mathrm{~m}, 6 \mathrm{H}), 7.21-7.10(\mathrm{~m}, 3 \mathrm{H}), 4.36$ $-4.26(\mathrm{~m}, 1 \mathrm{H}), 4.15-3.90(\mathrm{~m}, 4 \mathrm{H}), 3.74-3.56(\mathrm{~m}, 2 \mathrm{H}), 1.16(\mathrm{t}, J=7.1 \mathrm{~Hz}, 3 \mathrm{H}) ;{ }^{13} \mathrm{C} \mathrm{NMR}(75 \mathrm{MHz}$, $\left.\mathrm{CDCl}_{3}\right) \delta 167.3,164.1,156.9,156.6,140.3,139.9,133.0,132.9,130.1,128.63,128.60,128.4,128.3$, 127.8, 125.6, 125.5, 115.0, 107.9, 60.2, 36.4, 32.4, 31.5, 13.7; IR (film) $v_{\max } 3028,2981,1704,1640$, 1556, 1492, 1454, 1410, 1361, 1256, 1182, 1104, 1031, 1015, 827, 762, $688 \mathrm{~cm}^{-1}$; HRMS (ESI) calcd for $\mathrm{C}_{29} \mathrm{H}_{25} \mathrm{ClNO}_{3} \mathrm{~S}^{+}[\mathrm{M}+\mathrm{H}]^{+}$502.1238, found 502.1245.

\section{Ethyl (E)-2-(7-(4-bromophenyl)-2-phenyl-6,7-dihydro-5H-pyrano[2,3-d]thiazol-5-}

ylidene)-3- phenylpropanoate (3aj)<smiles>CCOC(=O)/C(Cc1ccccc1)=C1/CC(c2ccc(Br)cc2)c2sc(-c3ccccc3)nc2O1</smiles>

Prepared according to the general procedure as described above in $92 \%$ yield $(50.1 \mathrm{mg})$. It was purified by flash chromatography $\left(5.0 \%\right.$ EtOAc/PE) to afford a rufous solid. $\mathrm{mp}=57-65{ }^{\circ} \mathrm{C} ;{ }^{1} \mathrm{H} \mathrm{NMR}(300$ $\left.\mathrm{MHz}, \mathrm{CDCl}_{3}\right) \delta 7.95-7.88(\mathrm{~m}, 2 \mathrm{H}), 7.47-7.40(\mathrm{~m}, 5 \mathrm{H}), 7.31-7.23(\mathrm{~m}, 4 \mathrm{H}), 7.21-7.14(\mathrm{~m}, 1 \mathrm{H}), 7.11$ $-7.05(\mathrm{~m}, 2 \mathrm{H}), 4.35-4.26(\mathrm{~m}, 1 \mathrm{H}), 4.15-3.91(\mathrm{~m}, 4 \mathrm{H}), 3.74-3.56(\mathrm{~m}, 2 \mathrm{H}), 1.16(\mathrm{t}, J=7.1 \mathrm{~Hz}, 3 \mathrm{H})$; ${ }^{13} \mathrm{C}$ NMR $\left(75 \mathrm{MHz}, \mathrm{CDCl}_{3}\right) \delta 167.3,164.1,156.9,156.6,140.8,139.8,132.8,131.6,130.1,128.7,128.6$, $128.3,127.8,125.5,125.5,121.1,115.0,107.78,60.2,36.5,32.3,31.5,13.7$; IR (film) $v_{\max } 3028,2980$, $1704,1640,1556,1489,1454,1407,1361,1274,1182,1105,1074,1031,1011,953,880,822,762,737$, $700 \mathrm{~cm}^{-1}$; HRMS (ESI) calcd for $\mathrm{C}_{29} \mathrm{H}_{25} \mathrm{BrNO}_{3} \mathrm{~S}^{+}[\mathrm{M}+\mathrm{H}]^{+}$546.0733, found 546.0743. 
Ethyl (E)-2-(7-(4-cyanophenyl)-2-phenyl-6,7-dihydro-5H-pyrano[2,3-d]thiazol-5-ylidene)-3phenylpropanoate (3ak)<smiles>CCOC(=O)/C(Cc1ccccc1)=C1\CC(c2ccc(C#N)cc2)c2sc(-c3ccccc3)nc2O1</smiles>

Prepared according to the general procedure as described above in $82 \%$ yield ( $40.4 \mathrm{mg})$. It was purified by flash chromatography $(5.0 \% \mathrm{EtOAc} / \mathrm{PE})$ to afford a rufous solid. $\mathrm{mp}=57-61{ }^{\circ} \mathrm{C} ;{ }^{1} \mathrm{H} \mathrm{NMR}(300$ $\left.\mathrm{MHz}, \mathrm{CDCl}_{3}\right) \delta 7.95-7.88(\mathrm{~m}, 2 \mathrm{H}), 7.61-7.54(\mathrm{~m}, 2 \mathrm{H}), 7.48-7.41(\mathrm{~m}, 3 \mathrm{H}), 7.31-7.24(\mathrm{~m}, 6 \mathrm{H}), 7.23$ $-7.14(\mathrm{~m}, 1 \mathrm{H}), 4.45-4.35(\mathrm{~m}, 1 \mathrm{H}), 4.15-3.88(\mathrm{~m}, 4 \mathrm{H}), 3.84-3.73(\mathrm{~m}, 1 \mathrm{H}), 3.64-3,52(\mathrm{~m}, 1 \mathrm{H}), 1.15$ $(\mathrm{t}, J=7.1 \mathrm{~Hz}, 3 \mathrm{H}) ;{ }^{13} \mathrm{C} \mathrm{NMR}\left(75 \mathrm{MHz}, \mathrm{CDCl}_{3}\right) \delta 167.1,164.6,156.8,156.2,147.0,139.7,132.7,132.2$, 130.3, 128.7, 128.3, 127.8, 125.6, 125.5, 118.2, 115.6, 111.1, 106.3, 60.3, 36.9, 31.9, 31.5, 13.7; IR (film) $v_{\max } 2964,2346,2229,1704,1641,1608,1556,1502,1454,1414,1362,1260,1178,1104,1030,800$, 763, 690, 661, $555 \mathrm{~cm}^{-1}$; HRMS (ESI) calcd for $\mathrm{C}_{30} \mathrm{H}_{25} \mathrm{~N}_{2} \mathrm{O}_{3} \mathrm{~S}^{+}[\mathrm{M}+\mathrm{H}]^{+}$493.1580, found 493.1581.

Ethyl (E)-2-(7-(3,4-dichlorophenyl)-2-phenyl-6,7-dihydro-5H-pyrano[2,3-d]thiazol-5-ylidene)-3phenylpropanoate (3al)<smiles>CCOC(=O)/C(Cc1ccccc1)=C1\CC(c2ccc(Cl)c(Cl)c2)c2sc(-c3ccccc3)nc2O1</smiles>

Prepared according to the general procedure as described above in $75 \%$ yield $(40.1 \mathrm{mg})$. It was purified by flash chromatography $(5.0 \% \mathrm{EtOAc} / \mathrm{PE})$ to afford a rufous solid. $\mathrm{mp}=58-61{ }^{\circ} \mathrm{C} ;{ }^{1} \mathrm{H} \mathrm{NMR}(300$ $\left.\mathrm{MHz}, \mathrm{CDCl}_{3}\right) \delta 7.96-7.87(\mathrm{~m}, 2 \mathrm{H}), 7.51-7.41(\mathrm{~m}, 3 \mathrm{H}), 7.39-7.32(\mathrm{~m}, 2 \mathrm{H}), 7.29-7.14(\mathrm{~m}, 5 \mathrm{H}), 7.05$ - $6.99(\mathrm{~m}, 1 \mathrm{H}), 4.36-4.26(\mathrm{~m}, 1 \mathrm{H}), 4.15-3.89(\mathrm{~m}, 4 \mathrm{H}), 3.75-3.57(\mathrm{~m}, 2 \mathrm{H}), 1.16(\mathrm{t}, J=7.1 \mathrm{~Hz}, 3 \mathrm{H})$; ${ }^{13} \mathrm{C}$ NMR $\left(75 \mathrm{MHz}, \mathrm{CDCl}_{3}\right) \delta 167.2,164.5,156.7,156.3,142.0,139.7,132.7,132.5,131.3,130.4,130.2$, 129.0, 128.7, 128.2, 127.9, 126.4, 125.6, 125.5, 115.3, 106.9, 60.3, 36.2, 32.1, 31.5, 13.67; IR (film) $v_{\max }$ $2927,1704,1640,1555,1495,1470,1400,1361,1275,1188,1104,1031,820,763,701,663,470 \mathrm{~cm}^{-}$ 1; HRMS (ESI) calcd for $\mathrm{C}_{29} \mathrm{H}_{24} \mathrm{Cl}_{2} \mathrm{NO}_{3} \mathrm{~S}^{+}[\mathrm{M}+\mathrm{H}]^{+}$536.0848, found 536.0847. 
Ethyl(E)-2-(7-(naphthalen-2-yl)-2-phenyl-6,7-dihydro-5H-pyrano[2,3-d]thiazol-5-ylidene)-3phenylpropanoate (3am)<smiles>CCOC(=O)/C(Cc1ccccc1)=C1/CC(c2ccc3ccccc3c2)c2sc(-c3ccccc3)nc2O1</smiles>

Prepared according to the general procedure as described above in $77 \%$ yield $(40.0 \mathrm{mg})$. It was purified by flash chromatography $(5.0 \% \mathrm{EtOAc} / \mathrm{PE})$ to afford an orange solid. $\mathrm{mp}=120-125{ }^{\circ} \mathrm{C} ;{ }^{1} \mathrm{H} \mathrm{NMR}(300$ $\left.\mathrm{MHz}, \mathrm{CDCl}_{3}\right) \delta 7.97-7.90(\mathrm{~m}, 2 \mathrm{H}), 7.88-7.75(\mathrm{~m}, 3 \mathrm{H}), 7.69-7.66(\mathrm{~m}, 1 \mathrm{H}), 7.54-7.47(\mathrm{~m}, 2 \mathrm{H}), 7.47$ $-7.41(\mathrm{~m}, 3 \mathrm{H}), 7.40-7.35(\mathrm{~m}, 1 \mathrm{H}), 7.32-7.15(\mathrm{~m}, 5 \mathrm{H}), 4.57-4.45(\mathrm{~m}, 1 \mathrm{H}), 4.14-3.94(\mathrm{~m}, 4 \mathrm{H}), 3.90$ $-3.80(\mathrm{~m}, 1 \mathrm{H}), 3.78-3.68(\mathrm{~m}, 1 \mathrm{H}), 1.11(\mathrm{t}, J=7.1 \mathrm{~Hz}, 3 \mathrm{H}) ;{ }^{13} \mathrm{C} \mathrm{NMR}\left(75 \mathrm{MHz}, \mathrm{CDCl}_{3}\right) \delta 167.4,164.0$, $157.4,156.6,139.9,139.1,133.1,133.0,132.5,130.0,128.6,128.4,128.3,127.8,127.6,127.4,126.0$, $125.8,125.7,125.5,125.0,114.6,108.6,60.1,37.2,32.4,31.6,13.6$; IR (film) $v_{\max } 3027,2980,1704$, $1636,1602,1556,1495,1454,1358,1271,1179,1104,1031,957,858,818,749,700,678,479 \mathrm{~cm}^{-1}$; HRMS (ESI) calcd for $\mathrm{C}_{33} \mathrm{H}_{28} \mathrm{NO}_{3} \mathrm{~S}^{+}[\mathrm{M}+\mathrm{H}]^{+}$518.1784, found 518.1784.

\section{Ethyl (E)-3-phenyl-2-(2-phenyl-7-(thiophen-2-yl)-6,7-dihydro-5H-pyrano[2,3-d]thiazol-5-} ylidene) propanoate (3an)<smiles>CCOC(=O)/C(Cc1ccccc1)=C1/CC(c2cccs2)c2sc(-c3ccccc3)nc2O1</smiles>

Prepared according to the general procedure as described above in $70 \%$ yield $(33.1 \mathrm{mg})$. It was purified by flash chromatography $\left(5.0 \%\right.$ EtOAc/PE) to afford a rufous solid. $\mathrm{mp}=92-96{ }^{\circ} \mathrm{C} ;{ }^{1} \mathrm{H}$ NMR $(300$ $\left.\mathrm{MHz}, \mathrm{CDCl}_{3}\right) \delta 7.99-7.90(\mathrm{~m}, 2 \mathrm{H}), 7.51-7.42(\mathrm{~m}, 3 \mathrm{H}), 7.35-7.16(\mathrm{~m}, 6 \mathrm{H}), 7.02-6.91(\mathrm{~m}, 2 \mathrm{H}), 4.75$ $-4.61(\mathrm{~m}, 1 \mathrm{H}), 4.25-3.95(\mathrm{~m}, 4 \mathrm{H}), 3.88-3.71(\mathrm{~m}, 2 \mathrm{H}), 1.22(\mathrm{t}, J=7.1 \mathrm{~Hz}, 3 \mathrm{H}) ;{ }^{13} \mathrm{C} \mathrm{NMR}(75 \mathrm{MHz}$, $\left.\mathrm{CDCl}_{3}\right) \delta 167.3,163.9,156.8,156.0,145.1,139.8,132.9,130.0,128.6,128.3,127.8,126.5,125.5,125.5$, 124.6, 124.3, 115.0, 108.6, 60.2, 32.7, 32.4, 31.6, 13.7; IR (film) $v_{\max } 3062,3028,2980,1704,1640$, $1557,1495,1453,1359,1271,1179,1105,1031,960,915,849,762,699 \mathrm{~cm}^{-1}$; HRMS (ESI) calcd for $\mathrm{C}_{27} \mathrm{H}_{24} \mathrm{NO}_{3} \mathrm{~S}_{2}^{+}[\mathrm{M}+\mathrm{H}]^{+}$474.1153, found 474.1198. 
<smiles>CCOC(=O)/C(Cc1cccc(C)c1)=C1/CC(c2ccccc2)c2sc(-c3ccccc3)nc2O1</smiles>

Prepared according to the general procedure as described above in $62 \%$ yield $(29.9 \mathrm{mg})$. It was purified by flash chromatography $(5.0 \% \mathrm{EtOAc} / \mathrm{PE})$ to afford an orange solid. $\mathrm{mp}=96-100{ }^{\circ} \mathrm{C} ;{ }^{1} \mathrm{H} \mathrm{NMR}(300$ $\left.\mathrm{MHz} \mathrm{CDCl}_{3}\right) \delta 7.97-7.86(\mathrm{~m}, 2 \mathrm{H}), 7.47-7.39(\mathrm{~m}, 3 \mathrm{H}), 7.37-7.28(\mathrm{~m}, 3 \mathrm{H}), 7.26-6.96(\mathrm{~m}, 6 \mathrm{H}), 4.37$ $-4,29(\mathrm{~m}, 1 \mathrm{H}), 4.13-4.03(\mathrm{~m}, 2 \mathrm{H}), 3.96$ (q, J=7.1 Hz, 2H), $3.84-3.72(\mathrm{~m}, 1 \mathrm{H}), 3.61-3.49(\mathrm{~m}, 1 \mathrm{H})$, $2.32(\mathrm{~s}, 3 \mathrm{H}), 1.16(\mathrm{t}, J=7.1 \mathrm{~Hz}, 3 \mathrm{H}) ;{ }^{13} \mathrm{C} \mathrm{NMR}\left(75 \mathrm{MHz}, \mathrm{CDCl}_{3}\right) \delta 167.6,164.0,157.4,156.8,142.0$, 140.0 , 137.3 , 133.1 , 130.1 , 129.4 , 128.8 , 128.6 , 127.8 , 127.3 , 127.1 , 126.4 , $125.6,125.6,114.8$, 108.9 , 60.2 , 37.3 , 32.7 , 31.6 , 21.3 , 13.7 ; IR (film) $v_{\max }$ 2980, 2379, 2346, 1705, 1636, 1605, 1557 , 1494, 1455, 1361, 1258, 1188, 1160, 1105, 1069, 1034, 952, 763, 736, 700, $681 \mathrm{~cm}^{-1}$; HRMS (ESI) calcd for $\mathrm{C}_{30} \mathrm{H}_{28} \mathrm{NO}_{3} \mathrm{~S}^{+}[\mathrm{M}+\mathrm{H}]^{+}$482.1784, found 482.1799 .

Ethyl (E)-2-(2,7-diphenyl-6,7-dihydro-5H-pyrano[2,3-d]thiazol-5-ylidene)-3-(p-tolyl) propanoate (3ca)<smiles>CCOC(=O)/C(Cc1ccc(C)cc1)=C1/CC(c2ccccc2)c2sc(-c3ccccc3)nc2O1</smiles>

Prepared according to the general procedure as described above in $67 \%$ yield $(32.3 \mathrm{mg})$. It was purified by flash chromatography $(5.0 \% \mathrm{EtOAc} / \mathrm{PE})$ to afford a refous solid. $\mathrm{mp}=62-67{ }^{\circ} \mathrm{C} ;{ }^{1} \mathrm{H} \mathrm{NMR}(300$ $\left.\mathrm{MHz}, \mathrm{CDCl}_{3}\right) \delta 7.96-7.87(\mathrm{~m}, 2 \mathrm{H}), 7.47-7.38(\mathrm{~m}, 3 \mathrm{H}), 7.38-7.28(\mathrm{~m}, 3 \mathrm{H}), 7.27-7.17(\mathrm{~m}, 4 \mathrm{H}), 7.12$ - $7.03(\mathrm{~m}, 2 \mathrm{H}), 4.38-4.29(\mathrm{~m}, 1 \mathrm{H}), 4.15-4.04(\mathrm{~m}, 2 \mathrm{H}), 5.02-4.92(\mathrm{~m}, 2 \mathrm{H}), 3.84-3.76(\mathrm{~m}, 1 \mathrm{H}), 3.56$ $-3.48(\mathrm{~m}, 1 \mathrm{H}), 2.32(\mathrm{~s}, 3 \mathrm{H}), 1.18(\mathrm{t}, J=7.1 \mathrm{~Hz}, 3 \mathrm{H}) ;{ }^{13} \mathrm{C} \mathrm{NMR}\left(75 \mathrm{MHz}, \mathrm{CDCl}_{3}\right) \delta 167.6,163.9,157.3$, $156.8,142.0,137.0,135.0,133.1,130.1,128.7,128.6,128.6,128.4,127.4,127.2,125.6,114.9,108.9$, 60.2, 37.3, 32.8, 31.3, 20.8, 13.9; IR (film) $v_{\max } 2980,2345,1705,1641,1603,1556,1514,1502,1455$, 1362, 1258, 1185, 1108, 1069, 1033, 788, 763, 737, $700 \mathrm{~cm}^{-1}$; HRMS (ESI) calcd for $\mathrm{C}_{30} \mathrm{H}_{28} \mathrm{NO}_{3} \mathrm{~S}^{+}$ $[\mathrm{M}+\mathrm{H}]^{+} 482.1784$, found 482.1791 . 
Ethyl (E)-3-(4-(tert-butyl)phenyl)-2-(2,7-diphenyl-6,7-dihydro-5H-pyrano[2,3-d]thiazol-5-ylidene) propanoate (3da)<smiles>CCOC(=O)/C(Cc1ccc(C(C)(C)C)cc1)=C1/CC(c2ccccc2)c2sc(-c3ccccc3)nc2O1</smiles>

Prepared according to the general procedure as described above in $71 \%$ yield $(37.2 \mathrm{mg})$. It was purified by flash chromatography $\left(5.0 \%\right.$ EtOAc/PE) to afford an orange solid. $\mathrm{mp}=100-110{ }^{\circ} \mathrm{C} ;{ }^{1} \mathrm{H}$ NMR (300 MHz, $\left.\mathrm{CDCl}_{3}\right) \delta 7.95-7.85(\mathrm{~m}, 2 \mathrm{H}), 7.48-7.39(\mathrm{~m}, 3 \mathrm{H}), 7.37-7.28(\mathrm{~m}, 5 \mathrm{H}), 7.28-7.37$ - $7.27(\mathrm{~m}, 4 \mathrm{H}), 4.33(\mathrm{~m}, 1 \mathrm{H}), 4.16-4.06(\mathrm{~m}, 2 \mathrm{H}), 4.06-3.90(\mathrm{~m}, 2 \mathrm{H}), 3.85-3.75(\mathrm{~m}, 1 \mathrm{H}), 3.55-$ $3.45(\mathrm{~m}, 1 \mathrm{H}), 1.32(\mathrm{~s}, 9 \mathrm{H}), 1.18(\mathrm{t}, J=7.1 \mathrm{~Hz}, 3 \mathrm{H}) ;{ }^{13} \mathrm{C} \mathrm{NMR}\left(75 \mathrm{MHz}, \mathrm{CDCl}_{3}\right) \delta 167.7,163.9,157.4$, 156.8, 148.4, 142.0, 137.0, 133.2, 130.1, 128.7, 128.6, 128.2, 127.3, 127.2, 125.6, 124.9, 114.9, 109.0, 60.2, 37.3, 34.1, 32.8, 31.24, 31.18, 13.9; IR (film) $v_{\max } 3029,2963,1705,1639,1556,1502,1455$, 1361, 1258, 1184, 1118, 1101, 1070, 1033, 963, 807, 762, 738, 700, $556 \mathrm{~cm}^{-1}$; HRMS (ESI) calcd for $\mathrm{C}_{33} \mathrm{H}_{34} \mathrm{NO}_{3} \mathrm{~S}^{+}[\mathrm{M}+\mathrm{H}]^{+}$524.2254, found 524.2266.

Ethyl $\quad(E)-3-(3,5-d i m e t h o x y p h e n y l)-2-(2,7-d i p h e n y l-6,7-d i h y d r o-5 H$-pyrano[2,3-d]thiazol -5-ylidene)propanoate (3ea)<smiles>CCOC(=O)C(Cc1cc(OC)cc(OC)c1)=C1CC(c2ccccc2)c2sc(-c3ccccc3)nc2O1</smiles>

Prepared according to the general procedure as described above in $70 \%$ yield $(37.0 \mathrm{mg})$. It was purified by flash chromatography $(5.0 \% \mathrm{EtOAc} / \mathrm{PE})$ to afford a rufous solid. $\mathrm{mp}=120-126{ }^{\circ} \mathrm{C} ;{ }^{1} \mathrm{H}$ NMR $(300$ $\left.\mathrm{MHz}, \mathrm{CDCl}_{3}\right) \delta 7.92-7.83(\mathrm{~m}, 2 \mathrm{H}), 7.45-7.37$ (m, 3H), $7.34-7.26(\mathrm{~m}, 3 \mathrm{H}), 7.22-7.16(\mathrm{~m}, 2 \mathrm{H}), 6.54$ $-6.49(\mathrm{~m}, 2 \mathrm{H}), 6.31-6.27(\mathrm{~m}, 1 \mathrm{H}), 4.35-4.26(\mathrm{~m}, 1 \mathrm{H}), 4.15-4.05(\mathrm{~m}, 2 \mathrm{H}), 3.98-3.86(\mathrm{~m}, 2 \mathrm{H}), 3.83$ $-3.76(\mathrm{~m}, 1 \mathrm{H}), 3.76-3.70(\mathrm{~m}, 6 \mathrm{H}), 3.53-3.43(\mathrm{~m}, 1 \mathrm{H}), 1.18(\mathrm{t}, J=7.1 \mathrm{~Hz}, 3 \mathrm{H}) ;{ }^{13} \mathrm{C}$ NMR $(75 \mathrm{MHz}$, $\left.\mathrm{CDCl}_{3}\right) \delta 167.3,163.8,160.2,157.6,156.5,142.4,141.8,132.92,130.0,128.6,128.5,127.2,127.0$, 125.4, 114.4, 108.8, 106.5, 98.0, 60.2, 54.9, 37.1, 32.6, 31.8, 13.8; IR (film) $v_{\max } 2936,2837,1704,1636$, 1596, 1556, 1501, 1459, 1429, 1361, 1290, 1255, 1193, 1156, 1103, 1070, 1032, 830, 763, $701 \mathrm{~cm}^{-1}$; HRMS (ESI) calcd for $\mathrm{C}_{31} \mathrm{H}_{30} \mathrm{NO}_{5} \mathrm{~S}^{+}[\mathrm{M}+\mathrm{H}]^{+}$528.1784, found 528.1752 . 
Ethyl (E)-2-(2,7-diphenyl-6,7-dihydro-5H-pyrano[2,3-d]thiazol-5-ylidene)-3-(2-fluorophenyl) propanoate (3fa)<smiles>CCOC(=O)C(Cc1ccccc1F)=C1CC(c2ccccc2)c2sc(-c3ccccc3)nc2O1</smiles>

Prepared according to the general procedure as described above in $71 \%$ yield $(34.5 \mathrm{mg})$. It was purified by flash chromatography $\left(5.0 \%\right.$ EtOAc/PE) to afford a rufous solid. $\mathrm{mp}=40-45{ }^{\circ} \mathrm{C} ;{ }^{1} \mathrm{H}$ NMR $(300$ $\left.\mathrm{MHz}, \mathrm{CDCl}_{3}\right) \delta 7.96-7.86(\mathrm{~m}, 2 \mathrm{H}), 7.48-7.40(\mathrm{~m}, 3 \mathrm{H}), 7.40-7.28$ (m, 4H), $7.26-6.97$ (m, 5H), 4.41 $-4.32(\mathrm{~m}, 1 \mathrm{H}), 4.15-3.98(\mathrm{~m}, 4 \mathrm{H}), 3.86-3.77(\mathrm{~m}, 1 \mathrm{H}), 3.67-3.57$ (m, 1H), $1.12(\mathrm{t}, J=7.1 \mathrm{~Hz}, 3 \mathrm{H})$; ${ }^{13} \mathrm{C}$ NMR $\left(75 \mathrm{MHz}, \mathrm{CDCl}_{3}\right) \delta 167.3,164.0,160.9$ (d, $\left.J=243.8 \mathrm{~Hz}\right), 158.2,156.6,141.9,133.1,130.1$, $129.9(\mathrm{~d}, J=4.5 \mathrm{~Hz}), 128.8,128.6,127.4,127.3,127.2,126.9,126.7,125.6,123.5(\mathrm{~d}, J=3.0 \mathrm{~Hz}), 114.9$, 114.6, 113.0, 108.9, 60.3, 37.1, 32.7, 24.8, 13.7; IR (film) $v_{\max } 2965,2345,1705,1640,1586,1556,1491$, $1455,1362,1261,1230,1179,1108,1033,801,760,700 \mathrm{~cm}^{-1}$; HRMS (ESI) calcd for $\mathrm{C}_{29} \mathrm{H}_{25} \mathrm{FNO}_{3} \mathrm{~S}^{+}$ $[\mathrm{M}+\mathrm{H}]^{+} 486.1534$, found 486.1540 .

\section{Ethyl (E)-2-(2,7-diphenyl-6,7-dihydro-5H-pyrano[2,3-d]thiazol-5-ylidene)-3-(3-fluorophenyl)} propanoate (3ga)<smiles>CCOC(=O)/C(Cc1cccc(F)c1)=C1/CC(c2ccccc2)c2sc(-c3ccccc3)nc2O1</smiles>

Prepared according to the general procedure as described above in $72 \%$ yield $(35.0 \mathrm{mg})$. It was purified by flash chromatography $(5.0 \% \mathrm{EtOAc} / \mathrm{PE})$ to afford a rufous solid. mp $=41-47{ }^{\circ} \mathrm{C} ;{ }^{1} \mathrm{H}$ NMR $(300$ $\left.\mathrm{MHz}, \mathrm{CDCl}_{3}\right) \delta 7.99-7.84(\mathrm{~m}, 2 \mathrm{H}), 7.49-7.40$ (m, 3H), $7.38-7.29$ (m, 3H), $7.26-7.05$ (m, 4H), 7.05 - $6.82(\mathrm{~m}, 2 \mathrm{H}), 4.39-4.29(\mathrm{~m}, 1 \mathrm{H}), 4.16-3.89(\mathrm{~m}, 4 \mathrm{H}), 3.85-3.73(\mathrm{~m}, 1 \mathrm{H}), 3.66-3.55(\mathrm{~m}, 1 \mathrm{H}), 1.16$ $(\mathrm{t}, J=7.1 \mathrm{~Hz}, 3 \mathrm{H}) ;{ }^{13} \mathrm{C} \mathrm{NMR}\left(75 \mathrm{MHz}, \mathrm{CDCl}_{3}\right) \delta 167.3,164.1,162.7$ (d, $\left.J=243.7 \mathrm{~Hz}\right), 158.2,156.5$, $142.8,142.7,141.8,133.1,130.1,129.3,129.2,128.8,128.6,127.4,127.1,125.6,124.1,115.3$ (d, $J=$ $13.5 \mathrm{~Hz}), 113.9,112.5$ (d, $J=21.0 \mathrm{~Hz}), 108.9,60.3,37.1,32.7,31.5,13.9$; IR (film) $v_{\max } 2981,2381$, $1705,1636,1615,1588,1557,1487,1453,1361,1300,1258,1187,1136,1105,1032,964,866,763$, $738,700 \mathrm{~cm}^{-1}$; HRMS (ESI) calcd for $\mathrm{C}_{29} \mathrm{H}_{25} \mathrm{FNO}_{3} \mathrm{~S}^{+}[\mathrm{M}+\mathrm{H}]^{+} 486.1534$, found 486.1540 . 
<smiles>CCOC(=O)/C(Cc1ccc(F)cc1)=C1/CC(c2ccccc2)c2sc(-c3ccccc3)nc2O1</smiles>

Prepared according to the general procedure as described above in 75\% yield (36.4 mg). It was purified by flash chromatography $(5.0 \% \mathrm{EtOAc} / \mathrm{PE})$ to afford an orange solid. $\mathrm{mp}=101-106{ }^{\circ} \mathrm{C} ;{ }^{1} \mathrm{H} \mathrm{NMR}(300$ $\left.\mathrm{MHz}, \mathrm{CDCl}_{3}\right) \delta 7.99-7.84(\mathrm{~m}, 2 \mathrm{H}), 7.49-7.40(\mathrm{~m}, 3 \mathrm{H}), 7.38-7.29(\mathrm{~m}, 3 \mathrm{H}), 7.26-7.05(\mathrm{~m}, 4 \mathrm{H}), 7.05$ $-6.82(\mathrm{~m}, 2 \mathrm{H}), 4.39-4.29(\mathrm{~m}, 1 \mathrm{H}), 4.16-3.89(\mathrm{~m}, 4 \mathrm{H}), 3.85-3.73(\mathrm{~m}, 1 \mathrm{H}), 3.66-3.55(\mathrm{~m}, 1 \mathrm{H}), 1.16$ $(\mathrm{t}, J=7.1 \mathrm{~Hz}, 3 \mathrm{H}) ;{ }^{13} \mathrm{C} \mathrm{NMR}\left(75 \mathrm{MHz}, \mathrm{CDCl}_{3}\right) \delta 167.3,164.1,158.2,156.5,142.8,142.7,141.8,133.1$, $129.3,129.2,128.77(\mathrm{~d}, J=204.0 \mathrm{~Hz}) 128.75,128.6,127.4,127.1,125.6,124.1,115.3(\mathrm{~d}, J=13.5 \mathrm{~Hz})$, 113.9, 112.5 (d, $J=21.0 \mathrm{~Hz}), 108.9,60.3,37.1,32.7,31.5,13.6$; IR (film) $v_{\max } 2981,2365,2346,1705$, 1636, 1597, 1557, 1501, 1455, 1431, 1361, 1301, 1260, 1186, 1105, 1033, 763, $700 \mathrm{~cm}^{-1}$; HRMS (ESI) calcd for $\mathrm{C}_{29} \mathrm{H}_{25} \mathrm{FNO}_{3} \mathrm{~S}^{+}[\mathrm{M}+\mathrm{H}]^{+} 486.1534$, found 486.1538 .

\section{Ethyl (E)-3-(2-chlorophenyl)-2-(2,7-diphenyl-6,7-dihydro-5H-pyrano[2,3-d]thiazol-5-ylidene) propanoate (3ia)}<smiles>CCOC(=O)C(Cc1ccccc1Cl)=C1CC(c2ccccc2)c2sc(-c3ccccc3)nc2O1</smiles>

Prepared according to the general procedure as described above in $70 \%$ yield $(35.0 \mathrm{mg})$. It was purified by flash chromatography $\left(5.0 \%\right.$ EtOAc/PE) to afford an orange solid. mp $=88-92{ }^{\circ} \mathrm{C} ;{ }^{1} \mathrm{H}$ NMR $(300$ $\left.\mathrm{MHz}, \mathrm{CDCl}_{3}\right) \delta 7.91-7.84(\mathrm{~m}, 2 \mathrm{H}), 7.43-7.38(\mathrm{~m}, 3 \mathrm{H}), 7.38-7.29(\mathrm{~m}, 4 \mathrm{H}), 7.29-7.24(\mathrm{~m}, 2 \mathrm{H}), 7.15$ $-7.04(\mathrm{~m}, 3 \mathrm{H}), 4.41-4.30(\mathrm{~m}, 1 \mathrm{H}), 4.19-3.96(\mathrm{~m}, 4 \mathrm{H}), 3.84-3.73(\mathrm{~m}, 1 \mathrm{H}), 3.72-3.59(\mathrm{~m}, 1 \mathrm{H}), 1.06$ (t, $J=7.1 \mathrm{~Hz}, 3 \mathrm{H}) ;{ }^{13} \mathrm{C} \mathrm{NMR}\left(75 \mathrm{MHz}, \mathrm{CDCl}_{3}\right) \delta 167.2,163.9,158.2,156.5,141.6,137.2,133.8,132.9$, 130.0, 128.8, 128.6, 128.5, 127.3, 127.0, 126.7, 126.1, 125.4, 112.6, 108.7, 60.1, 36.9, 32.5, 29.2, 13.6; IR (film) $v_{\max } 2900,2379,2345,2331,1705,1639,1601,1557,1502,1455,1443,1362,1300,1264$, 1191, 1108, 1069, 1038, 751, 700, 682, 655, 618, $504 \mathrm{~cm}^{-1}$; HRMS (ESI) calcd for $\mathrm{C}_{29} \mathrm{H}_{25} \mathrm{ClNO}_{3} \mathrm{~S}^{+}$ $[\mathrm{M}+\mathrm{H}]^{+}$502.1238, found 502.1247. 

propanoate (3ja)<smiles>CCOC(=O)/C(Cc1cccc(Cl)c1)=C1/CC(c2ccccc2)c2sc(-c3ccccc3)nc2O1</smiles>

Prepared according to the general procedure as described above in $72 \%$ yield $(36.0 \mathrm{mg})$. It was purified by flash chromatography $(5.0 \% \mathrm{EtOAc} / \mathrm{PE})$ to afford an orange solid. $\mathrm{mp}=102-106{ }^{\circ} \mathrm{C} ;{ }^{1} \mathrm{H} \mathrm{NMR}(300$ $\left.\mathrm{MHz} \mathrm{CDCl}_{3}\right) \delta 7.96-7.86(\mathrm{~m}, 2 \mathrm{H}), 7.49-7.39(\mathrm{~m}, 3 \mathrm{H}), 7.39-7.28(\mathrm{~m}, 4 \mathrm{H}), 7.27-7.17(\mathrm{~m}, 5 \mathrm{H}), 4.39$ $-4.30(\mathrm{~m}, 1 \mathrm{H}), 4.14-4.04(\mathrm{~m}, 2 \mathrm{H}), 3.95(\mathrm{q}, J=7.1 \mathrm{~Hz}, 2 \mathrm{H}), 3.83-3.73(\mathrm{~m}, 1 \mathrm{H}), 3.67-3.53(\mathrm{~m}, 1 \mathrm{H})$, $1.17(\mathrm{t}, J=7.1 \mathrm{~Hz}, 3 \mathrm{H}) ;{ }^{13} \mathrm{C} \mathrm{NMR}\left(75 \mathrm{MHz}, \mathrm{CDCl}_{3}\right) \delta 167.2,164.1,158.3,156.5,142.3,141.8,133.7$, $133.1,130.2,129.2,128.8,128.7,128.6,127.4,127.1,126.8,125.9,125.6,113.9,109.0,60.4,37.1,32.7$, 31.5, 13.9; IR (film) $v_{\max } 2980,2378,2345,1705,1635,1598,1557,1503,1455,1430,1362,1301,1260$, $1185,1159,1105,1032,763,700,681 \mathrm{~cm}^{-1}$; HRMS (ESI) calcd for $\mathrm{C}_{29} \mathrm{H}_{25} \mathrm{ClNO}_{3} \mathrm{~S}^{+}[\mathrm{M}+\mathrm{H}]^{+} 502.1238$, found 502.1248 .

\section{Ethyl (E)-3-(4-chlorophenyl)-2-(2,7-diphenyl-6,7-dihydro-5 $H$-pyrano[2,3-d]thiazol-5-ylidene) propanoate (3ka)}<smiles>CCOC(=O)/C(Cc1ccc(Cl)cc1)=C1/CC(c2ccccc2)c2sc(-c3ccccc3)nc2O1</smiles>

Prepared according to the general procedure as described above in $72 \%$ yield $(36.0 \mathrm{mg})$. It was purified by flash chromatography $(5.0 \% \mathrm{EtOAc} / \mathrm{PE})$ to afford a rufous solid. $\mathrm{mp}=75-81{ }^{\circ} \mathrm{C} ;{ }^{1} \mathrm{H} \mathrm{NMR}(300$ $\left.\mathrm{MHz}, \mathrm{CDCl}_{3}\right) \delta 7.94-7.87(\mathrm{~m}, 2 \mathrm{H}), 7.47-7.40(\mathrm{~m}, 3 \mathrm{H}), 7.36-7.28(\mathrm{~m}, 3 \mathrm{H}), 7.25-7.18(\mathrm{~m}, 6 \mathrm{H}), 4.33$ $(\mathrm{dd}, J=7.2,5.6 \mathrm{~Hz}, 1 \mathrm{H}), 4.14-3.86(\mathrm{~m}, 4 \mathrm{H}), 3.81-3.71(\mathrm{~m}, 1 \mathrm{H}), 3.64-3,54(\mathrm{dd}, 1 \mathrm{H}), 1.16(\mathrm{t}, J=7.1$ $\mathrm{Hz}, 3 \mathrm{H}) ;{ }^{13} \mathrm{C} \mathrm{NMR}\left(75 \mathrm{MHz}, \mathrm{CDCl}_{3}\right) \delta 167.1,164.0,157.9,156.4,141.6,138.5,132.9,131.2,130.0$, $129.7,128.6,128.5,127.9,127.3,126.9,125.5,114.0,108.8,60.2,36.9,32.6,31.0,13.7$; IR (film) $v_{\max }$ $2981,2365,2345,1705,1639,1557,1491,1455,1362,1259,1185,1106,1070,1016,804,763,738$, $700 \mathrm{~cm}^{-1}$; HRMS (ESI) calcd for $\mathrm{C}_{29} \mathrm{H}_{25} \mathrm{ClNO}_{3} \mathrm{~S}^{+}[\mathrm{M}+\mathrm{H}]^{+}$502.1238, found 502.1247. 
<smiles>CCOC(=O)/C(Cc1ccccc1Br)=C1/CC(c2ccccc2)c2sc(-c3ccccc3)nc2O1</smiles>

Prepared according to the general procedure as described above in $70 \%$ yield $(38.2 \mathrm{mg})$. It was purified by flash chromatography $(5.0 \% \mathrm{EtOAc} / \mathrm{PE})$ to afford a rufous solid. $\mathrm{mp}=79-86{ }^{\circ} \mathrm{C} ;{ }^{1} \mathrm{H} \mathrm{NMR}(300$ $\left.\mathrm{MHz}, \mathrm{CDCl}_{3}\right) \delta 7.91-7.81(\mathrm{~m}, 2 \mathrm{H}), 7.58-7.51(\mathrm{~m}, 1 \mathrm{H}), 7.43-7.40(\mathrm{~m}, 3 \mathrm{H}), 7.39-7.28(\mathrm{~m}, 4 \mathrm{H}), 7.27$ - $6.99(\mathrm{~m}, 4 \mathrm{H}), 4.42-4.32(\mathrm{~m}, 1 \mathrm{H}), 4.12$ (q, J = 7.1 Hz, 2H), $4.07-3.98(\mathrm{~m}, 2 \mathrm{H}), 3.85-3.75(\mathrm{~m}, 1 \mathrm{H})$, $3.74-65(\mathrm{~m}, 1 \mathrm{H}), 1.07(\mathrm{t}, J=7.1 \mathrm{~Hz}, 3 \mathrm{H}) ;{ }^{13} \mathrm{C} \mathrm{NMR}\left(75 \mathrm{MHz}, \mathrm{CDCl}_{3}\right) \delta 167.3,164.1,158.4,156.6$, $141.8,139.0,133.0,132.3,130.1,128.8,128.7,128.7,127.4,127.2,127.0,125.6,124.5,112.9,108.8$, 60.3, 37.0, 32.7, 32.1, 13.8; IR (film) $v_{\max }$ 2982, 2870, 2393, 2346, 2321, 1705, 1639, 1601, 1557, 1455 , 1427, 1362, 1296, 1263, 1191, 1106, 1068, 1027, 748, 699, 681, 656, $641 \mathrm{~cm}^{-1}$; HRMS (ESI) calcd for $\mathrm{C}_{29} \mathrm{H}_{25} \mathrm{BrNO}_{3} \mathrm{~S}^{+}[\mathrm{M}+\mathrm{H}]^{+}$546.0733, found 546.0744.

Ethyl (E)-3-(3-bromophenyl)-2-(2,7-diphenyl-6,7-dihydro-5H-pyrano[2,3-d]thiazol-5-ylidene) propanoate (3ma)<smiles>CCOC(=O)C(Cc1cccc(Br)c1)=C1CC(c2ccccc2)c2sc(-c3ccccc3)nc2O1</smiles>

Prepared according to the general procedure as described above in $71 \%$ yield (38.8 $\mathrm{mg})$. It was purified by flash chromatography $\left(5.0 \%\right.$ EtOAc/PE) to afford an orange solid. $\mathrm{mp}=110-117{ }^{\circ} \mathrm{C} ;{ }^{1} \mathrm{H} \mathrm{NMR}(300$ $\left.\mathrm{MHz}, \mathrm{CDCl}_{3}\right) \delta 7.96-7.87(\mathrm{~m}, 2 \mathrm{H}), 7.49(\mathrm{~d}, J=1.6 \mathrm{~Hz}, 1 \mathrm{H}), 7.46-7.40(\mathrm{~m}, 3 \mathrm{H}), 7.38-7.30(\mathrm{~m}, 4 \mathrm{H})$, $7.27-7.09(\mathrm{~m}, 4 \mathrm{H}), 4.39-4.29(\mathrm{~m}, 1 \mathrm{H}), 4.16-4.06(\mathrm{~m}, 2 \mathrm{H}), 3.97$ (q, J= $7.1 \mathrm{~Hz}, 2 \mathrm{H}), 3.83-3.74(\mathrm{~m}$, $1 \mathrm{H}), 3.67-3.57(\mathrm{~m}, 1 \mathrm{H}), 1.18(\mathrm{t}, J=7.1 \mathrm{~Hz}, 3 \mathrm{H}) ;{ }^{13} \mathrm{C} \mathrm{NMR}\left(75 \mathrm{MHz}, \mathrm{CDCl}_{3}\right) \delta 167.2,164.1,158.3$, $156.5,142.6,141.8,133.1,131.6,130.2,129.5,128.8,128.8,128.7,127.4,127.3,127.1,125.7,122.0$, 113.9, 109.0, 60.4, 37.1, 32.7, 31.5, 13.9; IR (film) $v_{\max } 2870,2380,2345,1704,1594,1557,1455,1426$, 1361, 1302, 1264, 1182, 1159, 1104, 1069, 1033, 763, 746, 699, 680, $656 \mathrm{~cm}^{-1}$; HRMS (ESI) calcd for $\mathrm{C}_{29} \mathrm{H}_{25} \mathrm{BrNO}_{3} \mathrm{~S}^{+}[\mathrm{M}+\mathrm{H}]^{+}$546.0733, found 546.0745. 
<smiles>CCOC(=O)/C(Cc1ccc(Br)cc1)=C1\CC(c2ccccc2)c2sc(-c3ccccc3)nc2O1</smiles>

Prepared according to the general procedure as described above in $72 \%$ yield (39.3 $\mathrm{mg}$ ). It was purified by flash chromatography $(5.0 \% \mathrm{EtOAc} / \mathrm{PE})$ to afford a rufous solid. $\mathrm{mp}=73-81{ }^{\circ} \mathrm{C} ;{ }^{1} \mathrm{H} \mathrm{NMR}(300$ $\left.\mathrm{MHz}, \mathrm{CDCl}_{3}\right) \delta 7.97-7.87(\mathrm{~m}, 2 \mathrm{H}), 7.60-7.50(\mathrm{~m}, 2 \mathrm{H}), 7.50-7.39(\mathrm{~m}, 5 \mathrm{H}), 7.38-7.28(\mathrm{~m}, 3 \mathrm{H}), 7.27$ $-7.20(\mathrm{~m}, 2 \mathrm{H}), 4.39-4.31(\mathrm{~m}, 1 \mathrm{H}), 4.16-3.98(\mathrm{~m}, 4 \mathrm{H}), 3.85-3.75(\mathrm{~m}, 1 \mathrm{H}), 3.71-3.61(\mathrm{~m}, 1 \mathrm{H}), 1.17$ $(\mathrm{t}, J=7.1 \mathrm{~Hz}, 3 \mathrm{H}) ;{ }^{13} \mathrm{C} \mathrm{NMR}\left(75 \mathrm{MHz}, \mathrm{CDCl}_{3}\right) \delta 167.1,164.0,158.0,156.4,141.6,139.1,132.9,130.8$, 130.1, 130.0, 128.6, 128.5, 127.3, 127.0, 125.5, 119.3, 113.9, 108.8, 60.2, 36.9, 32.6, 31.0, 13.8; IR (film) $v_{\max } 2983,2366,2345,1706,1639,1557,1502,1455,1420,1362,1326,1278,1257,1162,1109,1067$, 1019, 951, 860, 819, 763, 739, $700 \mathrm{~cm}^{-1}$; HRMS (ESI) calcd for $\mathrm{C}_{29} \mathrm{H}_{25} \mathrm{BrNO}_{3} \mathrm{~S}^{+}[\mathrm{M}+\mathrm{H}]^{+}$546.0733, found 546.0744.

Ethyl (E)-2-(2,7-diphenyl-6,7-dihydro-5H-pyrano[2,3-d]thiazol-5-ylidene)-3-(3(trifluoromethyl) phenyl)propanoate (3oa)<smiles>CCOC(=O)C(Cc1cccc(C(F)(F)F)c1)=C1CC(c2ccccc2)c2sc(-c3ccccc3)nc2O1</smiles>

Prepared according to the general procedure as described above in $70 \%$ yield $(37.5 \mathrm{mg})$. It was purified by flash chromatography $\left(5.0 \%\right.$ EtOAc/PE) to afford an orange solid. $\mathrm{mp}=118-126{ }^{\circ} \mathrm{C} ;{ }^{1} \mathrm{H} \mathrm{NMR}(300$ $\left.\mathrm{MHz}, \mathrm{CDCl}_{3}\right) \delta 7.96-7.87(\mathrm{~m}, 2 \mathrm{H}), 7.61(\mathrm{~s}, 1 \mathrm{H}), 7.53-7.41(\mathrm{~m}, 5 \mathrm{H}), 7.40-7.28(\mathrm{~m}, 4 \mathrm{H}), 7.27-7.18$ (m, 2H), $4.38-4.31(\mathrm{~m}, 1 \mathrm{H}), 4.11(\mathrm{q}, J=7.1 \mathrm{~Hz}, 2 \mathrm{H}), 4.07-3.98(\mathrm{~m}, 2 \mathrm{H}), 3.84-3.75(\mathrm{~m}, 1 \mathrm{H}), 3.66-$ $3.57(\mathrm{~m}, 1 \mathrm{H}), 1.17(\mathrm{t}, J=7.1 \mathrm{~Hz}, 3 \mathrm{H}) ;{ }^{13} \mathrm{C} \mathrm{NMR}\left(75 \mathrm{MHz}, \mathrm{CDCl}_{3}\right) \delta 167.1,164.2,158.5,156.5,141.8$, $141.2,133.1,132.0,130.20$ (q, $J=31.9 \mathrm{~Hz}), 130.17,128.8,128.6,128.4,127.4,127.1,125.6,125.4(\mathrm{q}$, $J=3.78 \mathrm{~Hz}$ ), 122.6 (q, $J=3.78 \mathrm{~Hz}), 113.8,109.0,60.4,37.1,32.7,31.6,13.8$; IR (film) $v_{\max } 2982,2345$, $1706,1637,1557,1494,1452,1362,1332,1276,1257,1164,1122,1074,1034,964,763,745,701,658$ $\mathrm{cm}^{-1}$; HRMS (ESI) calcd for $\mathrm{C}_{30} \mathrm{H}_{25} \mathrm{~F}_{3} \mathrm{NO}_{3} \mathrm{~S}^{+}[\mathrm{M}+\mathrm{H}]^{+}$536.1502, found 536.1507. 
Ethyl (E)-2-(2,7-diphenyl-6,7-dihydro-5H-pyrano[2,3-d]thiazol-5-ylidene)-3-(4(trifluoromethyl) phenyl)propanoate (3pa)<smiles>CCOC(=O)/C(Cc1ccc(C(F)(F)F)cc1)=C1/CC(c2ccccc2)c2sc(-c3ccccc3)nc2O1</smiles>

Prepared according to the general procedure as described above in $70 \%$ yield $(37.5 \mathrm{mg})$. It was purified by flash chromatography $(5.0 \% \mathrm{EtOAc} / \mathrm{PE})$ to afford a rufous solid. $\mathrm{mp}=56-61{ }^{\circ} \mathrm{C} ;{ }^{1} \mathrm{H} \mathrm{NMR}(300$ $\left.\mathrm{MHz}, \mathrm{CDCl}_{3}\right) \delta 7.95-7.87(\mathrm{~m}, 2 \mathrm{H}), 7.55-7.48(\mathrm{~m}, 2 \mathrm{H}), 7.48-7.37(\mathrm{~m}, 5 \mathrm{H}), 7.37-7.29(\mathrm{~m}, 3 \mathrm{H}), 7.26$ $-7.18(\mathrm{~m}, 2 \mathrm{H}), 4.41-4.31(\mathrm{~m}, 1 \mathrm{H}), 4.17-3.94(\mathrm{~m}, 4 \mathrm{H}), 3.84-3.74(\mathrm{~m}, 1 \mathrm{H}), 3.69-3.58(\mathrm{~m}, 1 \mathrm{H}), 1.16$ $(\mathrm{t}, J=7.1 \mathrm{~Hz}, 3 \mathrm{H}) ;{ }^{13} \mathrm{C} \mathrm{NMR}\left(75 \mathrm{MHz}, \mathrm{CDCl}_{3}\right) \delta 167.2,164.2,158.5,156.5,144.4,141.7,133.0,130.2$, 128.8, 128.7, 128.6, 127.4, 127.1, 125.6, 124.9 (q, $J=3.7 \mathrm{~Hz}), 113.6,109.0,60.4,37.0,32.7,31.6,13.8$; IR (film) $v_{\max } 2982,2345,1706,1640,1557,1502,1455,1419,1362,1326,1278,1258,1163,1109$, 1067, 1020, 951, 763, 740, $700 \mathrm{~cm}^{-1}$; HRMS (ESI) calcd for $\mathrm{C}_{30} \mathrm{H}_{25} \mathrm{~F}_{3} \mathrm{NO}_{3} \mathrm{~S}^{+}[\mathrm{M}+\mathrm{H}]^{+}$536.1502, found 536.1509.

\section{Methyl (E)-4-(2-(2,7-diphenyl-6,7-dihydro-5H-pyrano[2,3-d]thiazol-5-ylidene)-3-ethoxy-3-} oxopropyl)benzoate (3qa)<smiles>CCOC(=O)/C(Cc1ccc(C(C)=O)cc1)=C1/CC(c2ccccc2)c2sc(-c3ccccc3)nc2O1</smiles>

Prepared according to the general procedure as described above in $69 \%$ yield (36.3 $\mathrm{mg})$. It was purified by flash chromatography $\left(5.0 \%\right.$ EtOAc/PE) to afford an orange solid. $\mathrm{mp}=122-126{ }^{\circ} \mathrm{C} ;{ }^{1} \mathrm{H} \mathrm{NMR}(300$ $\left.\mathrm{MHz} \mathrm{CDCl}_{3}\right) \delta 7.98-7.84(\mathrm{~m}, 4 \mathrm{H}), 7.45-7.38(\mathrm{~m}, 3 \mathrm{H}), 7.38-7.28(\mathrm{~m}, 5 \mathrm{H}), 7.25-7.18(\mathrm{~m}, 2 \mathrm{H}), 4.37$ $-4.29(\mathrm{~m}, 1 \mathrm{H}), 4.13-3.92(\mathrm{~m}, 4 \mathrm{H}), 3.89(\mathrm{~s}, 3 \mathrm{H}), 3.82-3.72(\mathrm{~m}, 1 \mathrm{H}), 3.68-3.58(\mathrm{~m}, 1 \mathrm{H}), 1.13(\mathrm{t}, J=$ $7.1 \mathrm{~Hz}, 3 \mathrm{H}) ;{ }^{13} \mathrm{C} \mathrm{NMR}\left(75 \mathrm{MHz}, \mathrm{CDCl}_{3}\right) \delta 167.2,167.0,164.1,158.4,156.5,145.8,141.7,133.0,130.2$, $129.4,128.8,128.6,128.4,127.7,127.4,127.1,125.6,113.6,109.0,60.3,51.7,37.0,32.7,31.8,13.9$; IR (film) $v_{\max } 2982,2951,1719,1639,1610,1557,1501,1455,1435,1361,1281,1180,1106,1069$, $1021,965,865,809,763,701 \mathrm{~cm}^{-1}$; HRMS (ESI) calcd for $\mathrm{C}_{31} \mathrm{H}_{28} \mathrm{NO}_{5} \mathrm{~S}^{+}[\mathrm{M}+\mathrm{H}]^{+} 526.1683$, found 526.1697. 
Ethyl (E)-2-(2,7-diphenyl-6,7-dihydro-5H-pyrano[2,3-d]thiazol-5-ylidene)-3-(naphthalen-2-

yl) propanoate (3ra)<smiles>CCOC(=O)/C(Cc1ccc2ccccc2c1)=C1/CC(c2ccccc2)c2sc(-c3ccccc3)nc2O1</smiles>

Prepared according to the general procedure as described above in $63 \%$ yield $(32.6 \mathrm{mg})$. It was purified by flash chromatography $\left(5.0 \%\right.$ EtOAc/PE) to afford an orange solid. $\mathrm{mp}=118-124{ }^{\circ} \mathrm{C} ;{ }^{1} \mathrm{H}$ NMR $(300$ $\left.\mathrm{MHz}, \mathrm{CDCl}_{3}\right) \delta 7.95-7.87(\mathrm{~m}, 2 \mathrm{H}), 7.83-7.69(\mathrm{~m}, 4 \mathrm{H}), 7.49-7.37$ (m, 6H), $7.35-7.27(\mathrm{~m}, 3 \mathrm{H}), 7.23$ (dt, $J=7.0,2.4 \mathrm{~Hz}, 2 \mathrm{H}), 4.34(\mathrm{dd}, J=7.4,5.6 \mathrm{~Hz}, 1 \mathrm{H}), 4.25-4.01(\mathrm{~m}, 4 \mathrm{H}), 3.81(\mathrm{dd}, J=14.9,5.6 \mathrm{~Hz}$, $1 \mathrm{H}), 3.65-3.54(\mathrm{~m}, 1 \mathrm{H}), 1.14(\mathrm{t}, J=7.1 \mathrm{~Hz}, 3 \mathrm{H}) ;{ }^{13} \mathrm{C} \mathrm{NMR}\left(75 \mathrm{MHz}, \mathrm{CDCl}_{3}\right) \delta$ 167.4, 163.9, 157.6, 156.6, 141.8, 137.5, 133.3, 133.0, 131.8, 130.0, 128.6, 128.5, 127.32, 127.25, 127.2, 127.0, 126.5, 125.5, 125.3, 124.7, 114.4, 108.8, 60.2, 37.1, 32.6, 31.8, 13.7; IR (film) $v_{\max } 3057,2979,1704,1636,1601$, $1555,1501,1454,1361,1284,1258,1184,1102,1069,1031,950,860,795,762,700,474 \mathrm{~cm}^{-1}$; HRMS (ESI) calcd for $\mathrm{C}_{33} \mathrm{H}_{28} \mathrm{NO}_{3} \mathrm{~S}^{+}[\mathrm{M}+\mathrm{H}]^{+} 518.1784$, found 518.1788 .

\section{Characterization Data for the Chiral Products (+)-3}

(+)-Ethyl

(E)-2-(2,7-diphenyl-6,7-dihydro-5H-pyrano[2,3-d]thiazol-5-ylidene)-3-phenyl propanoate ((+)-3aa)<smiles>CCOC(=O)C(Cc1ccccc1)=C1CC(c2ccccc2)c2sc(-c3ccccc3)nc2O1</smiles>

Prepared according to the general procedure as described above in $80 \%$ yield $(18.8 \mathrm{mg})$. It was purified by flash chromatography $(5.0 \% \mathrm{EtOAc} / \mathrm{PE})$ to afford a rufous solid. $\mathrm{mp}=82-90{ }^{\circ} \mathrm{C} ;[\alpha]^{25} \mathrm{D}=+7.2(\mathrm{c}$ 1.16, $\left.\mathrm{CH}_{2} \mathrm{Cl}_{2}\right) ;{ }^{1} \mathrm{H}$ NMR $\left(300 \mathrm{MHz}, \mathrm{CDCl}_{3}\right) \delta 7.96-7.86(\mathrm{~m}, 2 \mathrm{H}), 7.48-7.40(\mathrm{~m}, 3 \mathrm{H}), 7.35-7.29(\mathrm{~m}$, 5H), $7.28-7.15$ (m, 5H), $4.38-4.28$ (q, J= $7.1 \mathrm{~Hz}, 1 \mathrm{H}), 4.14-4.04$ (m, 2H), 3.97 (q, J= 7.1 Hz, 2H), $3.86-3.76(\mathrm{~m}, 1 \mathrm{H}), 3.61-3.51(\mathrm{~m}, 1 \mathrm{H}), 1.16(\mathrm{t}, J=7.1 \mathrm{~Hz}, 3 \mathrm{H}) ;{ }^{13} \mathrm{C} \mathrm{NMR}\left(75 \mathrm{MHz}, \mathrm{CDCl}_{3}\right) \delta$ 167.6, 164.0 , 157.6 , 156.7 , 141.9, 140.1, 133.1, 130.1, 128.8,128.6, 128.5, 128.0, 127.4, 127.2 , 125.7, 125.5 , 114.6, 109.0, 60.3, 37.2, 32.7, 31.7, 13.9; IR (film) $v_{\max } 3029$, 2981, 2378, 2345, 1704, 1632, 1601, 1556, $1495,1454,1361,1275,1186,1155,1104,1032,763,700 \mathrm{~cm}^{-1}$; HRMS (ESI) calcd for $\mathrm{C}_{29} \mathrm{H}_{26} \mathrm{NO}_{3} \mathrm{~S}^{+}$ $[\mathrm{M}+\mathrm{H}]^{+} 468.1628$, found 468.1638. HPLC analysis: 98\% ee (R\&C RC-OD, 10\% isopropanol/hexane, $1.0 \mathrm{~mL} / \mathrm{min}, \mathrm{UV}: 254 \mathrm{~nm}$ ), $t_{R}=6.2 \mathrm{~min}$ (minor), $10.1 \mathrm{~min}$ (major). 
(+)-Ethyl (E)-2-(7-(4-bromophenyl)-2-phenyl-6,7-dihydro-5H-pyrano[2,3-d]thiazol-5ylidene)-3-phenylpropanoate ((+)-3aj)<smiles>CCOC(=O)/C(Cc1ccccc1)=C1/CC(c2ccc(Br)cc2)c2sc(-c3ccccc3)nc2O1</smiles>

Prepared according to the general procedure as described above in $86 \%$ yield $(23.4 \mathrm{mg})$. It was purified by flash chromatography $(5.0 \% \mathrm{EtOAc} / \mathrm{PE})$ to afford a rufous solid. $\mathrm{mp}=57-65^{\circ} \mathrm{C} ;[\alpha]^{25} \mathrm{D}=+17.8(\mathrm{c}$ 1.16, $\left.\mathrm{CH}_{2} \mathrm{Cl}_{2}\right) ;{ }^{1} \mathrm{H}$ NMR $\left(300 \mathrm{MHz}, \mathrm{CDCl}_{3}\right) \delta 7.95-7.88(\mathrm{~m}, 2 \mathrm{H}), 7.47-7.40(\mathrm{~m}, 5 \mathrm{H}), 7.31-7.23(\mathrm{~m}$, 4H), $7.21-7.14(\mathrm{~m}, 1 \mathrm{H}), 7.11-7.05(\mathrm{~m}, 2 \mathrm{H}), 4.35-4.26(\mathrm{~m}, 1 \mathrm{H}), 4.15-3.91(\mathrm{~m}, 4 \mathrm{H}), 3.74-3.56(\mathrm{~m}$, 2H), $1.16(\mathrm{t}, J=7.1 \mathrm{~Hz}, 3 \mathrm{H}) ;{ }^{13} \mathrm{C} \mathrm{NMR}\left(75 \mathrm{MHz}, \mathrm{CDCl}_{3}\right) \delta 167.3,164.1,156.9,156.6,140.8,139.8$, 132.8, 131.6, 130.1, 128.7, 128.6, 128.3, 127.8, 125.5, 125.5, 121.1, 115.0, 107.78, 60.2, 36.5, 32.3, 31.5, 13.7; IR (film) $v_{\max } 3028,2980,1704,1640,1556,1489,1454,1407,1361,1274,1182,1105,1074$, $1031,1011,953,880,822,762,737,700 \mathrm{~cm}^{-1}$; HRMS (ESI) calcd for $\mathrm{C}_{29} \mathrm{H}_{25} \mathrm{BrNO}_{3} \mathrm{~S}^{+}[\mathrm{M}+\mathrm{H}]^{+} 546.0733$, found 546.0743. HPLC analysis: 95\% ee (R\&C RC-OD, 10\% isopropanol/hexane, $1.0 \mathrm{~mL} / \mathrm{min}$, UV: 254 $\mathrm{nm}$ ), $t_{R}=7.8 \mathrm{~min}$ (minor), $12.4 \mathrm{~min}$ (major).

(+)-Ethyl (E)-3-(3-bromophenyl)-2-(2,7-diphenyl-6,7-dihydro-5H-pyrano[2,3-d]thiazol-5-ylidene) Propanoate ((+)-3ma)<smiles></smiles>

Prepared according to the general procedure as described above in $75 \%$ yield $(20.4 \mathrm{mg})$. It was purified by flash chromatography $\left(5.0 \%\right.$ EtOAc/PE) to afford an orange solid. $\mathrm{mp}=110-117^{\circ} \mathrm{C} ;[\alpha]^{25} \mathrm{D}=+21.6$ (c 1.16, $\left.\mathrm{CH}_{2} \mathrm{Cl}_{2}\right) ;{ }^{1} \mathrm{H}$ NMR $\left(300 \mathrm{MHz}, \mathrm{CDCl}_{3}\right) \delta 7.96-7.87(\mathrm{~m}, 2 \mathrm{H}), 7.49(\mathrm{~d}, J=1.6 \mathrm{~Hz}, 1 \mathrm{H}), 7.46-$ $7.40(\mathrm{~m}, 3 \mathrm{H}), 7.38-7.30(\mathrm{~m}, 4 \mathrm{H}), 7.27-7.09(\mathrm{~m}, 4 \mathrm{H}), 4.39-4.29(\mathrm{~m}, 1 \mathrm{H}), 4.16-4.06(\mathrm{~m}, 2 \mathrm{H}), 3.97$ $(\mathrm{q}, J=7.1 \mathrm{~Hz}, 2 \mathrm{H}), 3.83-3.74(\mathrm{~m}, 1 \mathrm{H}), 3.67-3.57(\mathrm{~m}, 1 \mathrm{H}), 1.18(\mathrm{t}, J=7.1 \mathrm{~Hz}, 3 \mathrm{H}) ;{ }^{13} \mathrm{C}$ NMR $(75$ $\left.\mathrm{MHz}, \mathrm{CDCl}_{3}\right) \delta$ 167.2, 164.1, 158.3, 156.5, 142.6, 141.8, 133.1, 131.6, 130.2, 129.5, 128.8, 128.8, 128.7, 127.4, 127.3, 127.1, 125.7, 122.0, 113.9, 109.0, 60.4, 37.1, 32.7, 31.5, 13.9; IR (film) $v_{\max } 2870,2380$, 2345, 1704, 1594, 1557, 1455, 1426, 1361, 1302, 1264, 1182, 1159, 1104, 1069, 1033, 763, 746, 699, 680, $656 \mathrm{~cm}^{-1}$; HRMS (ESI) calcd for $\mathrm{C}_{29} \mathrm{H}_{25} \mathrm{BrNO}_{3} \mathrm{~S}^{+}[\mathrm{M}+\mathrm{H}]^{+}$546.0733, found 546.0745. HPLC analysis: $99 \%$ ee (R\&C RC-OD, 10\% isopropanol/hexane, $1.0 \mathrm{~mL} / \mathrm{min}, \mathrm{UV}: 254 \mathrm{~nm}$ ), $t_{R}=6.9 \mathrm{~min}$ (minor), 13.1 min (major). 
(+)-Ethyl (E)-3-(4-bromophenyl)-2-(2,7-diphenyl-6,7-dihydro-5H-pyrano[2,3-d]thiazol-5ylidene) Propanoate ((+)-3na)<smiles>CCOC(=O)/C(Cc1ccc(Br)cc1)=C1/CC(c2ccccc2)c2sc(-c3ccccc3)nc2O1</smiles>

Prepared according to the general procedure as described above in $82 \%$ yield $(22.3 \mathrm{mg})$. It was purified by flash chromatography $(5.0 \% \mathrm{EtOAc} / \mathrm{PE})$ to afford a rufous solid. $\mathrm{mp}=73-81^{\circ} \mathrm{C} ;[\alpha]^{25} \mathrm{D}=+22.6(\mathrm{c}$ 1.16, $\left.\mathrm{CH}_{2} \mathrm{Cl}_{2}\right) ;{ }^{1} \mathrm{H}$ NMR $\left(300 \mathrm{MHz}, \mathrm{CDCl}_{3}\right) \delta 7.97-7.87(\mathrm{~m}, 2 \mathrm{H}), 7.60-7.50(\mathrm{~m}, 2 \mathrm{H}), 7.50-7.39(\mathrm{~m}$, $5 \mathrm{H}), 7.38-7.28(\mathrm{~m}, 3 \mathrm{H}), 7.27-7.20(\mathrm{~m}, 2 \mathrm{H}), 4.39-4.31(\mathrm{~m}, 1 \mathrm{H}), 4.16-3.98(\mathrm{~m}, 4 \mathrm{H}), 3.85-3.75(\mathrm{~m}$, 1H), $3.71-3.61(\mathrm{~m}, 1 \mathrm{H}), 1.17(\mathrm{t}, J=7.1 \mathrm{~Hz}, 3 \mathrm{H}) ;{ }^{13} \mathrm{C} \mathrm{NMR}\left(75 \mathrm{MHz}, \mathrm{CDCl}_{3}\right) \delta 167.1,164.0,158.0$, 156.4, 141.6, 139.1, 132.9, 130.8, 130.1, 130.0, 128.6, 128.5, 127.3, 127.0, 125.5, 119.3, 113.9, 108.8, 60.2, 36.9, 32.6, 31.0, 13.8; IR (film) $v_{\max } 2983,2366,2345,1706,1639,1557,1502,1455,1420,1362$, 1326, 1278, 1257, 1162, 1109, 1067, 1019, 951, 860, 819, 763, 739, $700 \mathrm{~cm}^{-1}$; HRMS (ESI) calcd for $\mathrm{C}_{29} \mathrm{H}_{25} \mathrm{BrNO}_{3} \mathrm{~S}^{+}[\mathrm{M}+\mathrm{H}]^{+}$546.0733, found 546.0744. HPLC analysis: 97\% ee (R\&C RC-OD, $10 \%$ isopropanol/hexane, $1.0 \mathrm{~mL} / \mathrm{min}, \mathrm{UV}: 254 \mathrm{~nm}$ ), $t_{R}=6.5 \mathrm{~min}$ (minor), $11.8 \mathrm{~min}$ (major).

\section{Transformations of Product 3aa}<smiles>CCOC(=O)C(Cc1ccccc1)=C1CC(c2ccccc2)c2sc(-c3ccccc3)nc2O1</smiles>

3aa

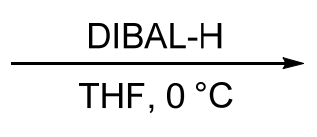

Under $\mathrm{N}_{2}$ atmosphere, $1 \mathrm{~mL}$ of DIBAL-H (1M in Hexane, 10.0 equiv) was dissolved in $1 \mathrm{~mL}$ of THF, then solution of the cycloaddition product 3aa $(46.8 \mathrm{mg}, 0.1 \mathrm{mmol})$ was dissolved in $1 \mathrm{~mL}$ of THF was added dropwise. The resulting mixture was stirred at $0{ }^{\circ} \mathrm{C}$ for $10 \mathrm{~min}$. The residue was purified through flash column chromatography ( $30.0 \% \mathrm{EtOAc} / \mathrm{PE})$ to afford the corresponding product $\mathbf{4}$ as a rufous solid (32.0 mg, $75 \%$ yield). $\mathrm{mp}=67-71^{\circ} \mathrm{C} ;{ }^{1} \mathrm{H}$ NMR $\left(300 \mathrm{MHz}, \mathrm{CDCl}_{3}\right) \delta 7.97-7.87(\mathrm{~m}, 2 \mathrm{H}), 7.45-7.40$ (m, 3H), $7.35-7.27$ (m, 5H), $7.26-7.16(\mathrm{~m}, 5 \mathrm{H}), 4.45-4.25$ (m, 1H), $3.98-3.54$ (m, 4H), 3.10 (dd, $J$ $=14.0,5.5 \mathrm{~Hz}, 1 \mathrm{H}), 2.96(\mathrm{dd}, J=14.1,5.4 \mathrm{~Hz}, 1 \mathrm{H}), 1.27(\mathrm{~s}, 1 \mathrm{H}) ;{ }^{13} \mathrm{C} \mathrm{NMR}\left(75 \mathrm{MHz}, \mathrm{CDCl}_{3}\right) \delta 163.9$, 158.2, 145.0, 142.6, 139.6, 133.1, 129.9, 128.7, 128.6, 128.4, 128.1, 127.2, 127.1, 127.0, 125.7, 125.5, 120.4, 106.4, 60.3, 37.8, 33.0, 31.9; IR (film) $v_{\max } 3062$, 3029, 2922, 1717, 1682, 1601, 1553, 1495, 1454 , 1366, 1264, 1180, 1074, 1030, 763, 737, $700 \mathrm{~cm}^{-1}$; HRMS (ESI) calcd for $\mathrm{C}_{27} \mathrm{H}_{24} \mathrm{NO}_{2} \mathrm{~S}^{+}[\mathrm{M}+\mathrm{H}]^{+}$ 426.1483, found 426.1521 . 


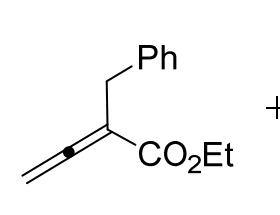

$1 \mathrm{a}$<smiles>O=C1N=C(Pc2ccccc2)SC1=CP</smiles>

$2 a$

$6 \mathrm{mmol}, 1.22 \mathrm{~g} \quad 4 \mathrm{mmol}, 1.06 \mathrm{~g}$

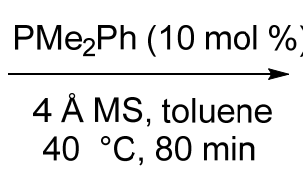

$40{ }^{\circ} \mathrm{C}, 80 \mathrm{~min}$

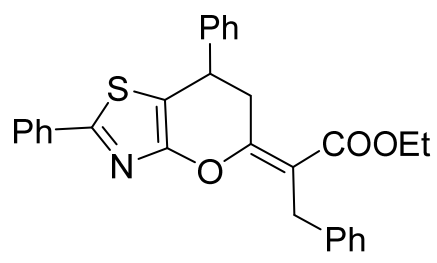

3aa

$1.28 \mathrm{~g}, 68 \%$ yield

Under nitrogen atmosphere, thiazolone-derived alkene $2 \mathbf{a}(1.06 \mathrm{~g}, 4.0 \mathrm{mmol})$ and the additive $4 \AA \mathrm{MS}$ (4.0 g) in $40.0 \mathrm{~mL}$ of toluene, $\alpha$-benzyl allenotes $1 \mathrm{a}(1.22 \mathrm{~g}, 6.0 \mathrm{mmol})$ followed with catalyst $\mathrm{PMe}_{2} \mathrm{Ph}$ (55.2 $\mathrm{mg}, 0.40 \mathrm{mmol}$ ) were additive in a $100 \mathrm{~mL}-$ Shrek tube at $40{ }^{\circ} \mathrm{C}$. The mixture was stirred until starting material was completely consumed (monitored by TLC) and then was concentrated to dryness. The residue was purified through flash column chromatography (5.0\% EtOAc/hexane) to afford the orange product 3aa $(1.28 \mathrm{~g}, 68 \%$ yield $)$. 


\section{Copies of ${ }^{1} \mathrm{H}$ and ${ }^{13} \mathrm{C}$ NMR Spectra}

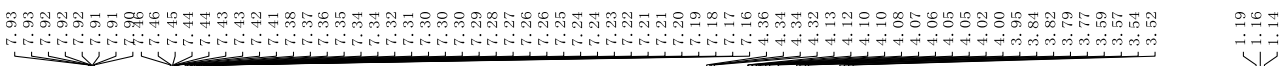<smiles>CCOC(=O)C(Cc1ccccc1)=C1CC(c2ccccc2)c2sc(-c3ccccc3)nc2O1</smiles>

3aa

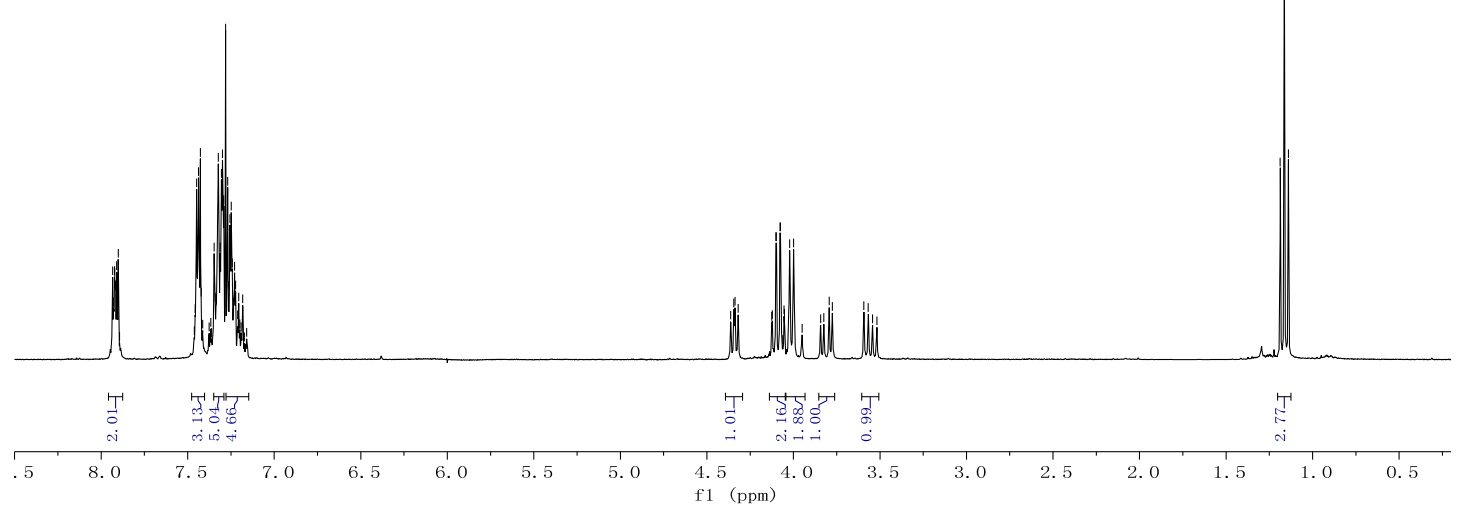

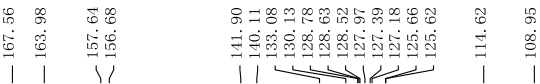

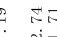

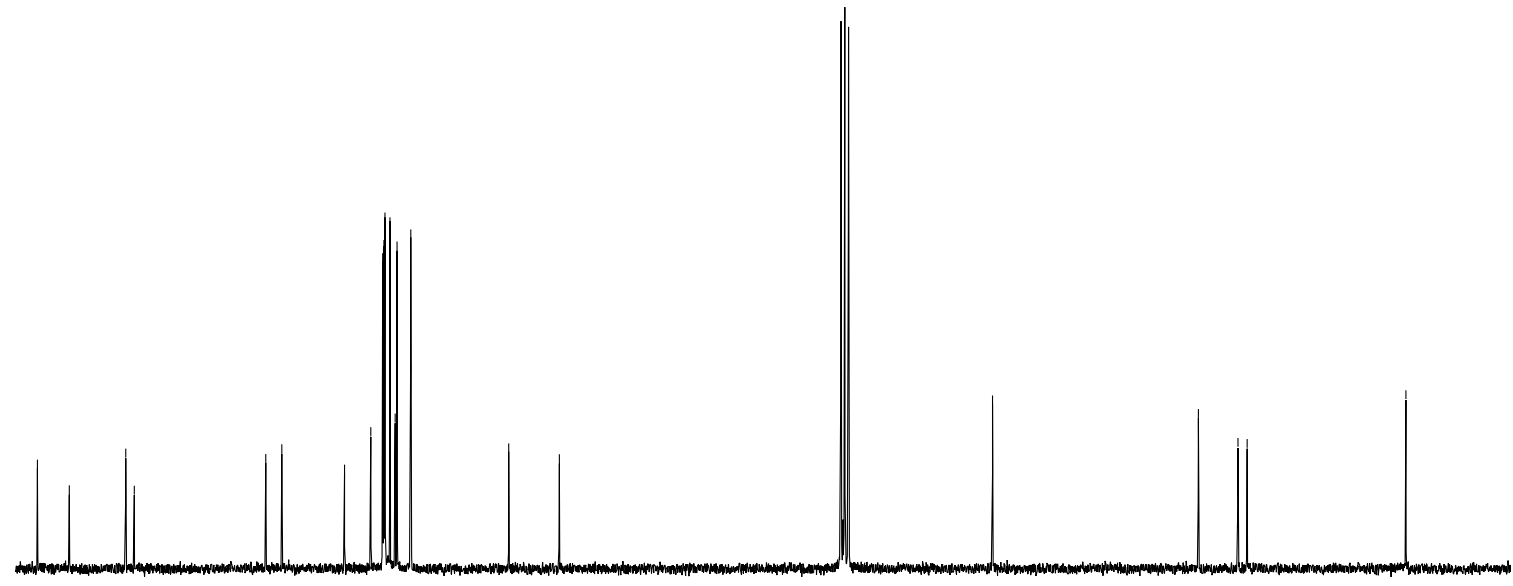

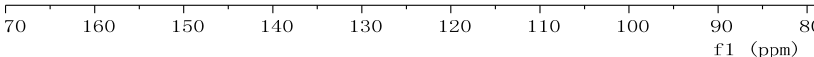


<smiles>CCOC(=O)/C(Cc1ccccc1)=C1/CC(c2ccccc2C)c2sc(-c3ccccc3)nc2O1</smiles>

$3 a b$

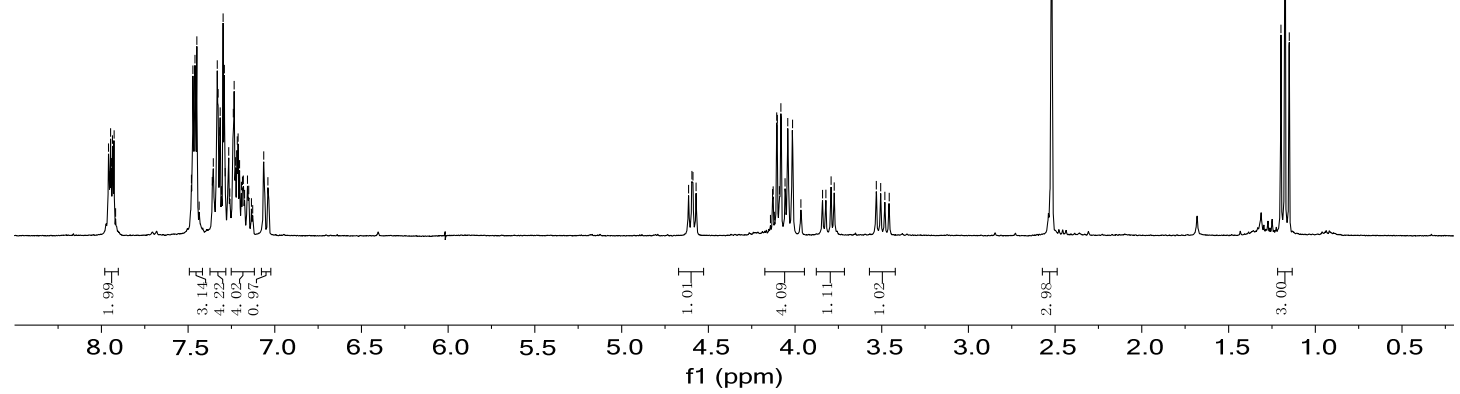

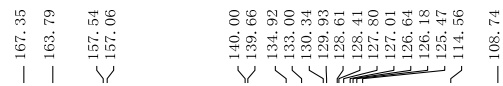

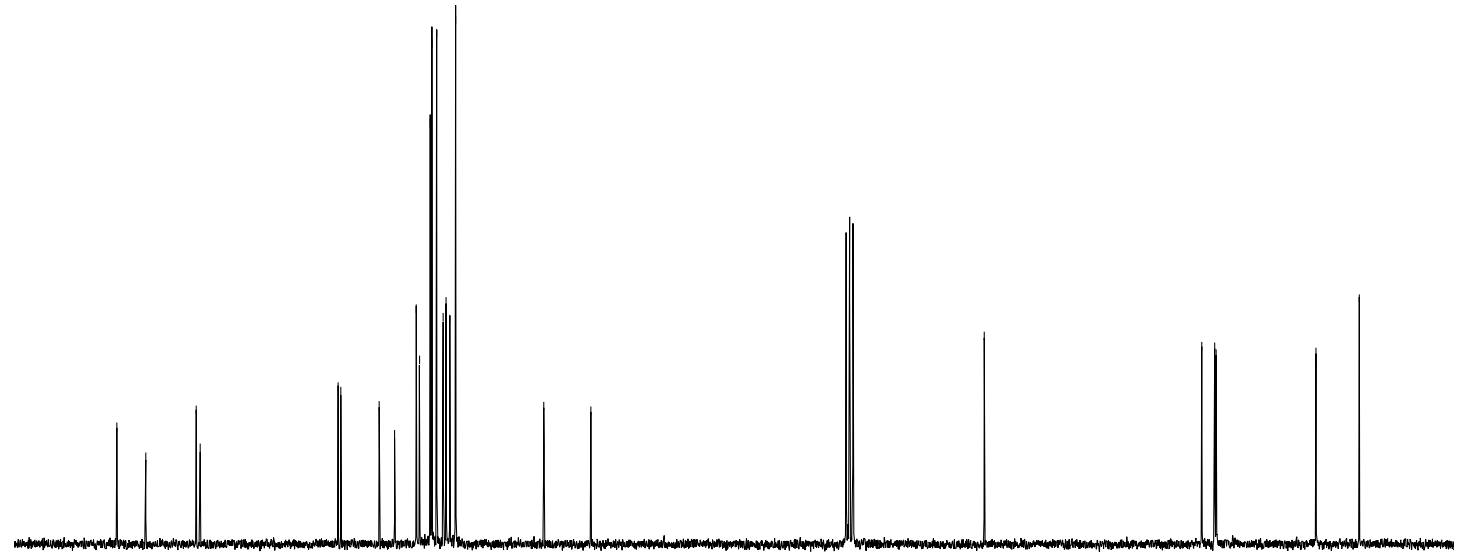

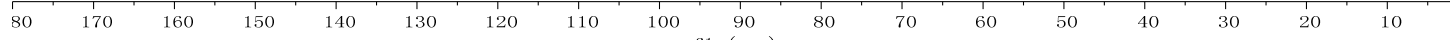


<smiles>CCOC(=O)/C(Cc1ccccc1)=C1/CC(c2cccc(C)c2)c2sc(-c3ccccc3)nc2O1</smiles>

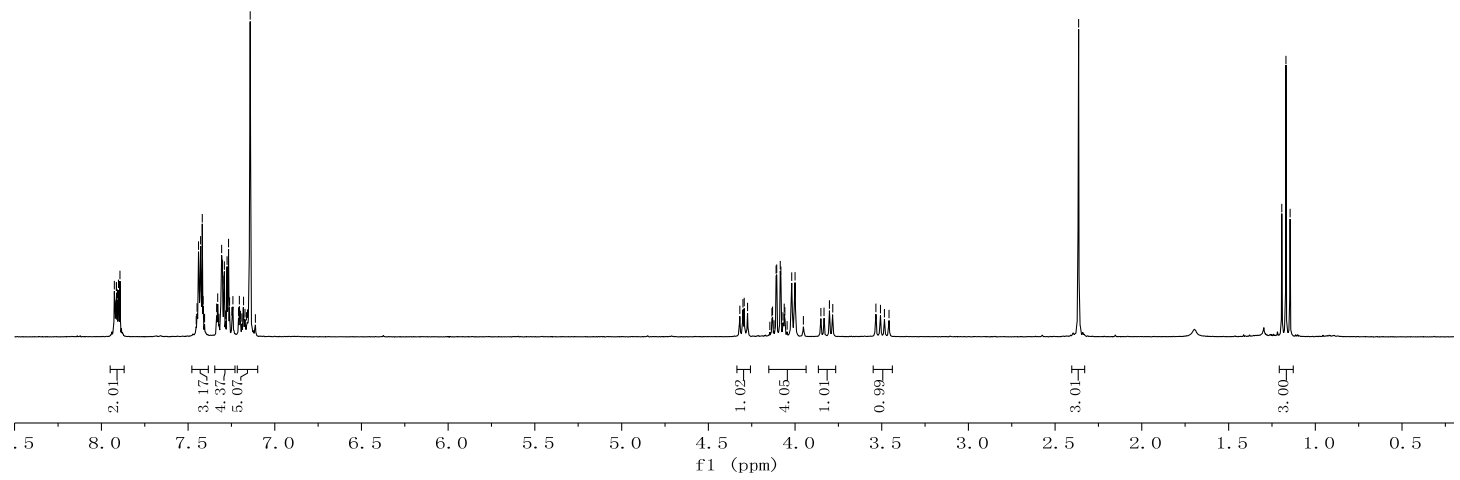

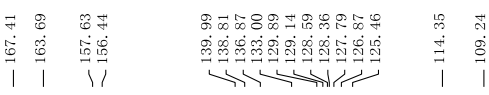

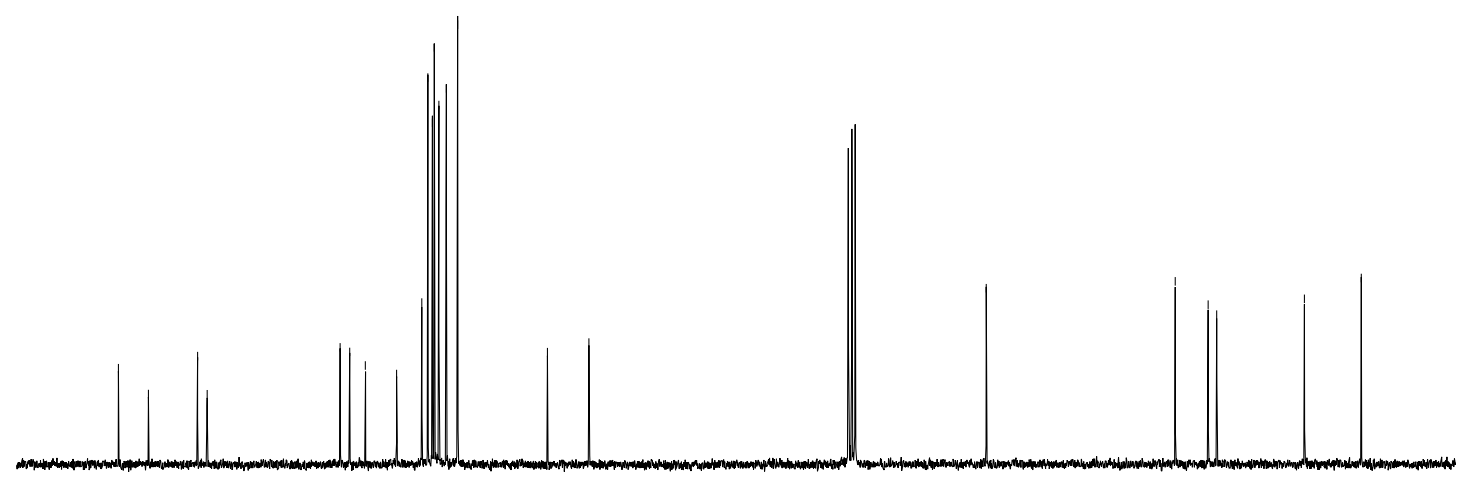

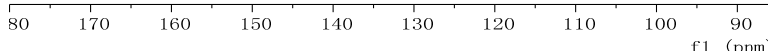




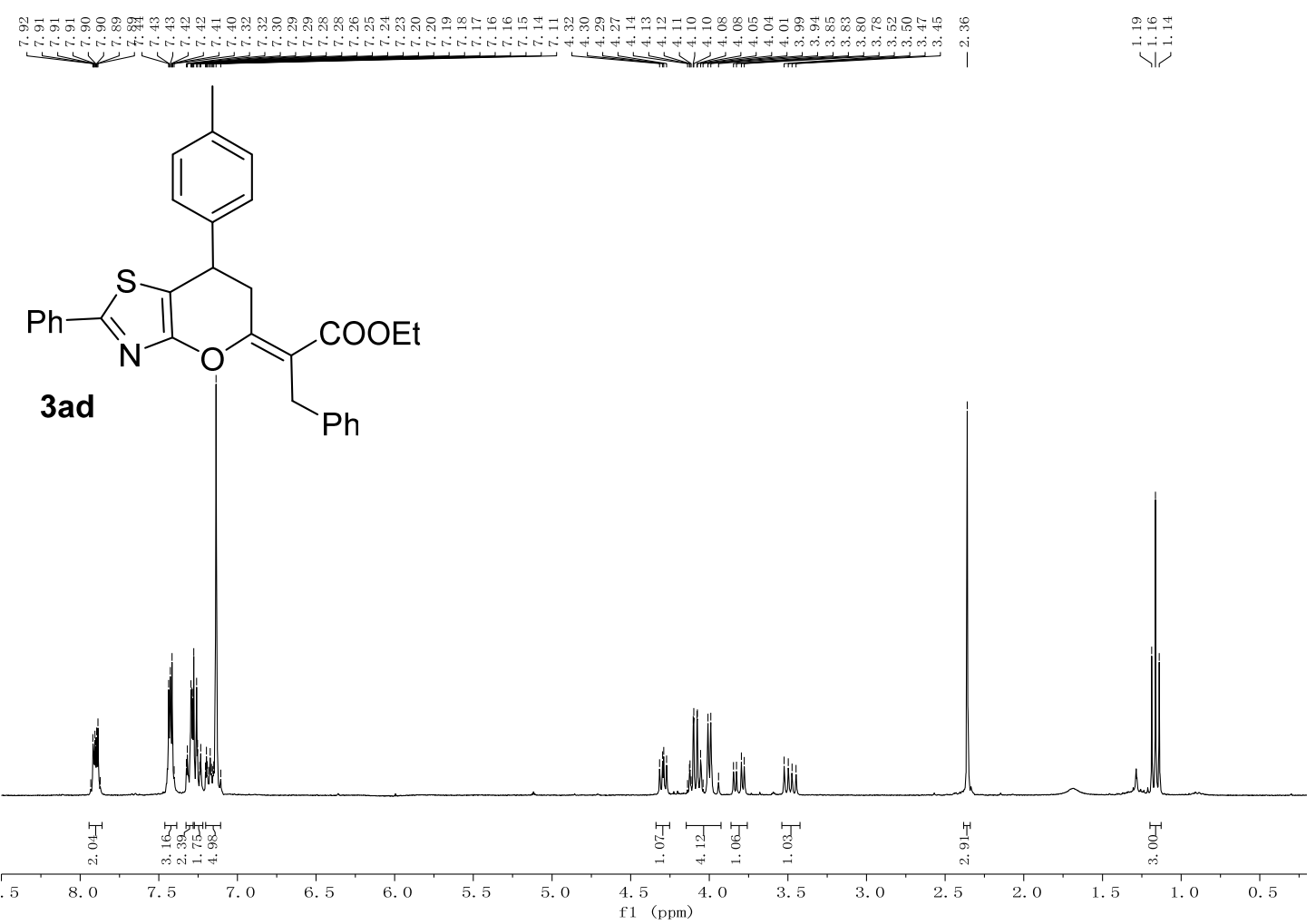

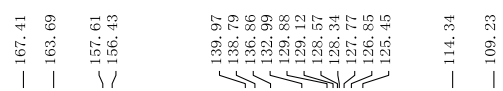

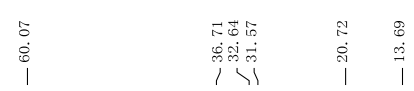

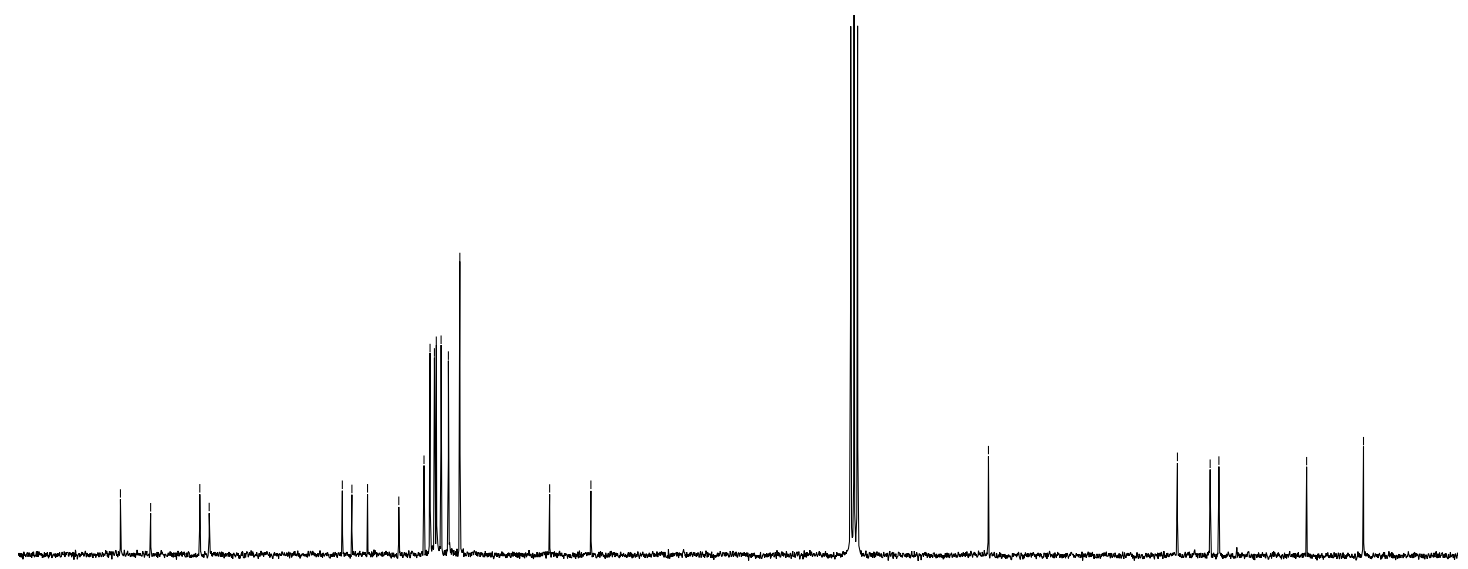

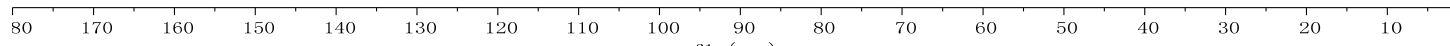


<smiles>CCOC(=O)/C(Cc1ccccc1)=C1/CC(c2ccccc2OC)c2sc(-c3ccccc3)nc2O1</smiles>
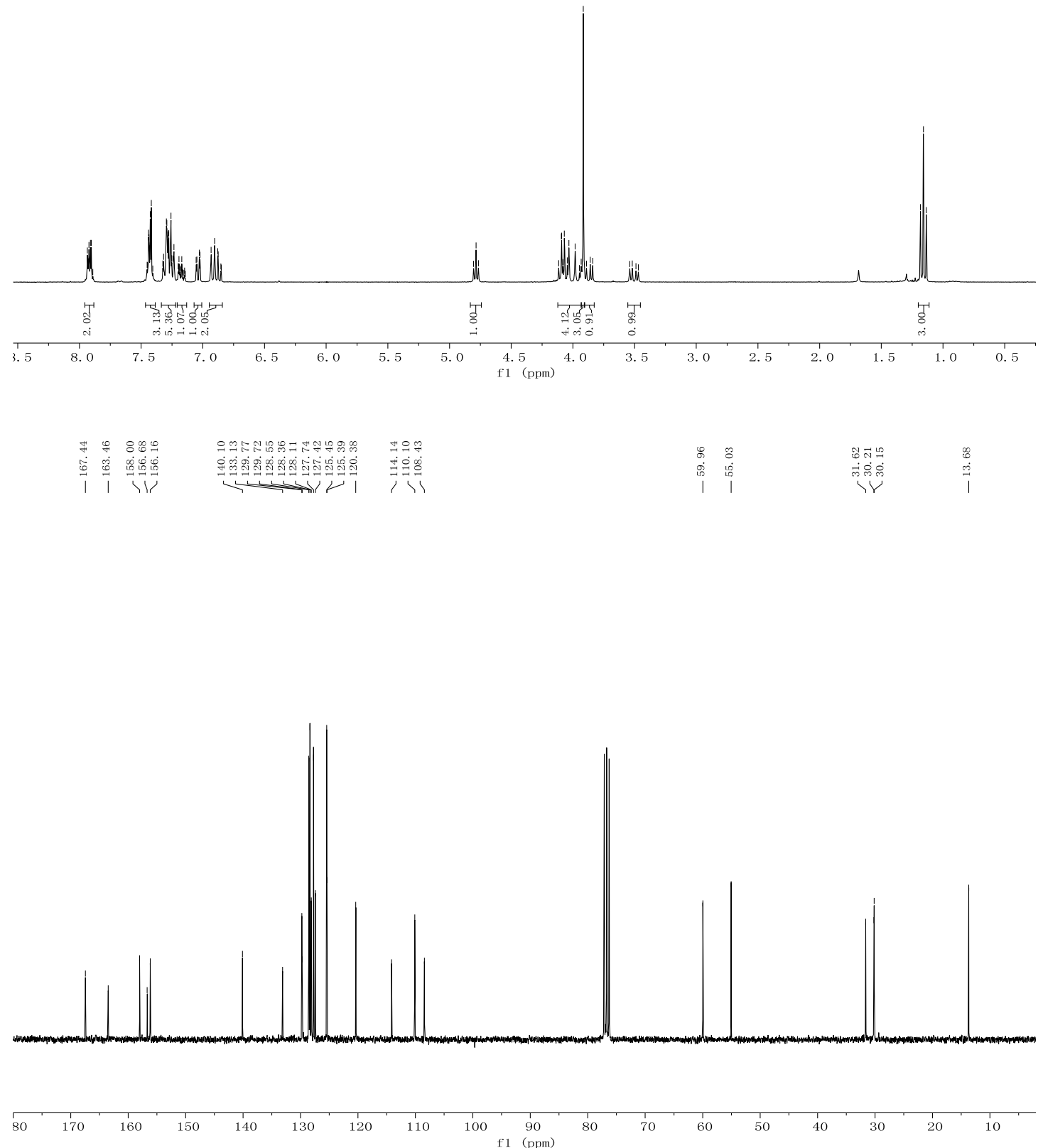
<smiles>CCOC(=O)/C(Cc1ccccc1)=C1/CC(c2ccc(OC)cc2)c2sc(-c3ccccc3)nc2O1</smiles>

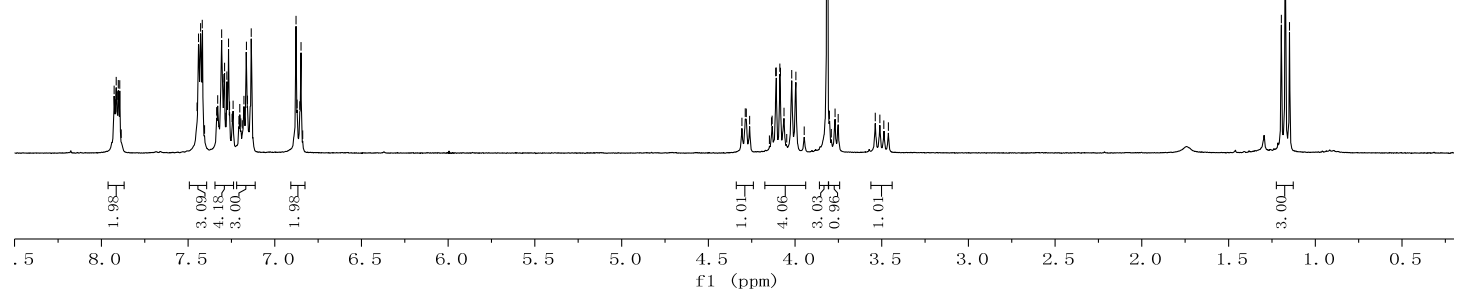

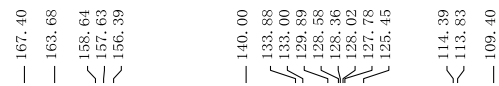

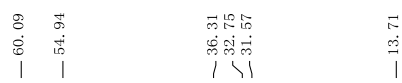
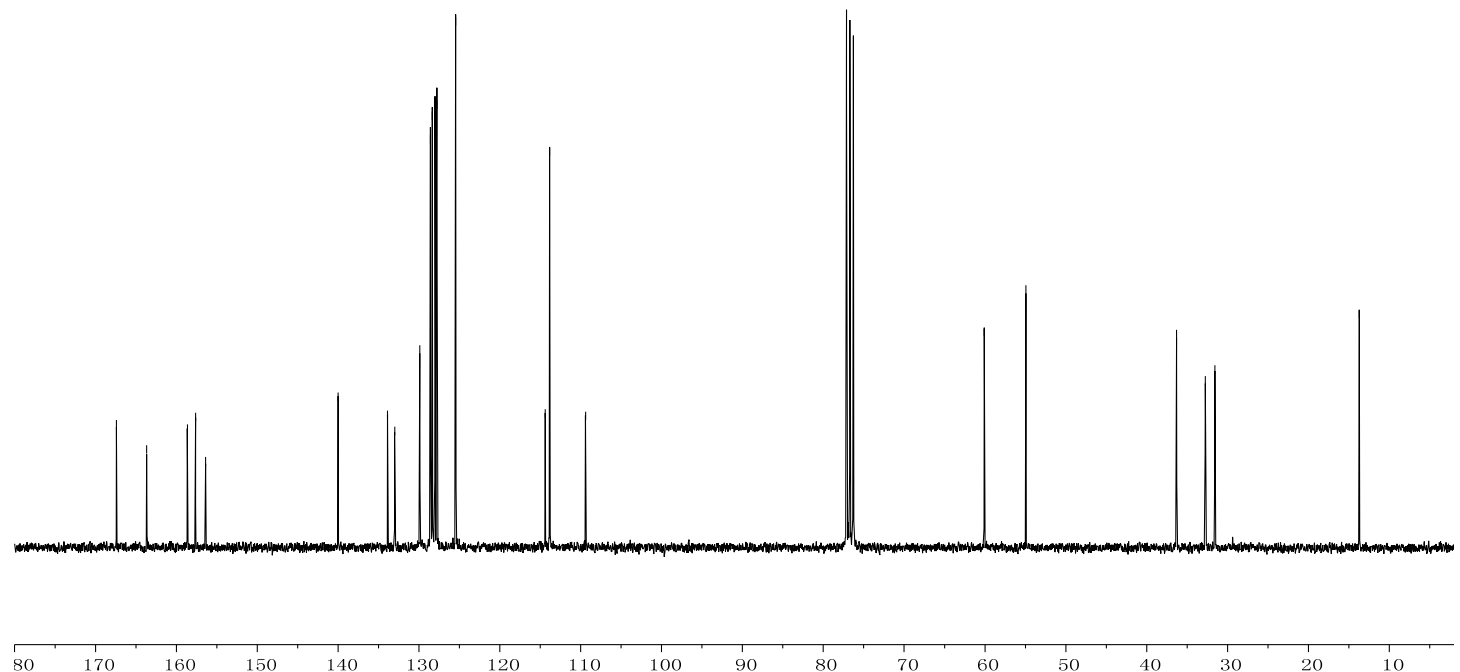

120

100

90

$80 \quad 70 \quad 60 \quad 50$

$+30$

$20 \quad 10$ 


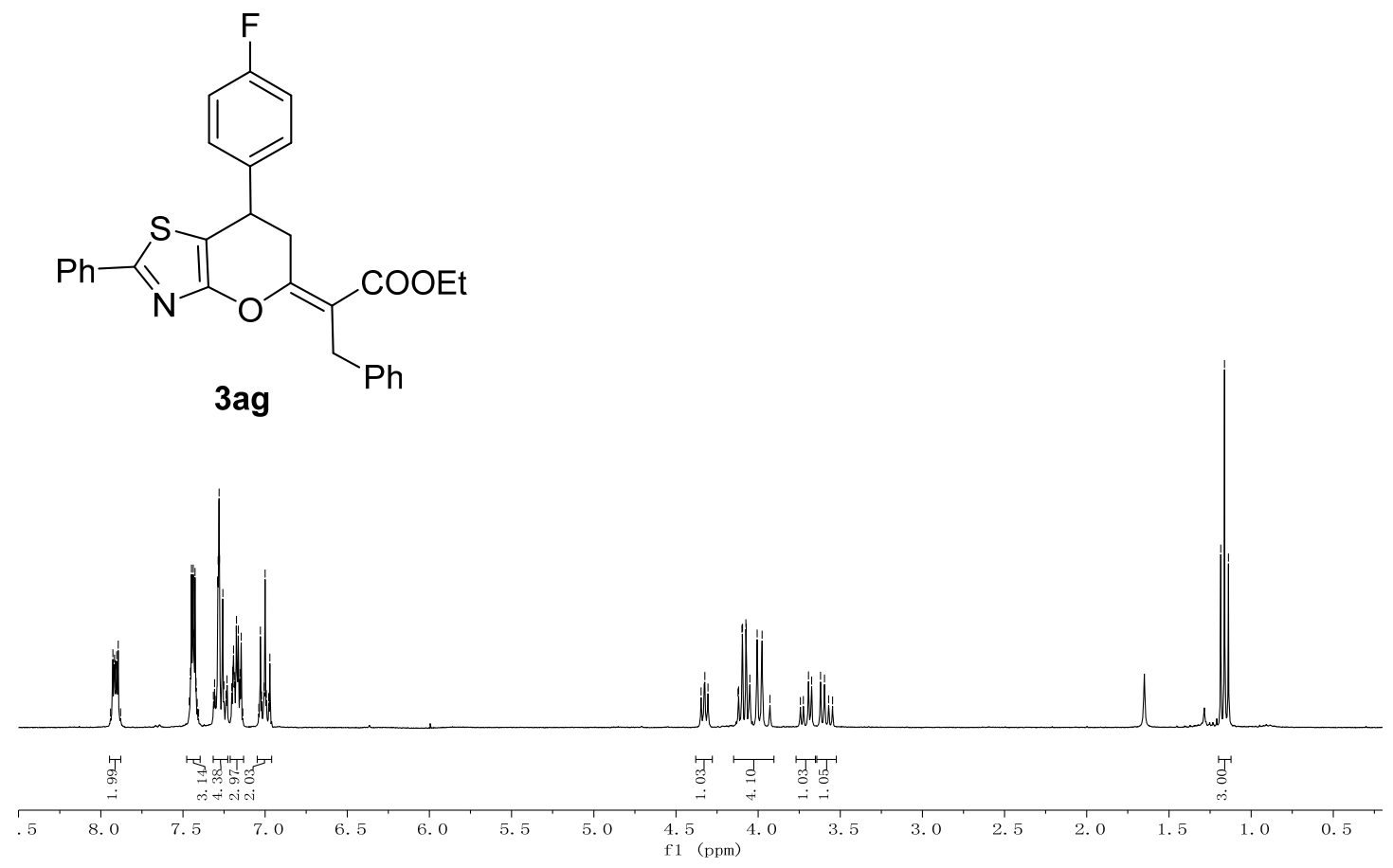

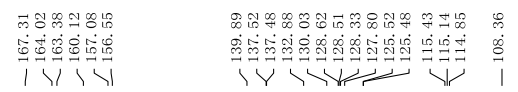

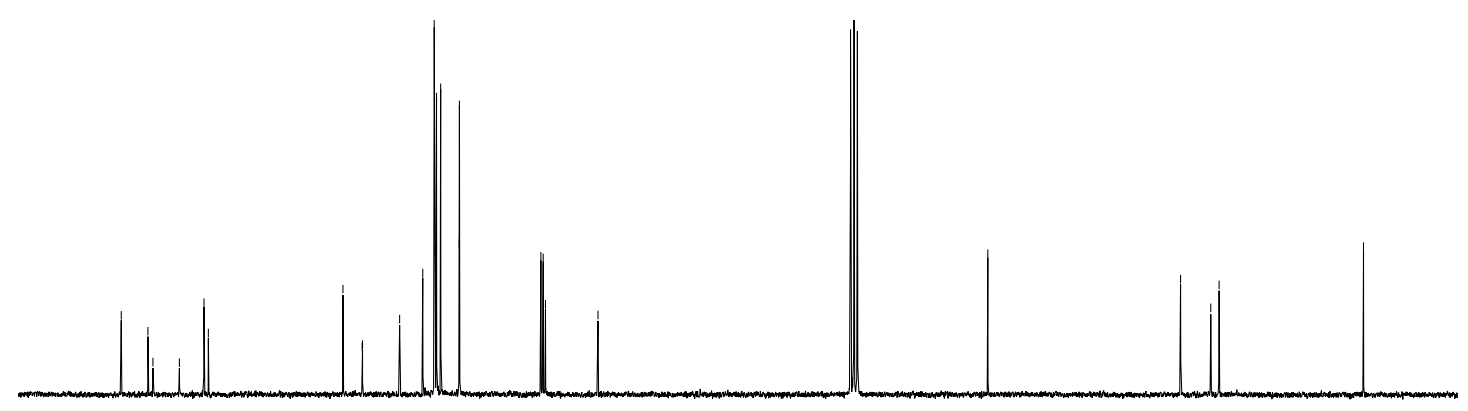

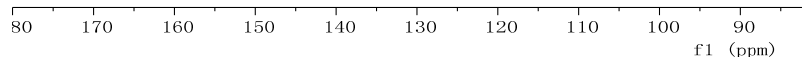


<smiles>CCOC(=O)/C(Cc1ccccc1)=C1/CC(c2ccccc2Cl)c2sc(-c3ccccc3)nc2O1</smiles>

3ah
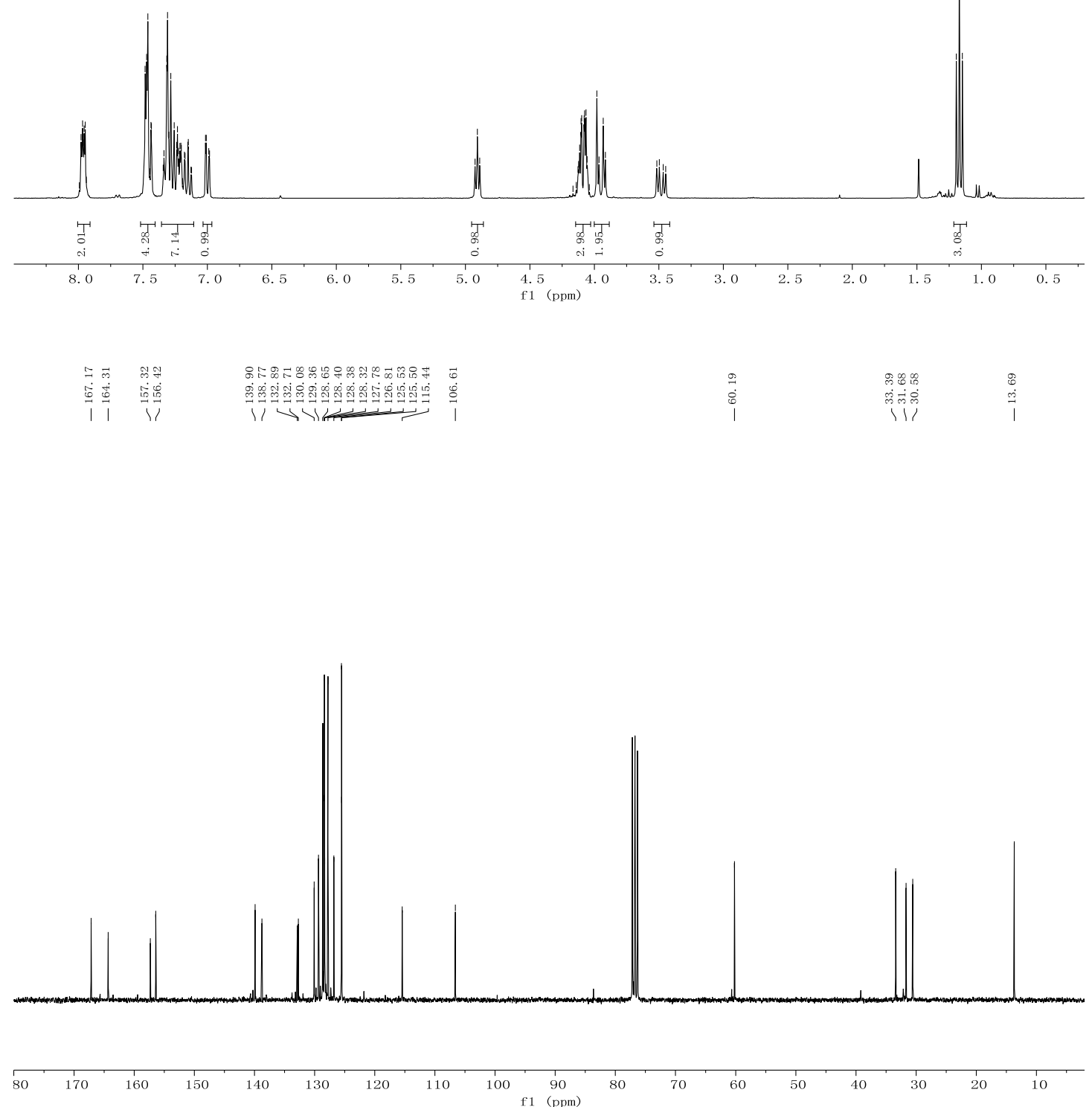
<smiles>CCOC(=O)/C(Cc1ccccc1)=C1/CC(c2ccc(Cl)cc2)c2sc(-c3ccccc3)nc2O1</smiles>

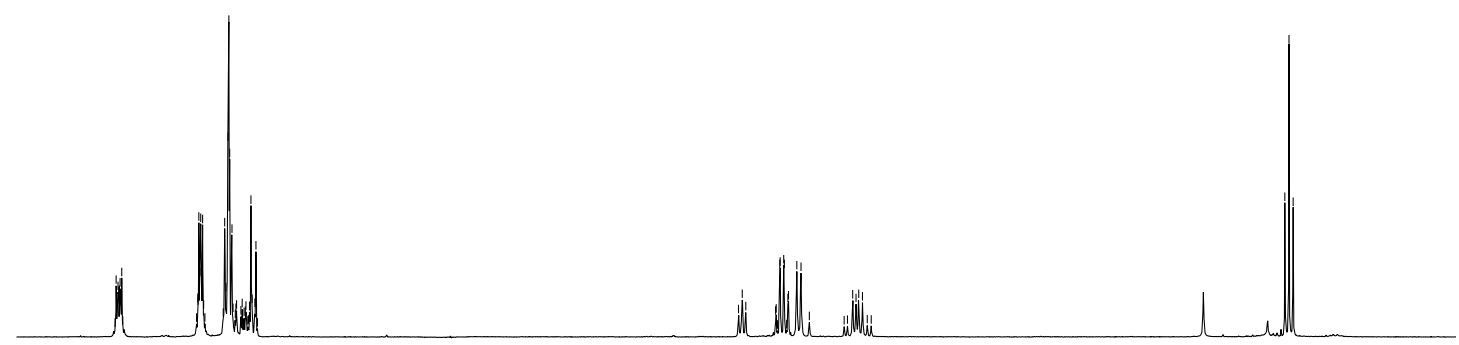

\begin{tabular}{|c|c|c|c|c|c|c|c|c|c|c|c|c|c|c|c|}
\hline & $\begin{array}{l}\text { Ti } \\
\text { ì } \\
\text { i }\end{array}$ & 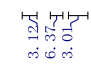 & & & & & & & $\begin{array}{l}\text { d } \\
+ \\
+\end{array}$ & $\begin{array}{l}\text { गु } \\
\text { के } \\
\text { ते }\end{array}$ & & & & & \\
\hline & 8. 0 & 7.5 & 7. 0 & 6.5 & 6. 0 & 5.5 & 5. 0 & $\begin{array}{l}4.5 \\
\text { f1 (ppm) }\end{array}$ & 1) & 3.5 & 3. 0 & 2.5 & 2. 0 & 1.5 & 1. 0 \\
\hline
\end{tabular}

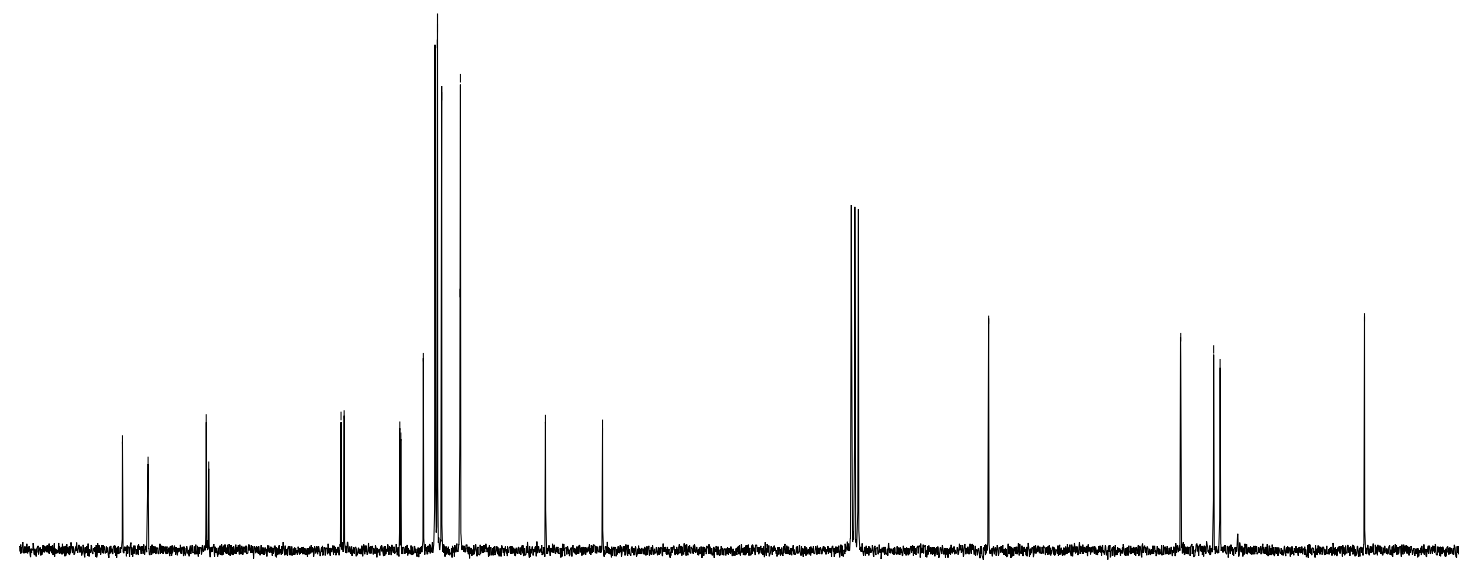

80

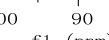

$80 \quad 70 \quad 60 \quad 50$

30

$10 \div \quad 10$

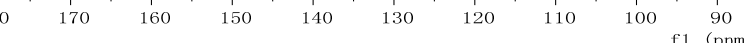


<smiles>CCOC(=O)/C(Cc1ccccc1)=C1/CC(c2ccc(Br)cc2)c2sc(-c3ccccc3)nc2O1</smiles>
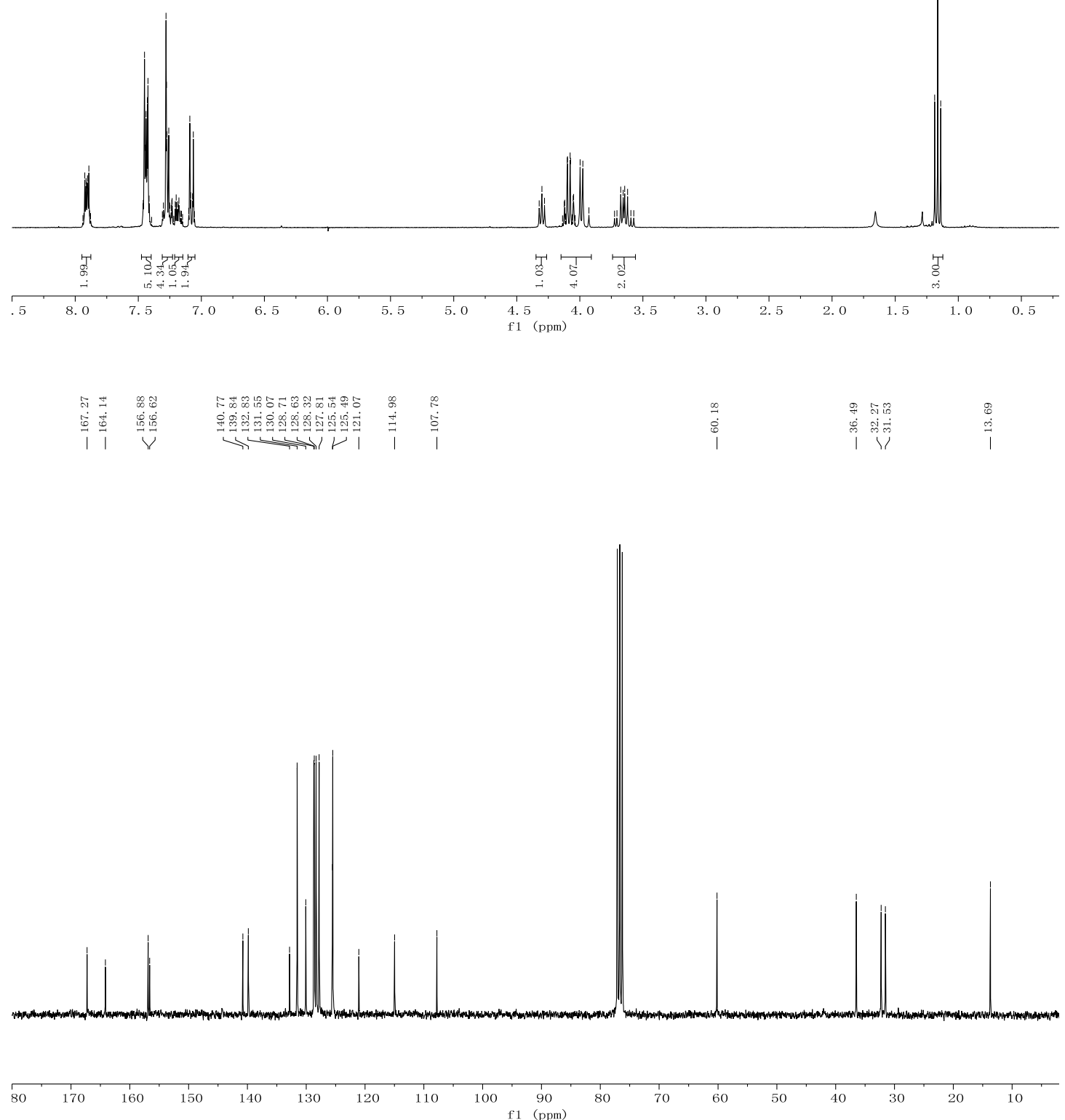
<smiles>CCOC(=O)C(Cc1ccccc1)=C1CC(c2ccc(C#N)cc2)c2sc(-c3ccccc3)nc2O1</smiles>

(1)

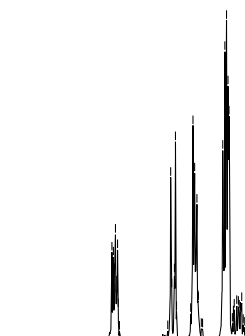

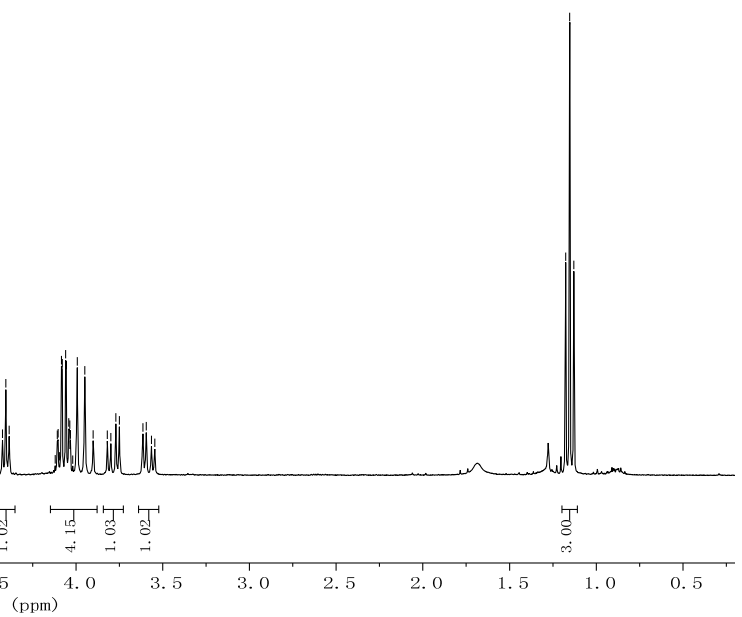

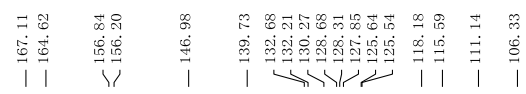

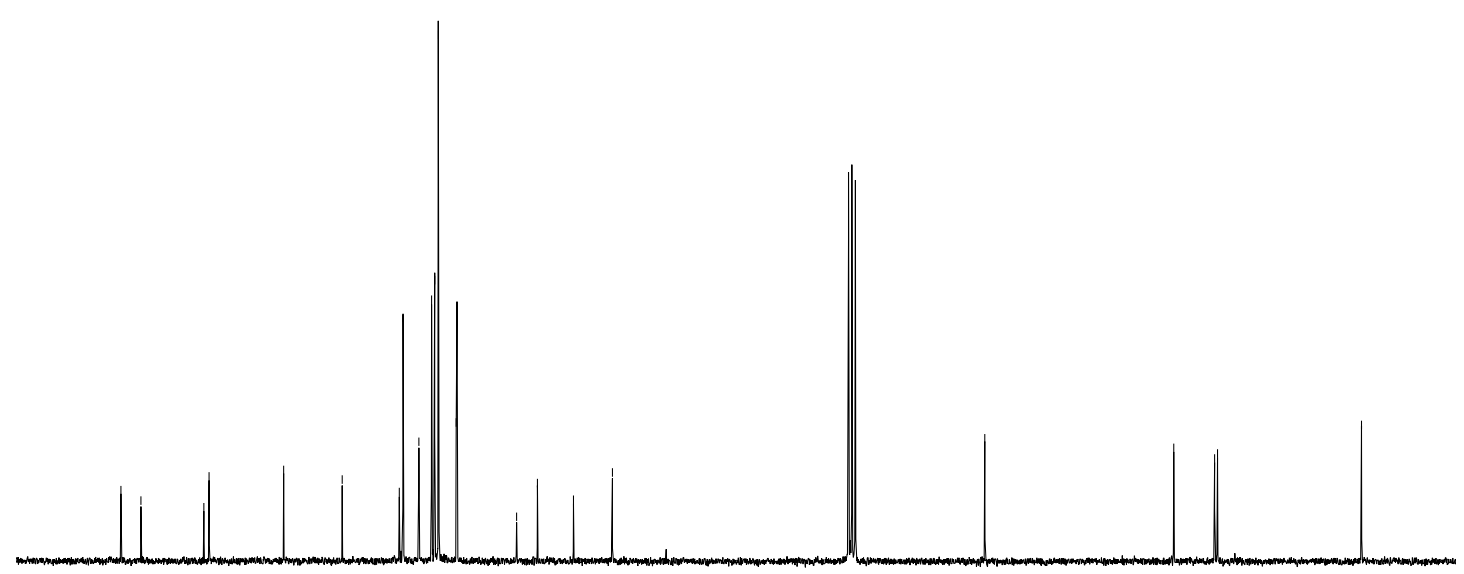

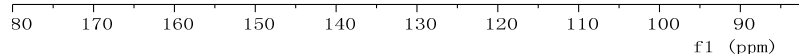


<smiles>CCOC(=O)/C(Cc1ccccc1)=C1/CC(c2ccc(Cl)c(Cl)c2)c2sc(-c3ccccc3)nc2O1</smiles>
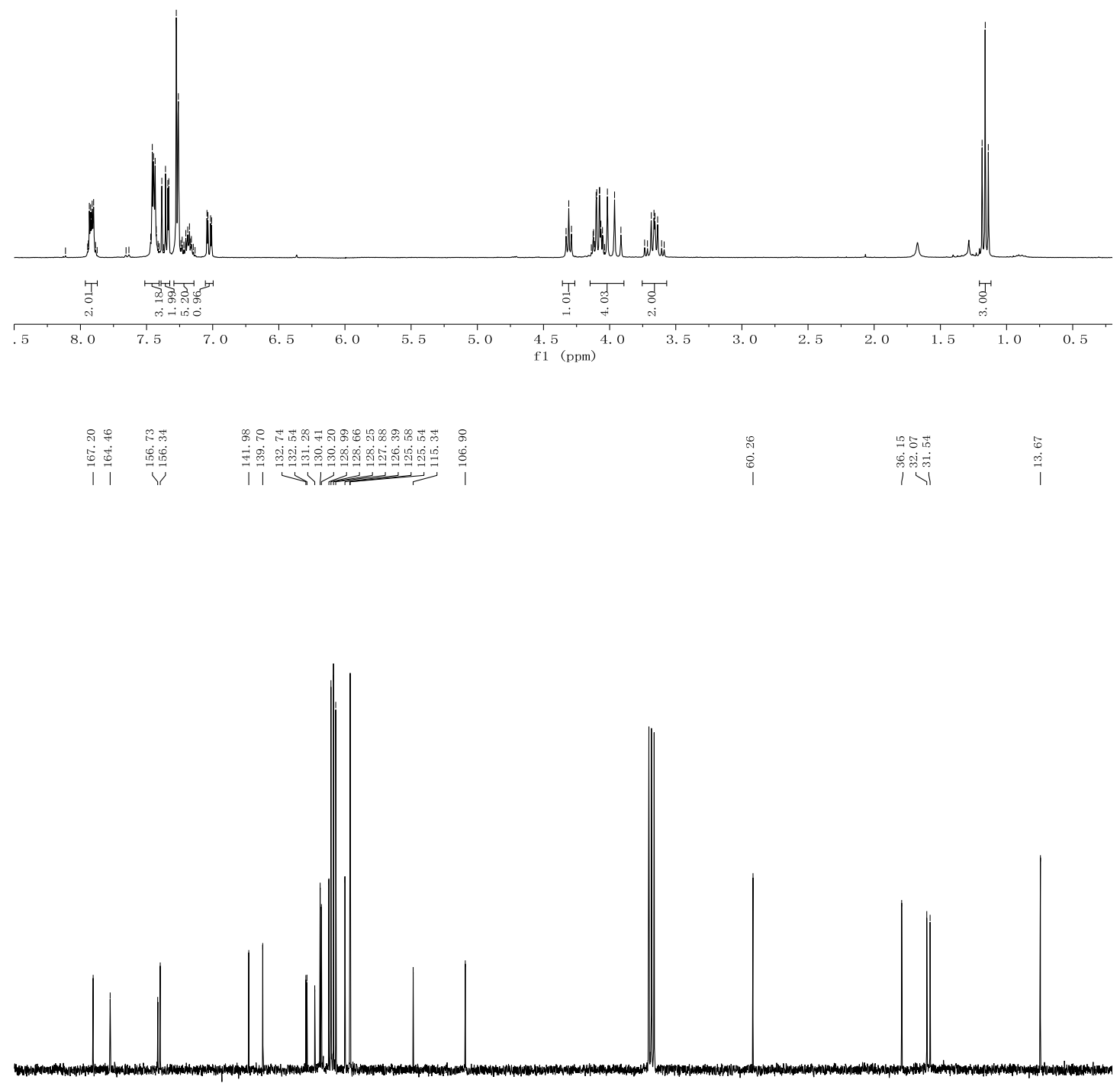

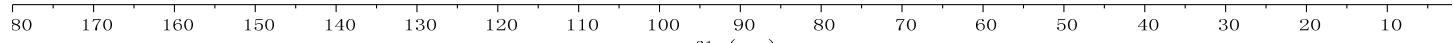


<smiles>CCOC(=O)/C(Cc1ccccc1)=C1/CC(c2ccc3ccccc3c2)c2sc(-c3ccccc3)nc2O1</smiles>
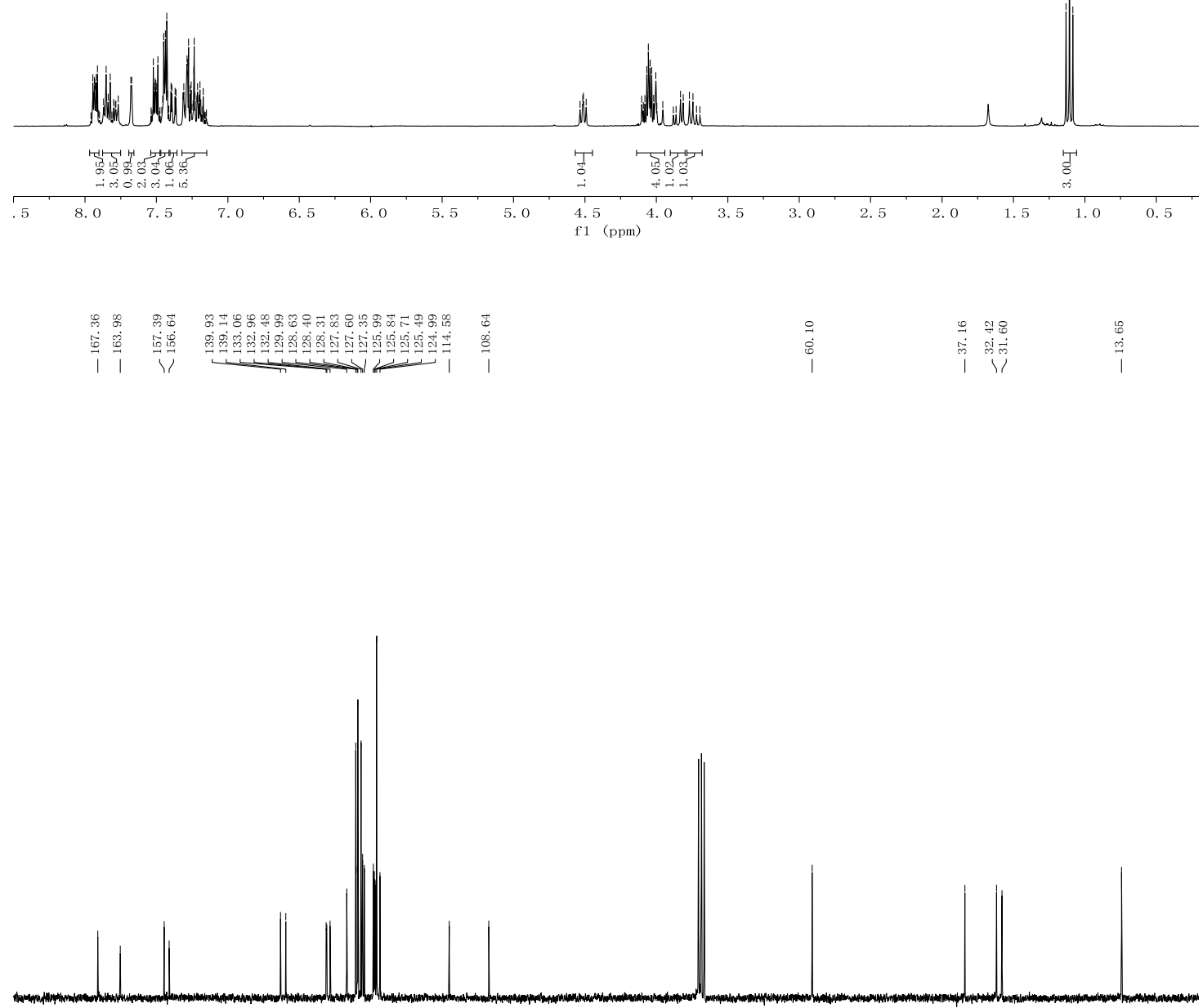

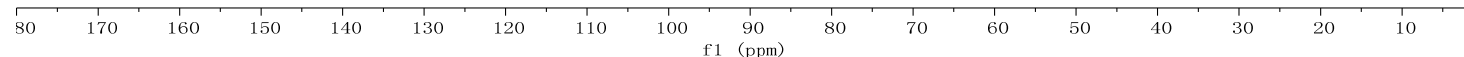


<smiles>CCOC(=O)/C(Cc1ccccc1)=C1/CC(c2cccs2)c2sc(-c3ccccc3)nc2O1</smiles>

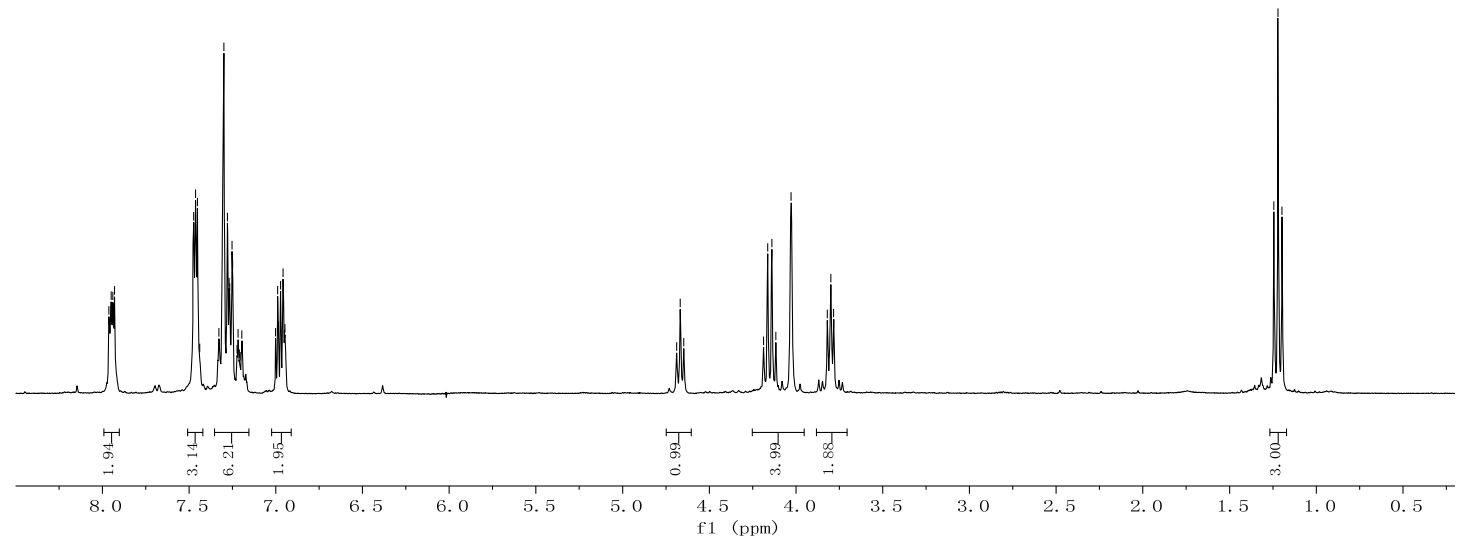

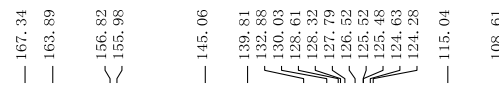

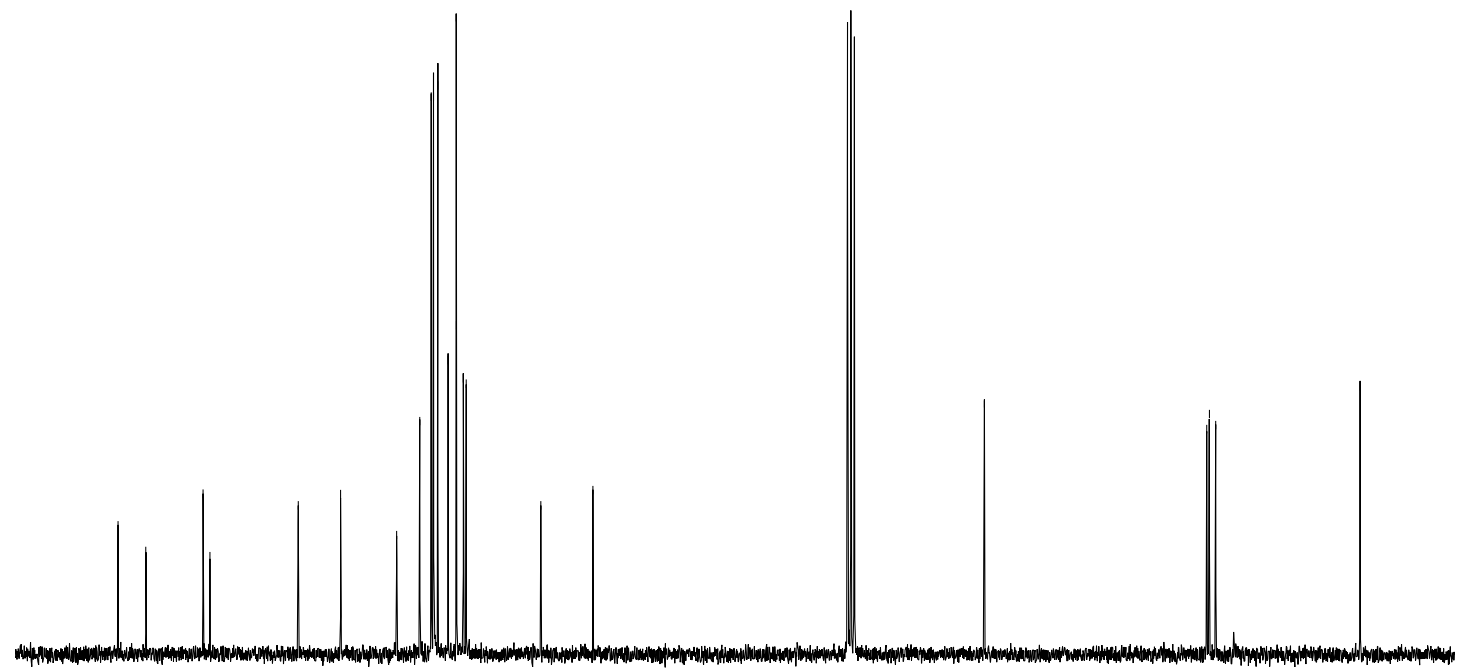

80

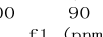

$80 \quad 70 \quad 60$

30 $10 \div \quad 10$ 
<smiles>CCOC(=O)C(Cc1cccc(C)c1)=C1CC(c2ccccc2)c2sc(-c3ccccc3)nc2O1</smiles>

3 ba

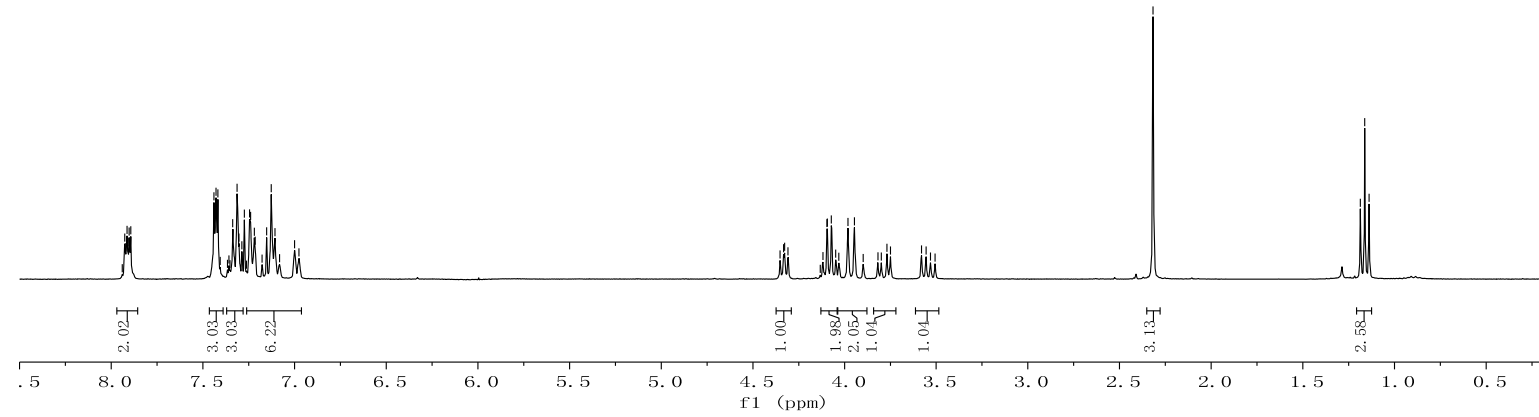

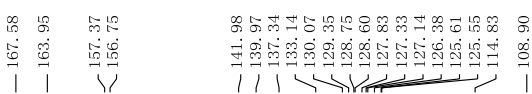

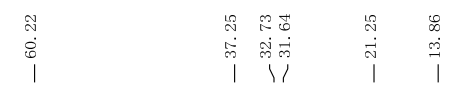

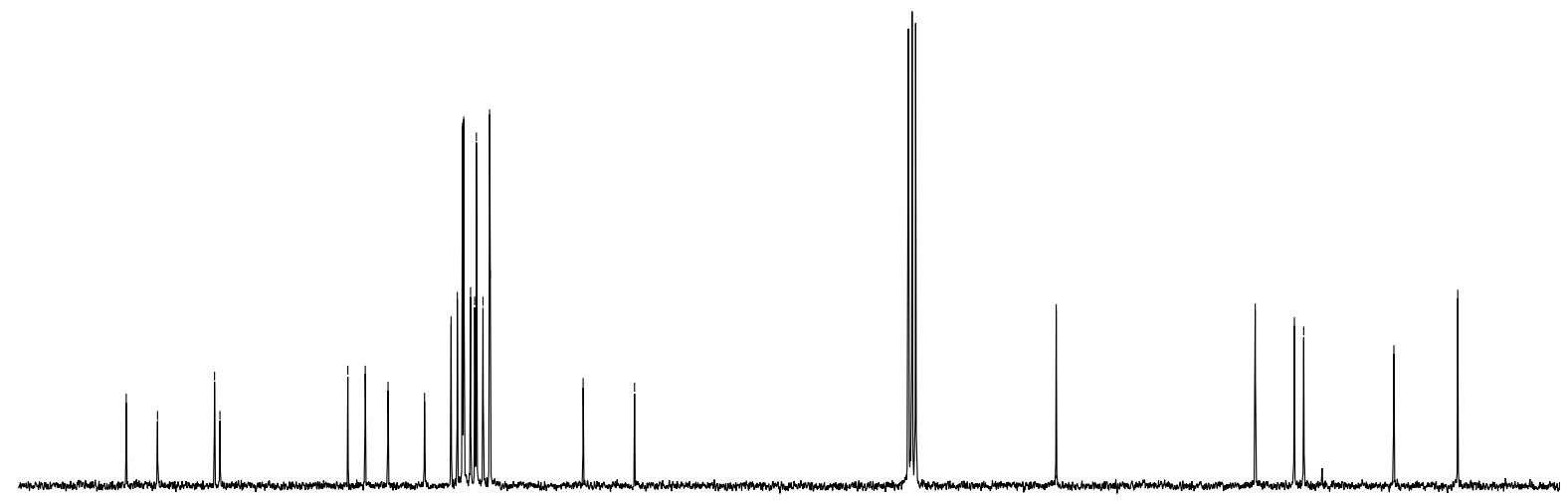

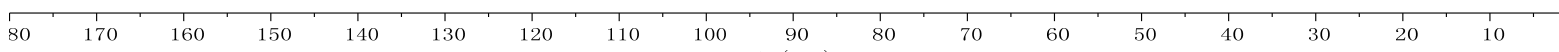
f1 (ppm) 


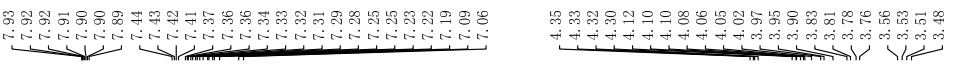

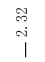

这
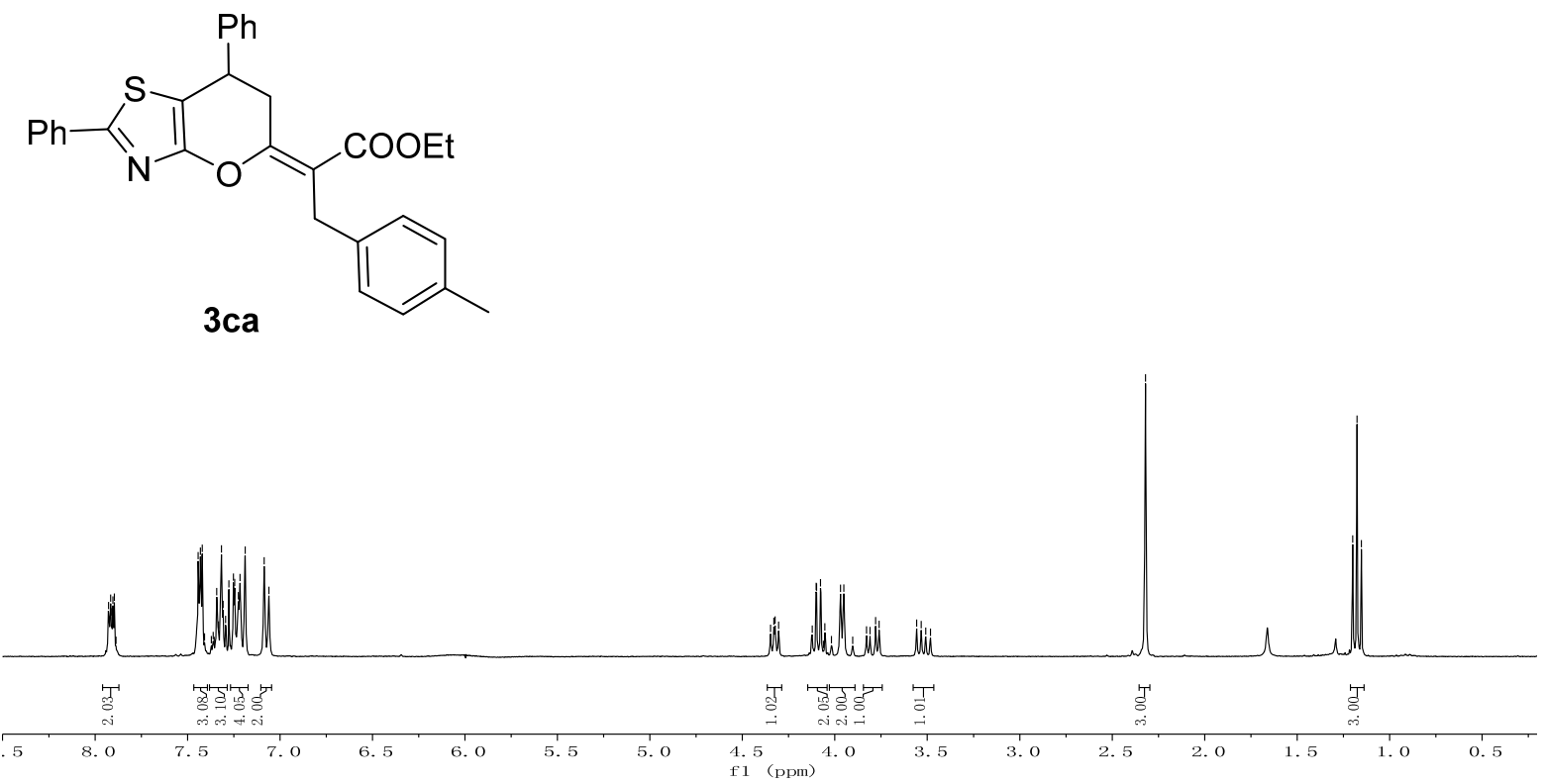

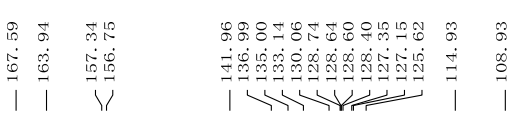
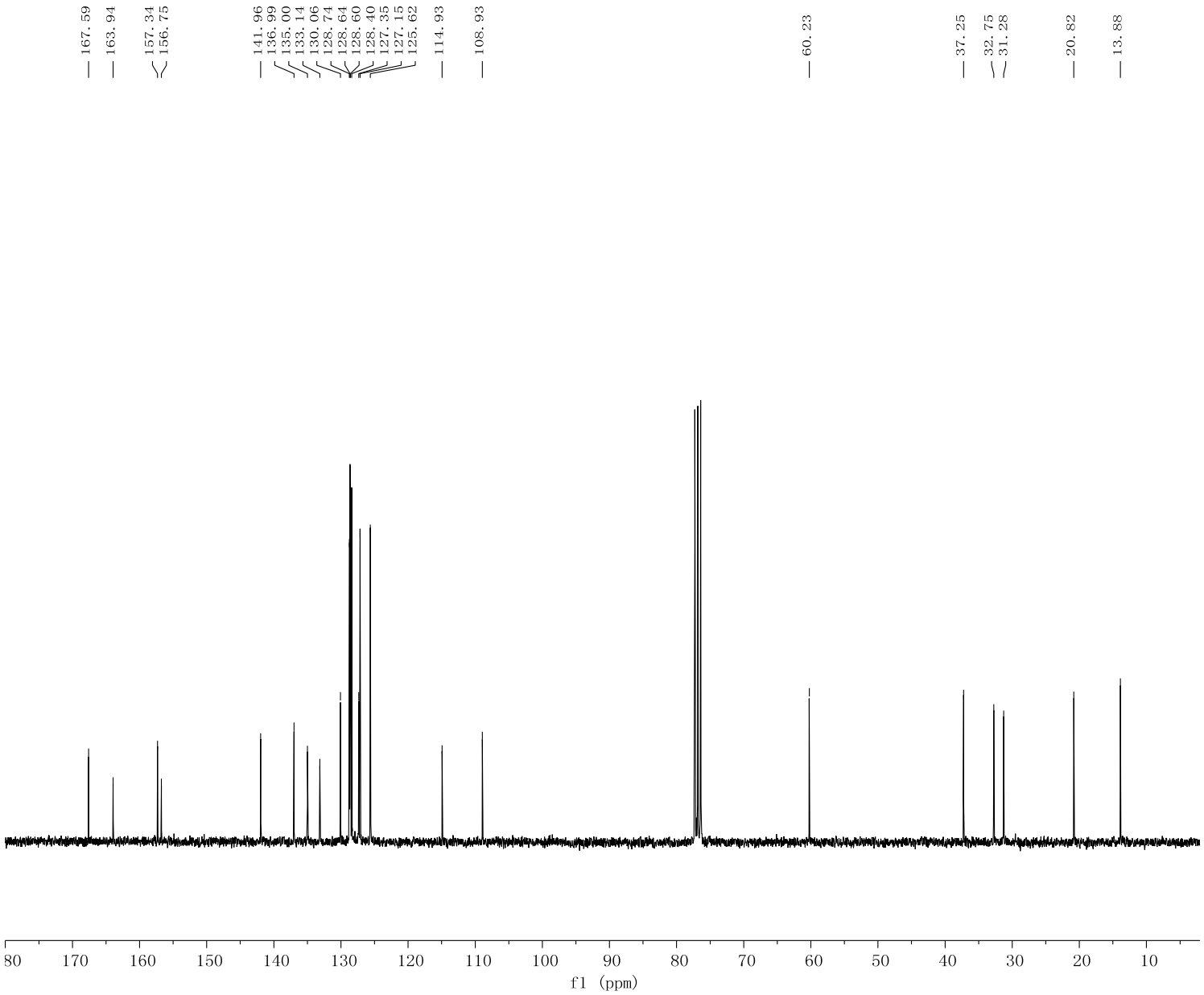

S37 
<smiles>CCOC(=O)/C(Cc1ccc(C(C)(C)C)cc1)=C1/CC(c2ccccc2)c2sc(-c3ccccc3)nc2O1</smiles>

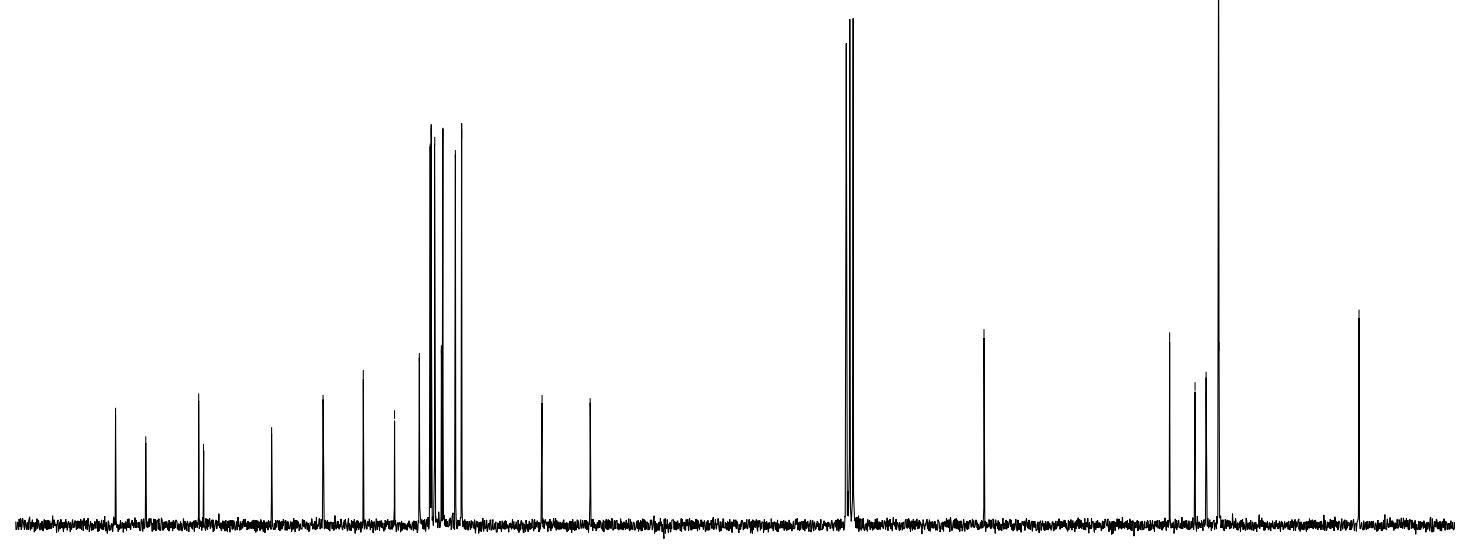

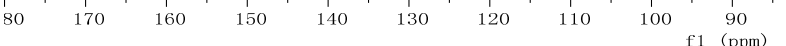


<smiles>CCOC(=O)C1=C(Cc2cc(OC)cc(OC)c2)Oc2nc(-c3ccccc3)sc2C1c1ccccc1</smiles>

3ea$$
\text { - }
$$

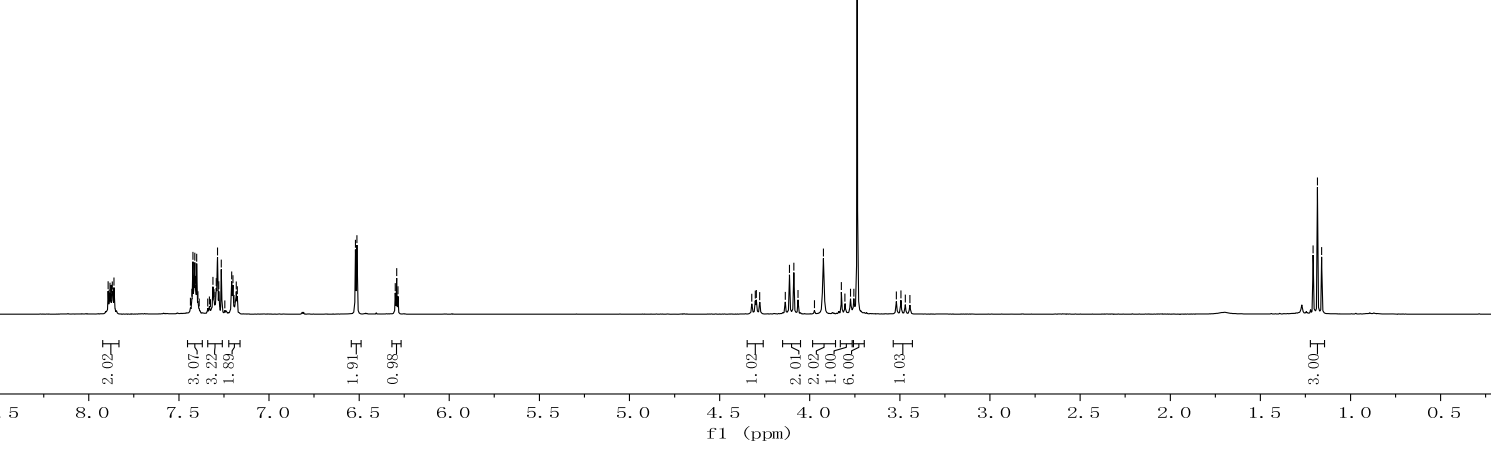

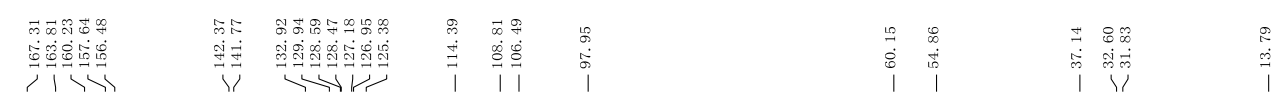

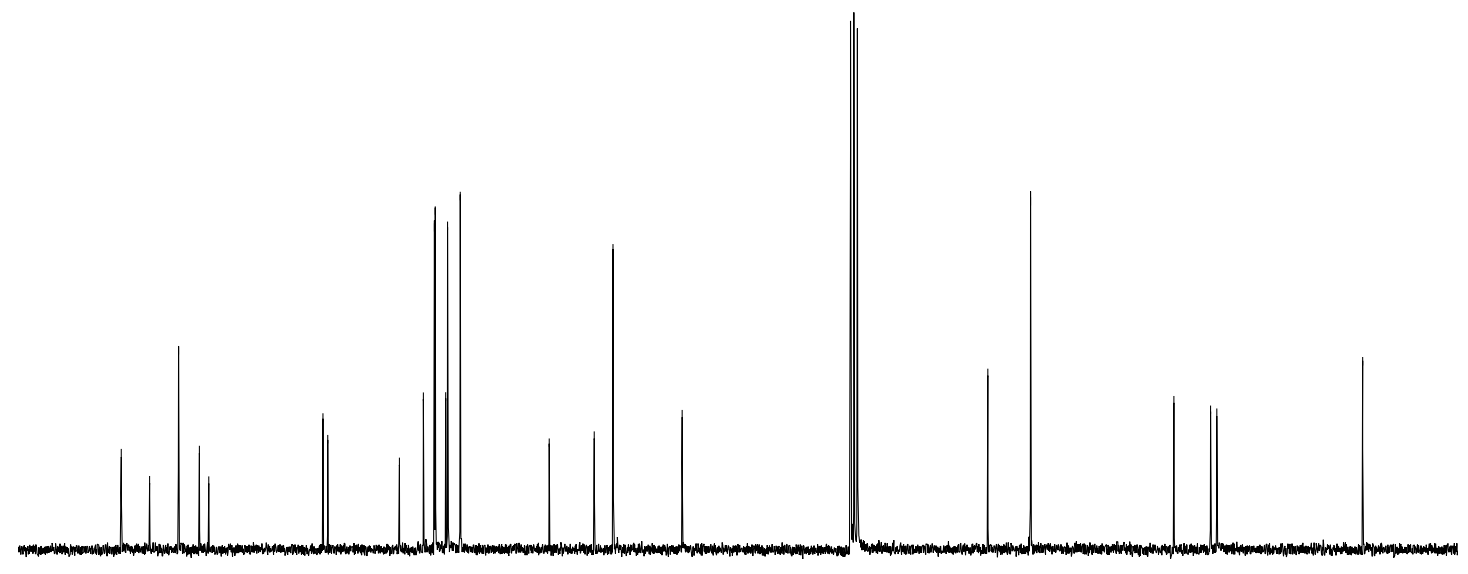

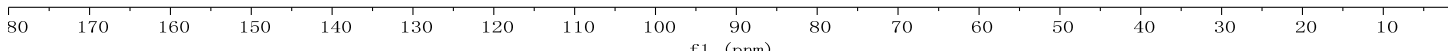




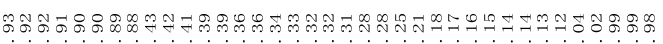

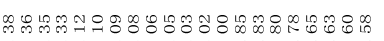

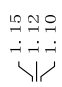
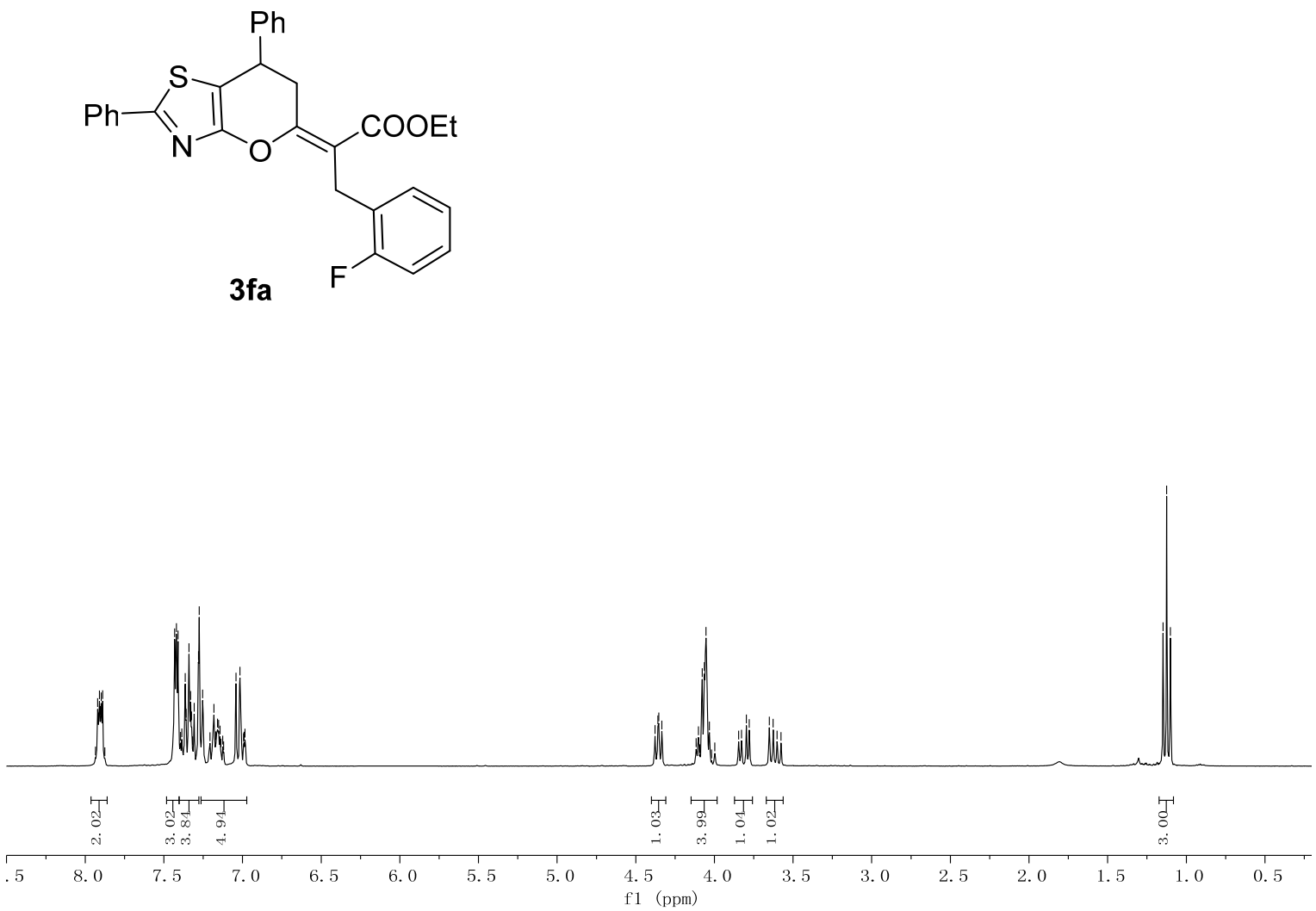

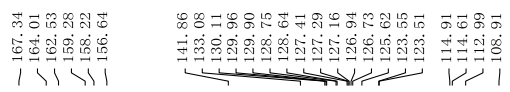
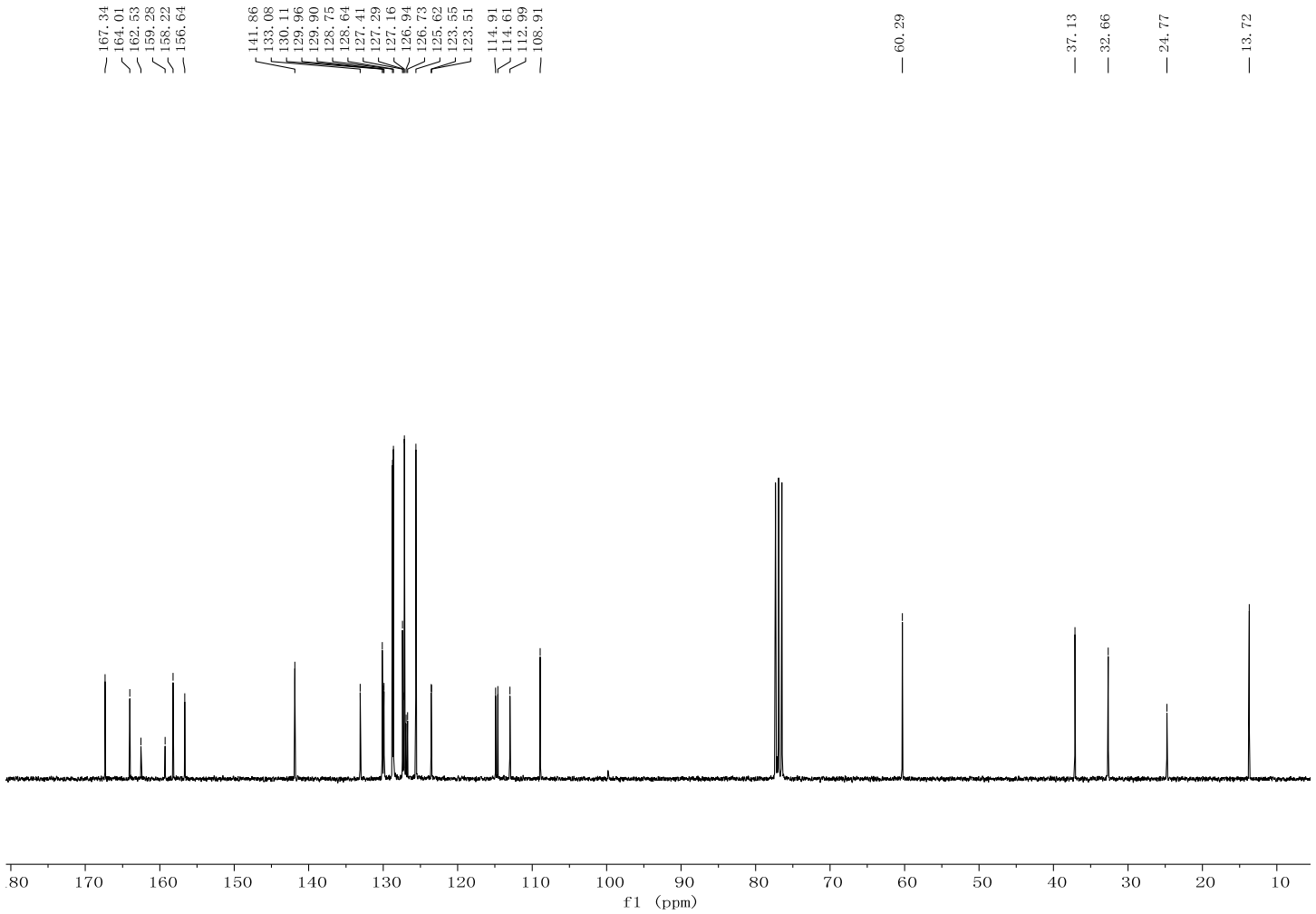

S40 


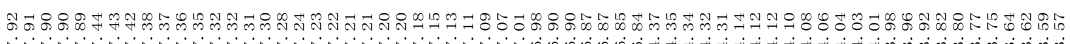

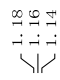
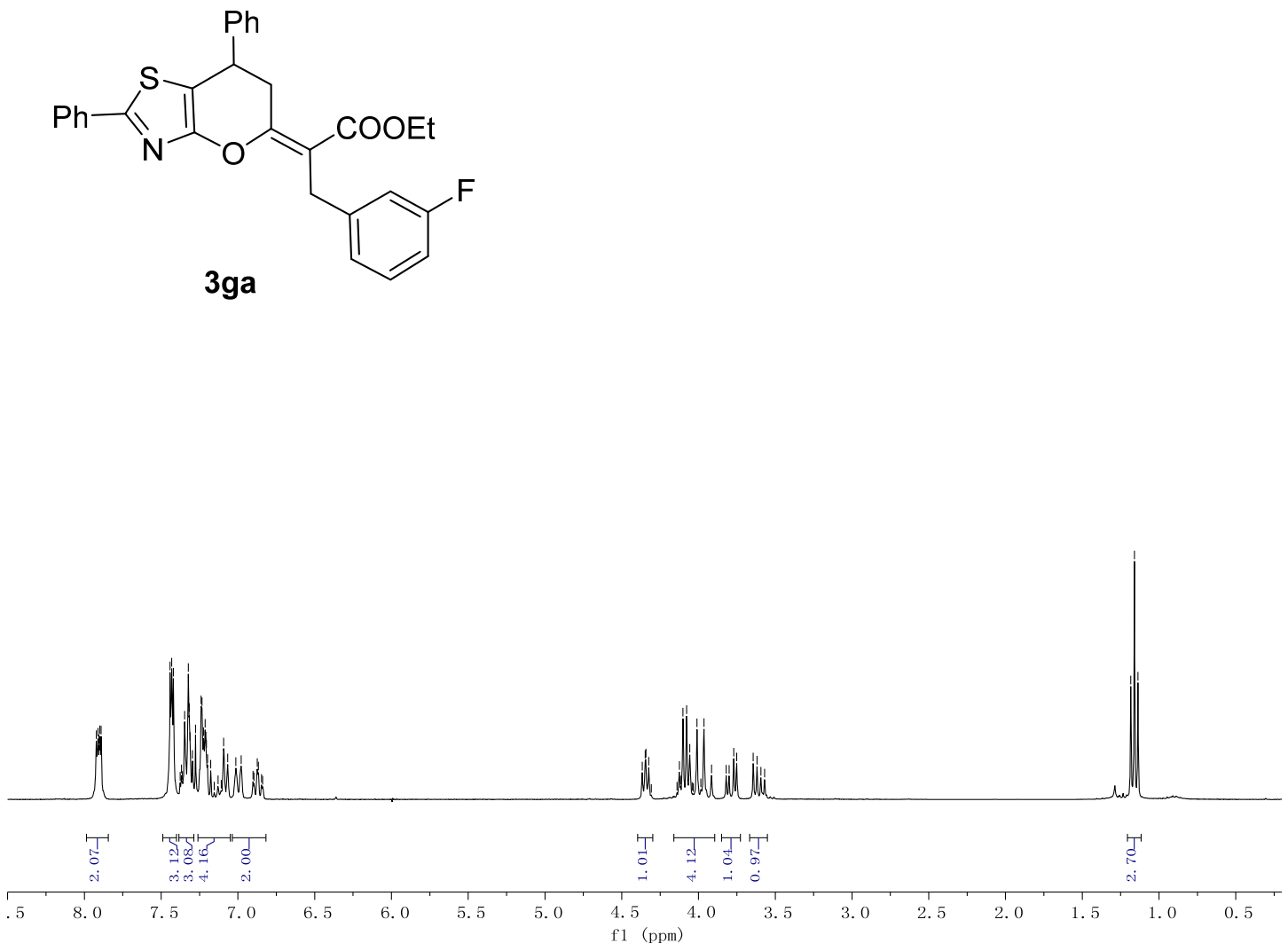

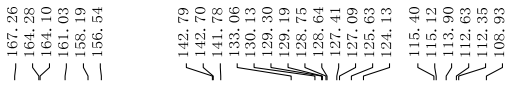

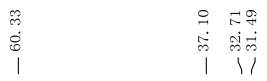

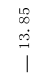
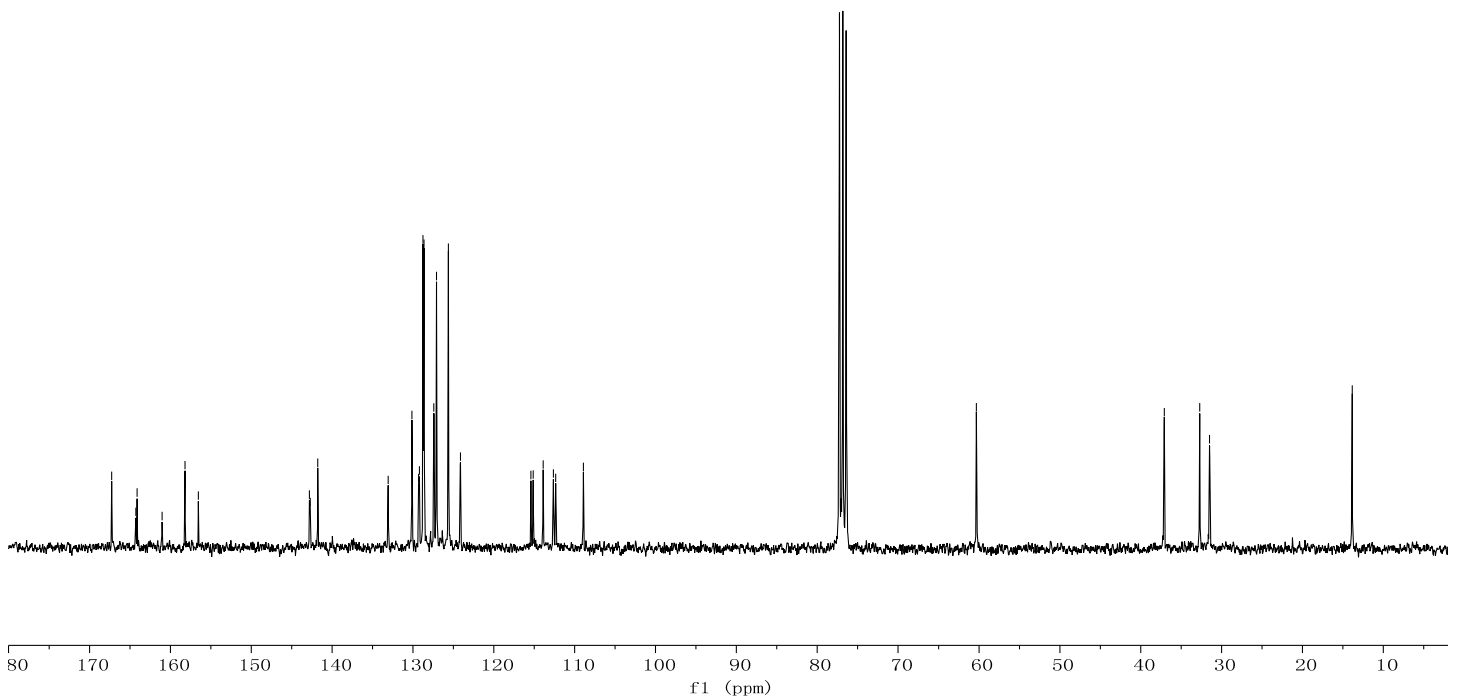

S41 


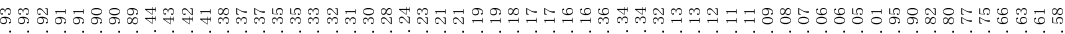

ili
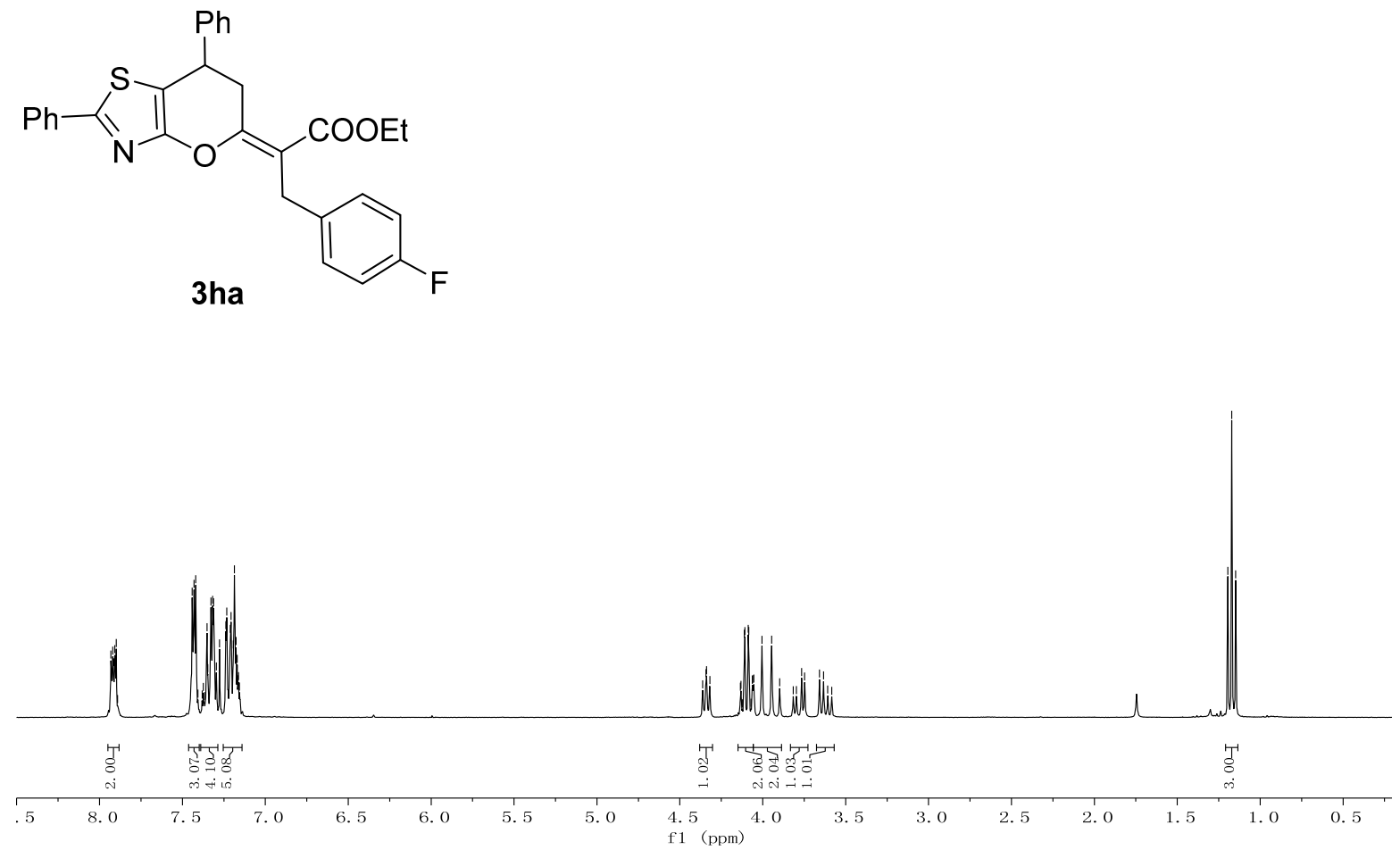

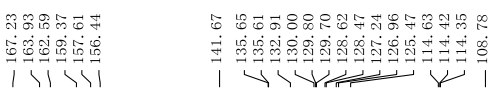

$\begin{array}{lll}0 & 0 & 0 \\ 0 & 0 & 0 \\ 0 & 0 & 0 \\ 0 & 0 & 0 \\ 0 & 1 & 1\end{array}$

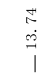
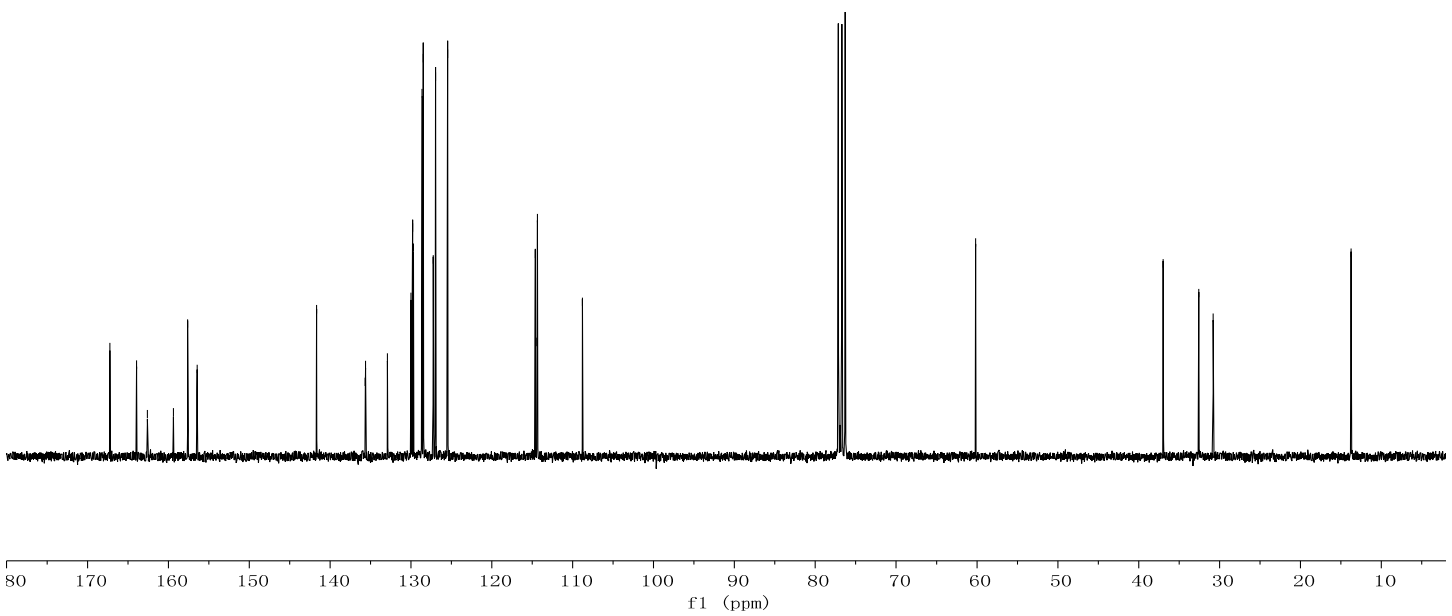

S42 


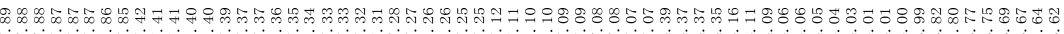

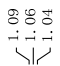
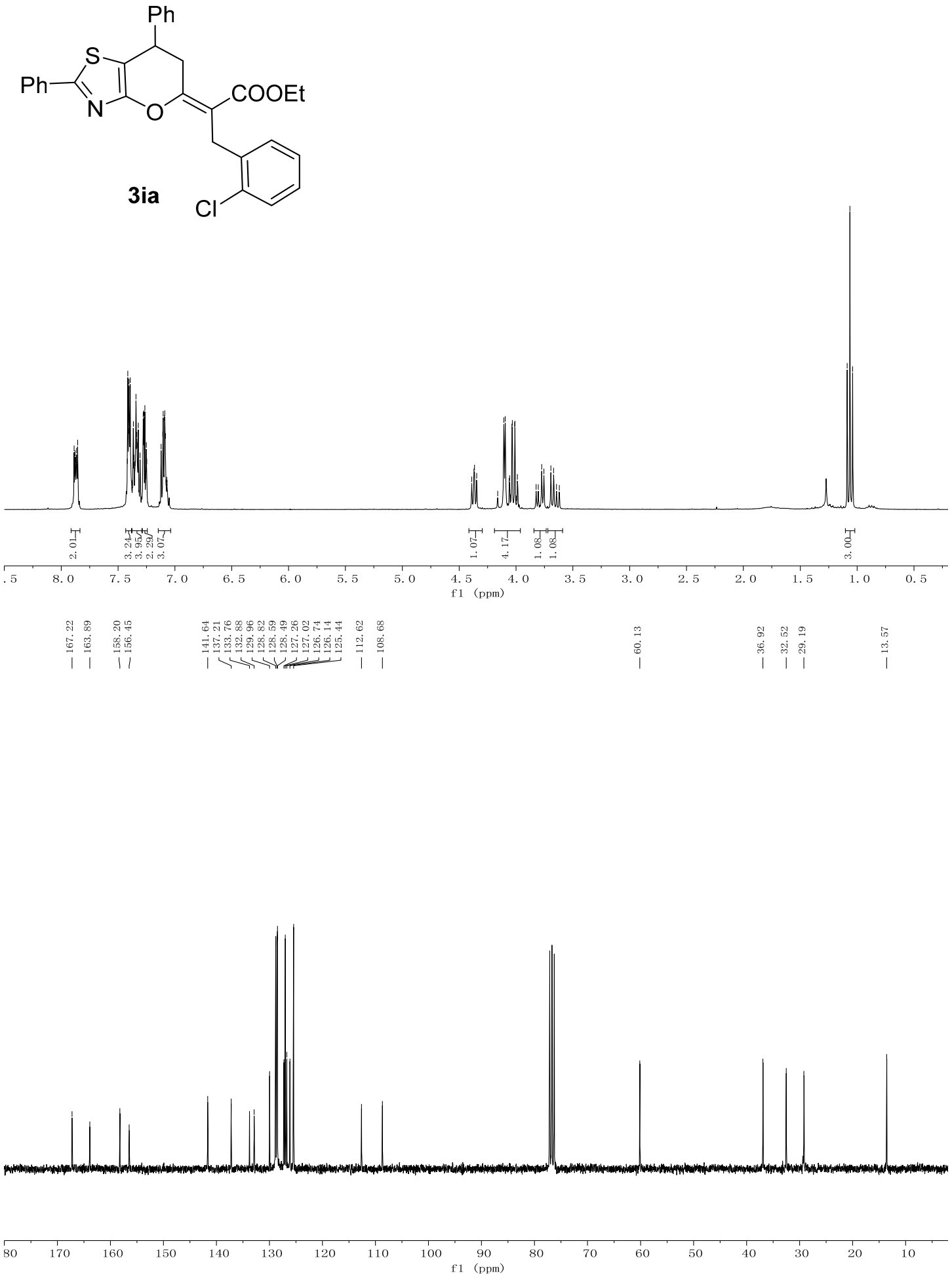

S43 
<smiles>CCOC(=O)C(Cc1cccc(Cl)c1)=C1CC(c2ccccc2)c2sc(-c3ccccc3)nc2O1</smiles>
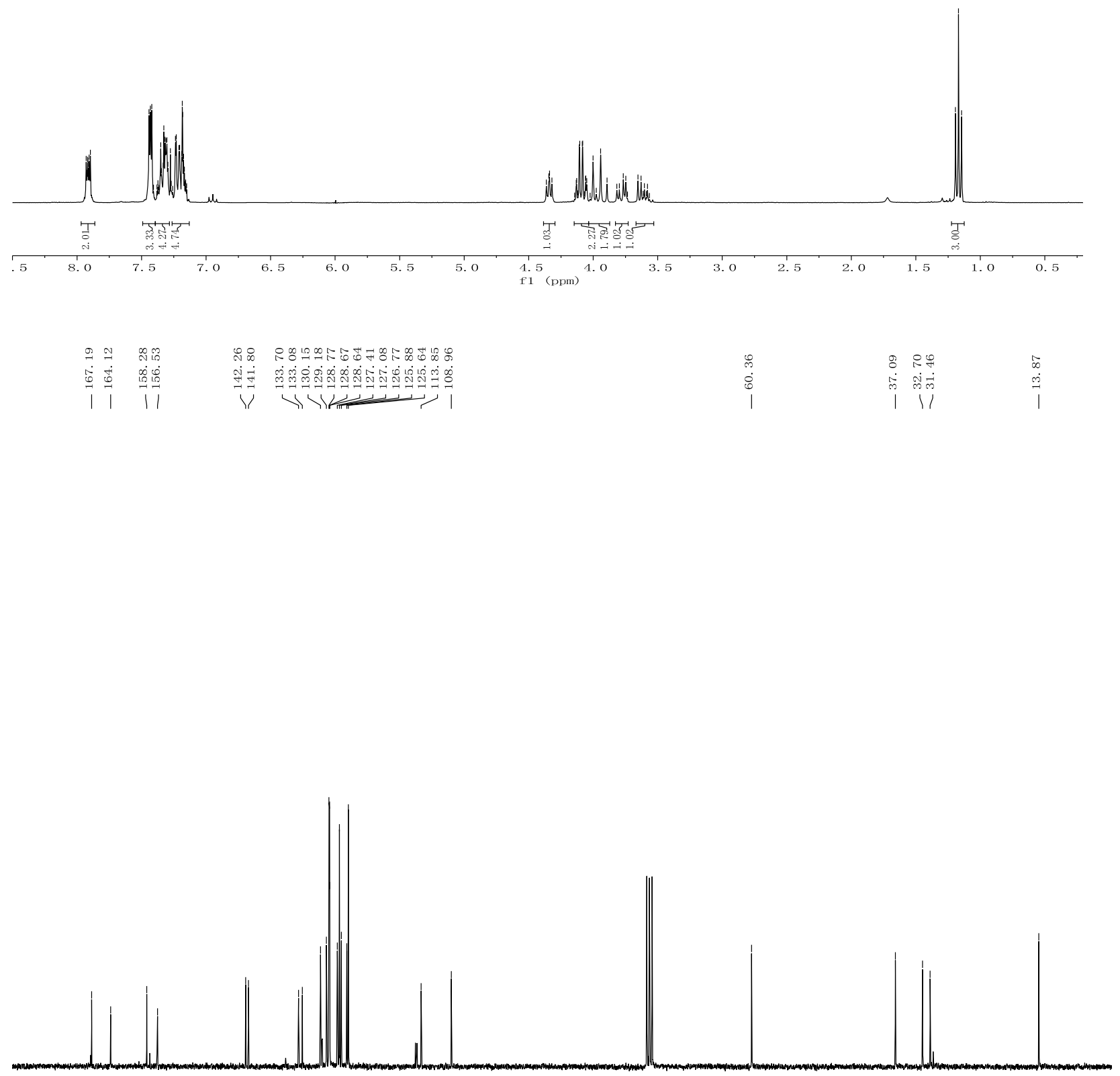

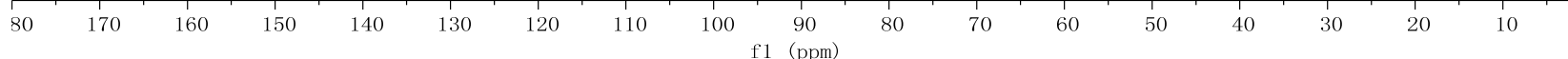



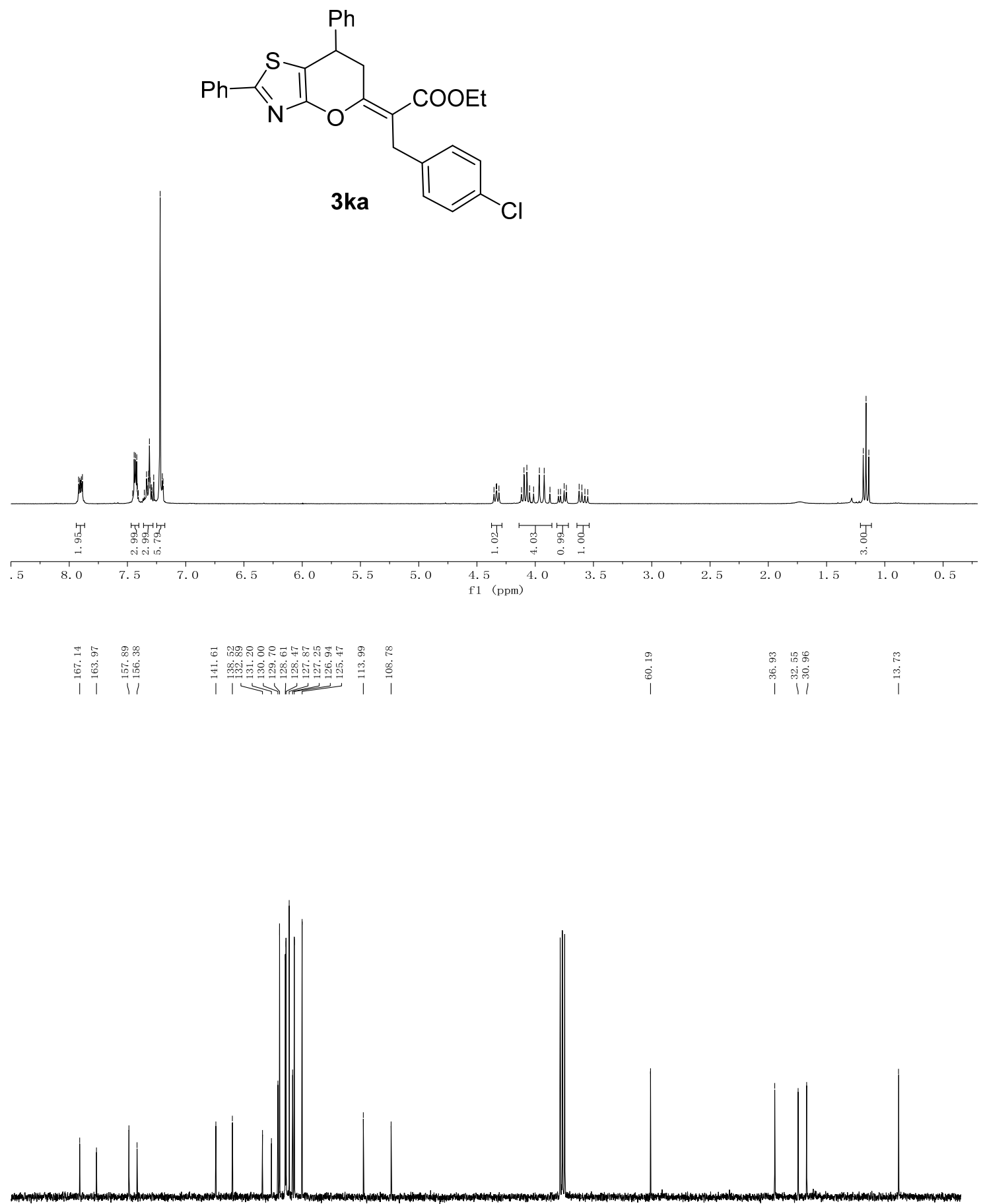

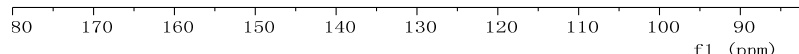


<smiles>CCOC(=O)/C(Cc1ccccc1Br)=C1/CC(c2ccccc2)c2sc(-c3ccccc3)nc2O1</smiles>

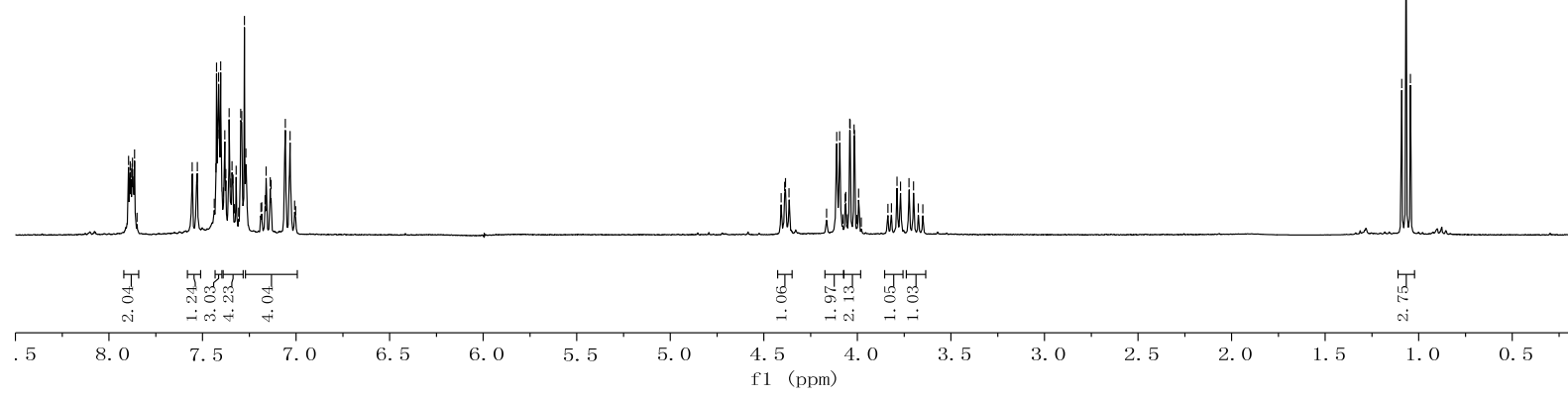

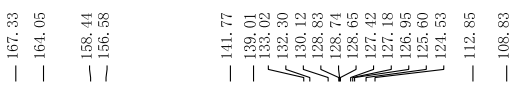
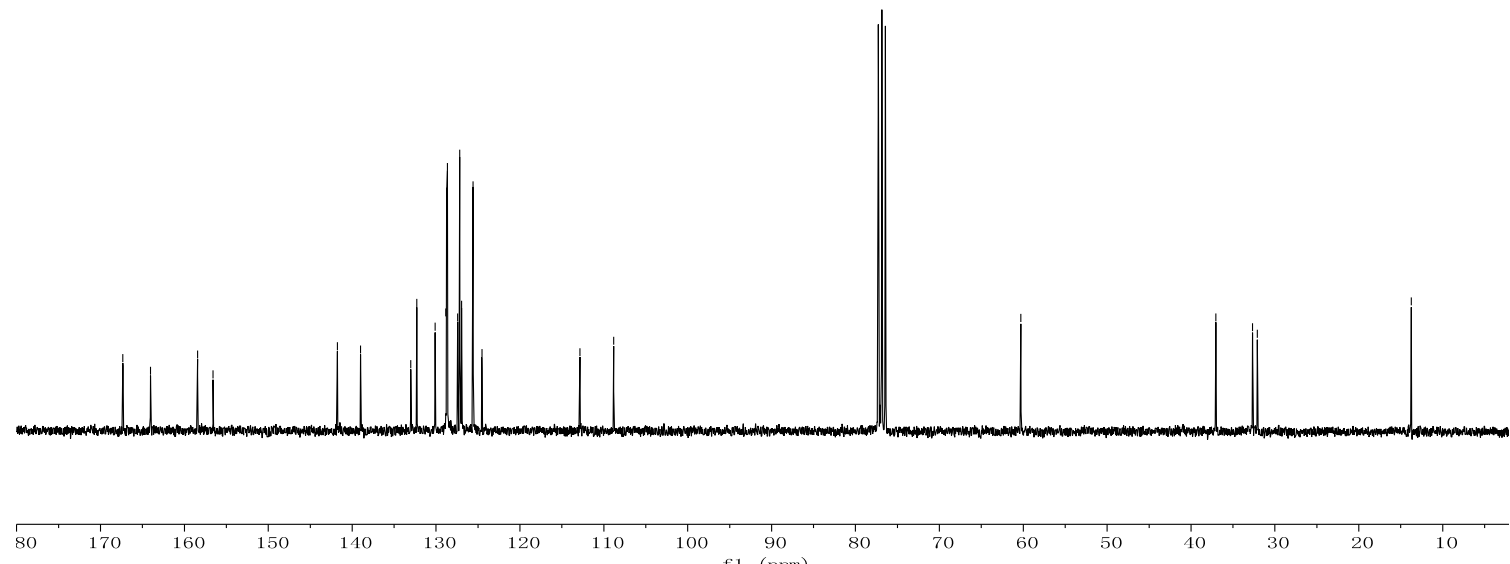
<smiles>CCOC(=O)/C(Cc1cccc(Br)c1)=C1/CC(c2ccccc2)c2sc(-c3ccccc3)nc2O1</smiles>
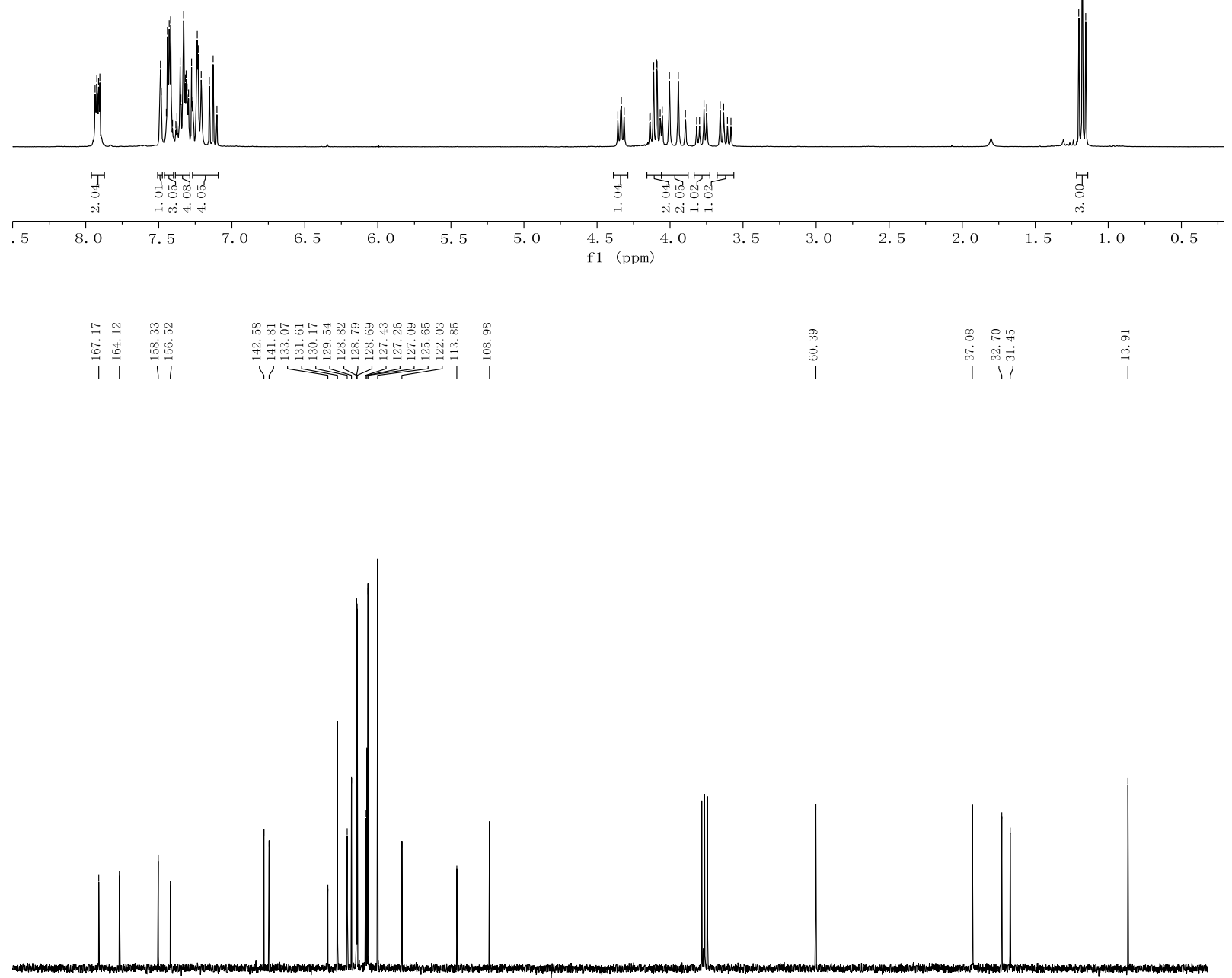
<smiles>CCOC(=O)C(Cc1ccc(Br)cc1)=C1CC(c2ccccc2)c2sc(-c3ccccc3)nc2O1</smiles>
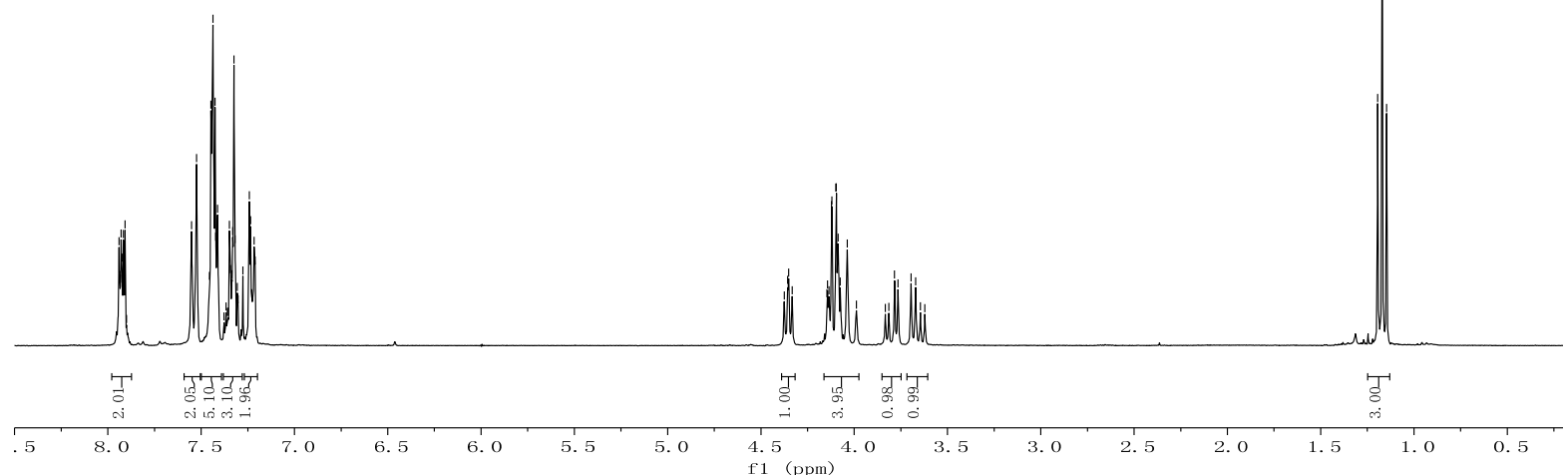

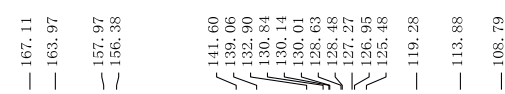

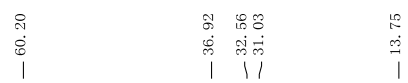

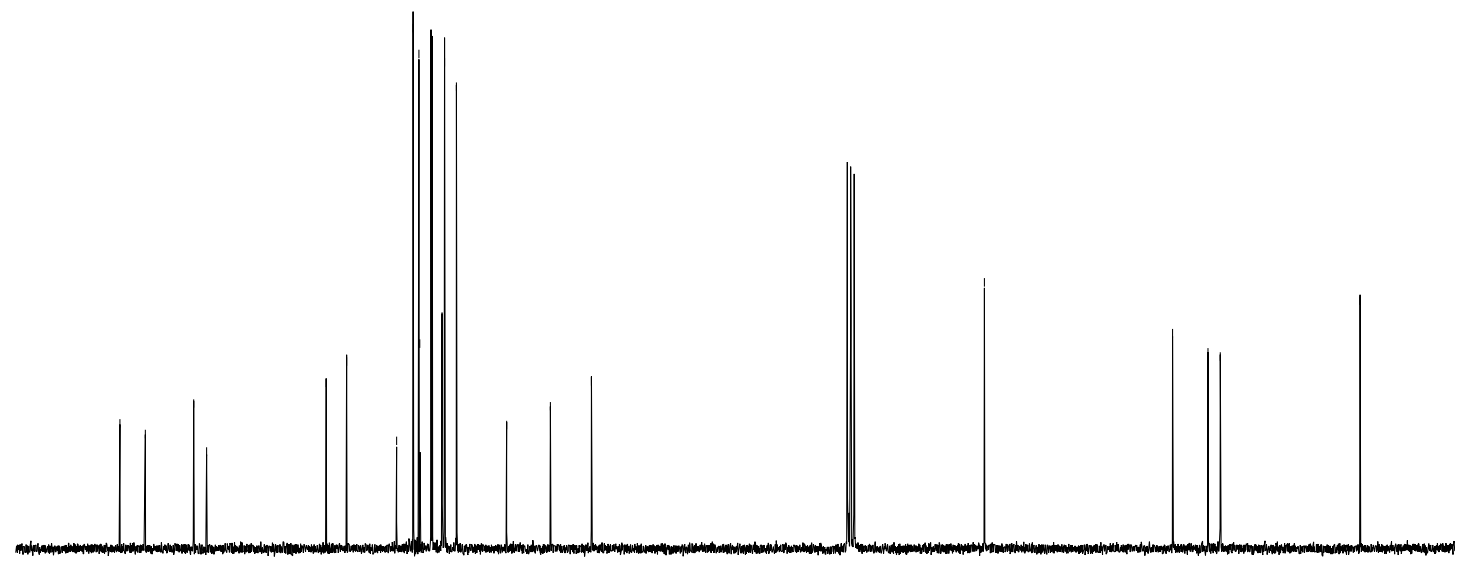

80

170

\section{f1 $(\mathrm{ppm})$}


<smiles>CCOC(=O)C(Cc1cccc(C(F)(F)F)c1)=C1CC(c2ccccc2)c2sc(-c3ccccc3)nc2O1</smiles>

$30 a$
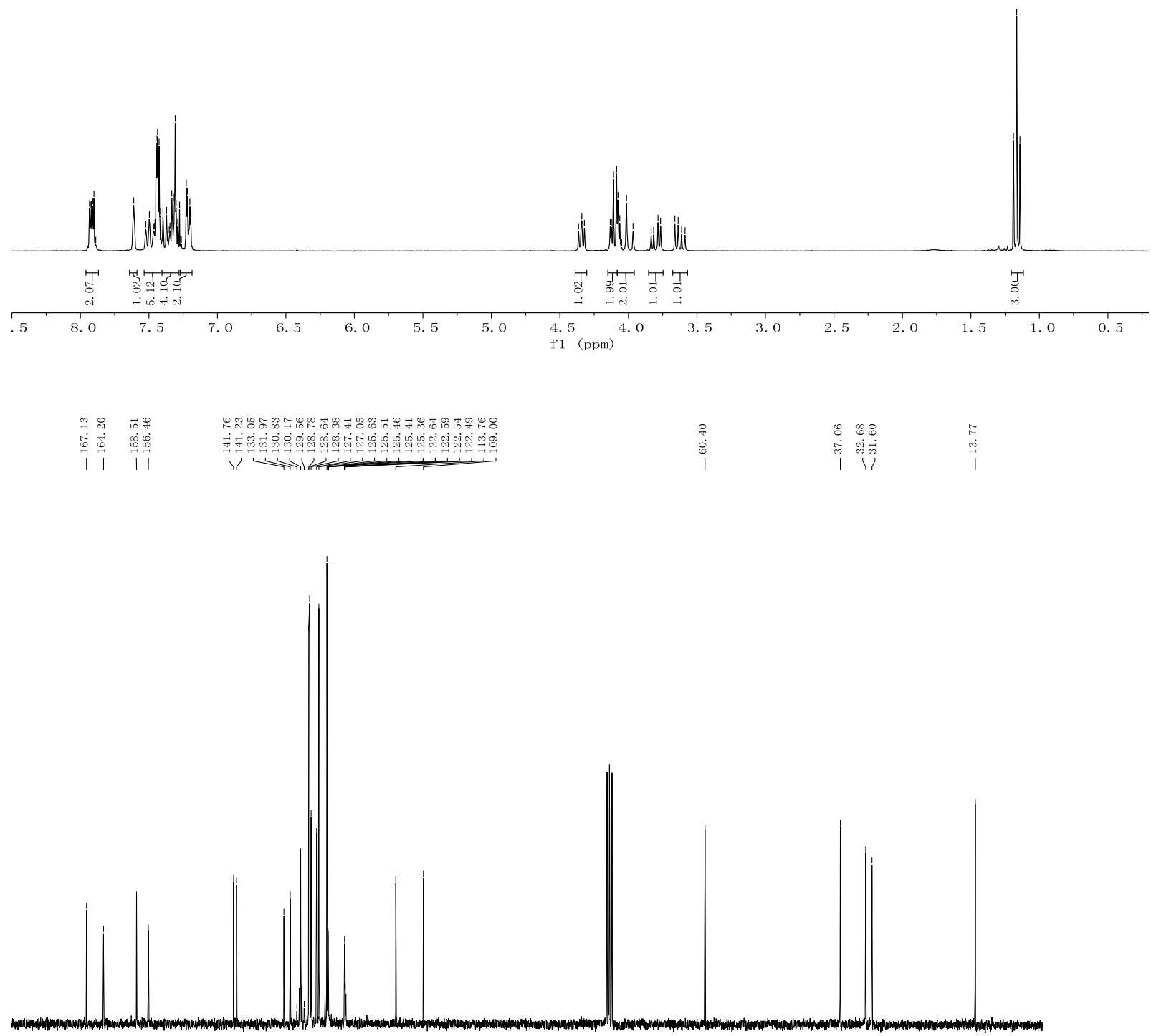

$\begin{array}{llllllllll}80 & 170 & 160 & 150 & 140 & 130 & 120 & 110 & 100 & 90\end{array}$

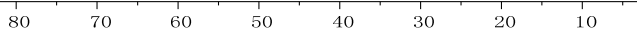




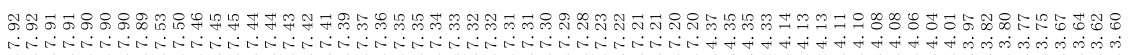

$\stackrel{\infty}{\stackrel{0}{i} \rightarrow-\infty}$
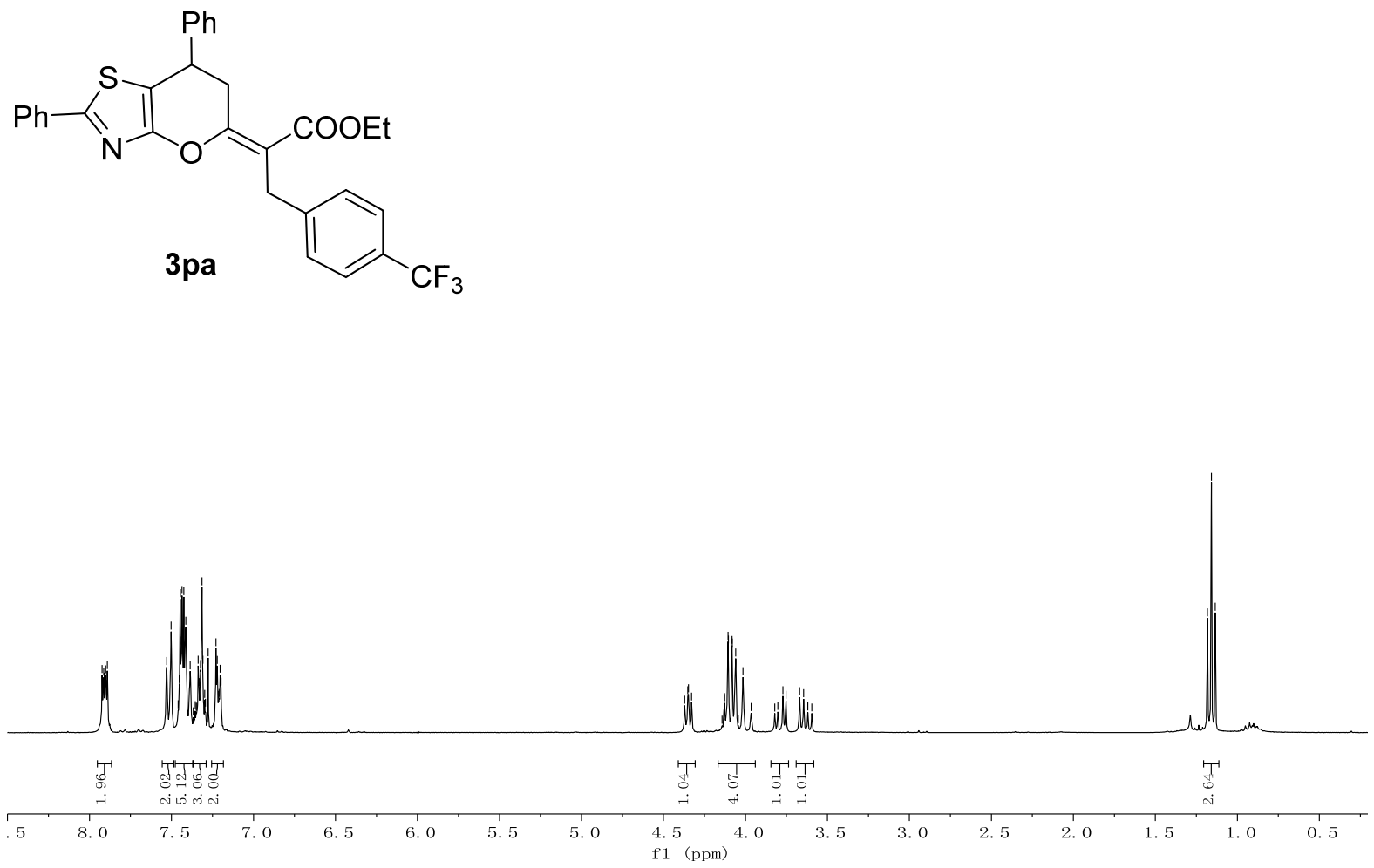

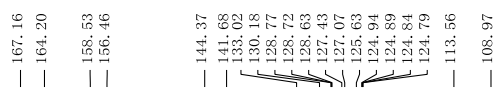

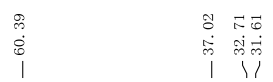

$\stackrel{\vec{\infty}}{\stackrel{\vec{\infty}}{\rightarrow}}$
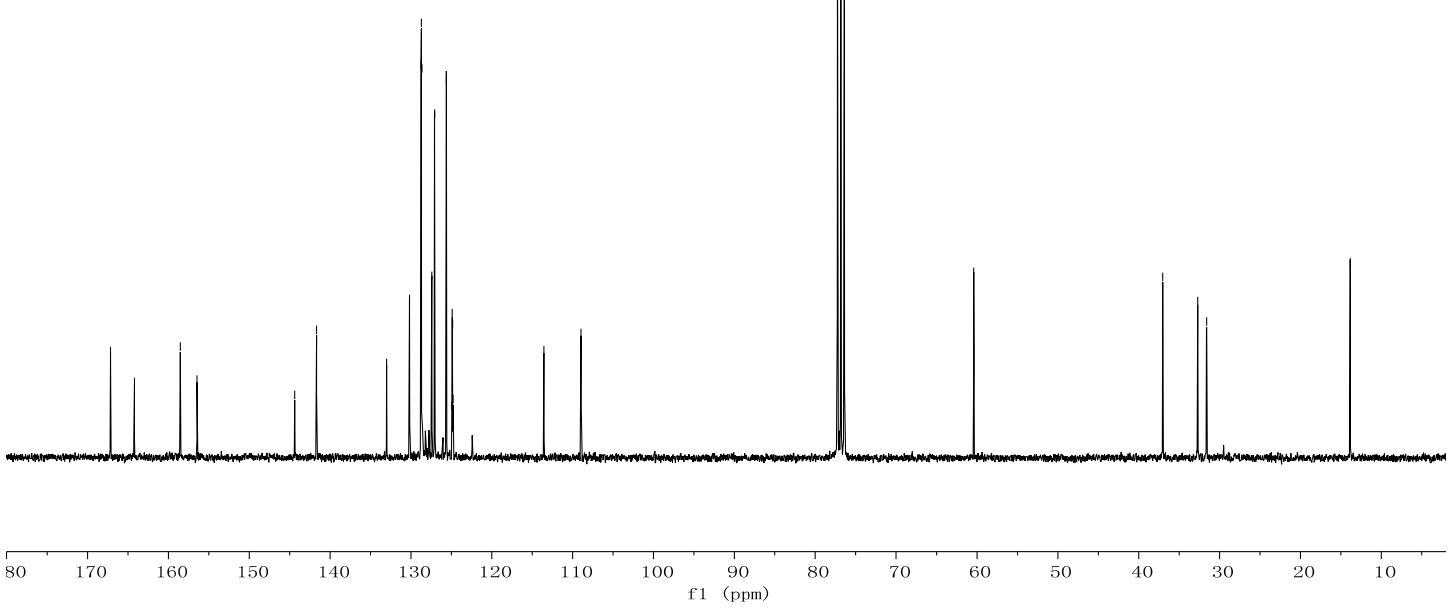

S50 


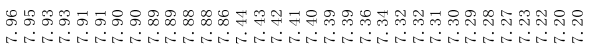

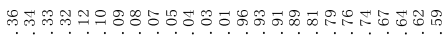

if
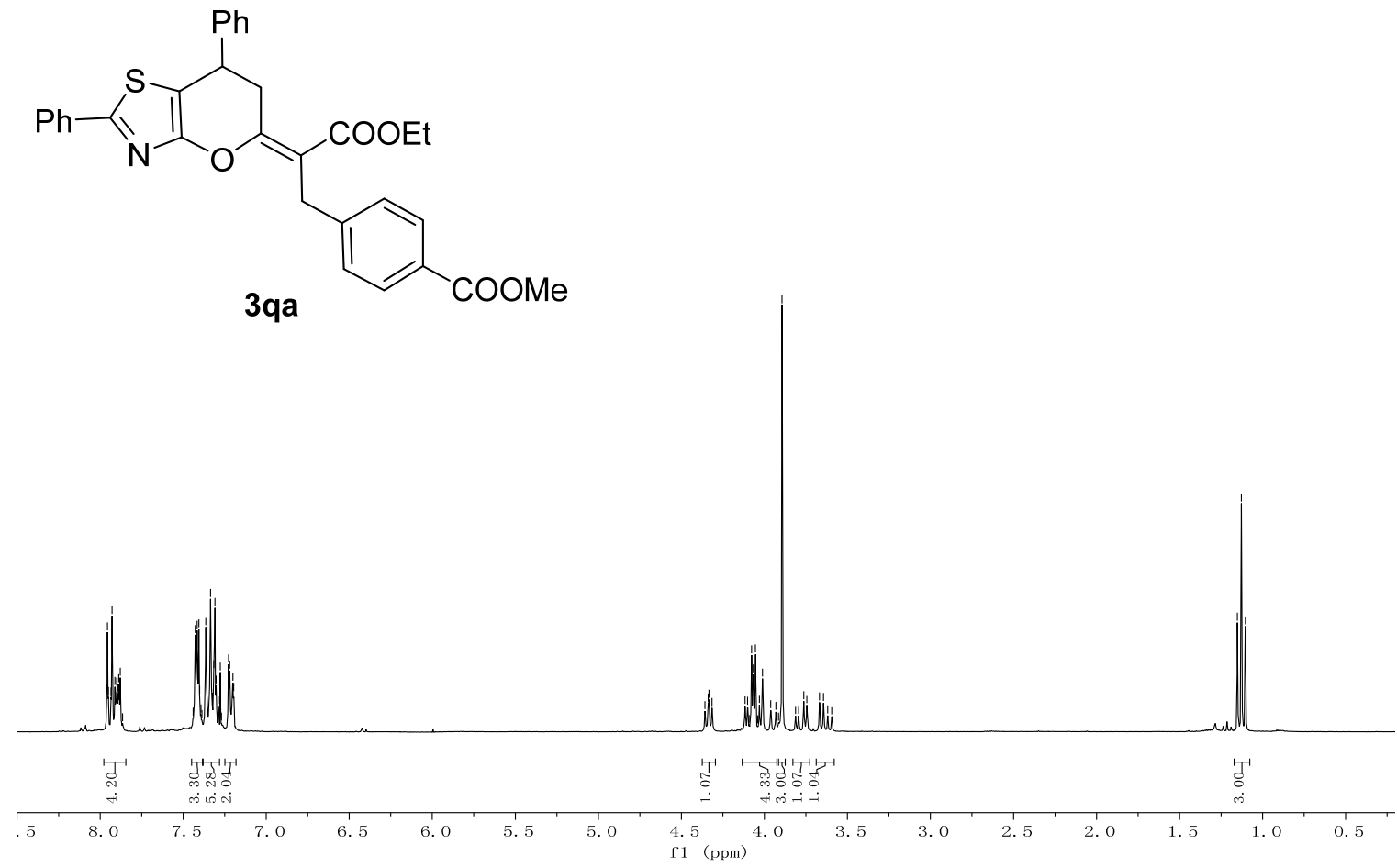

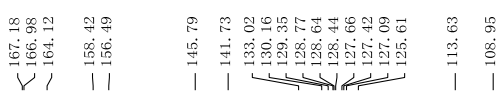

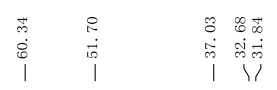

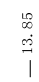
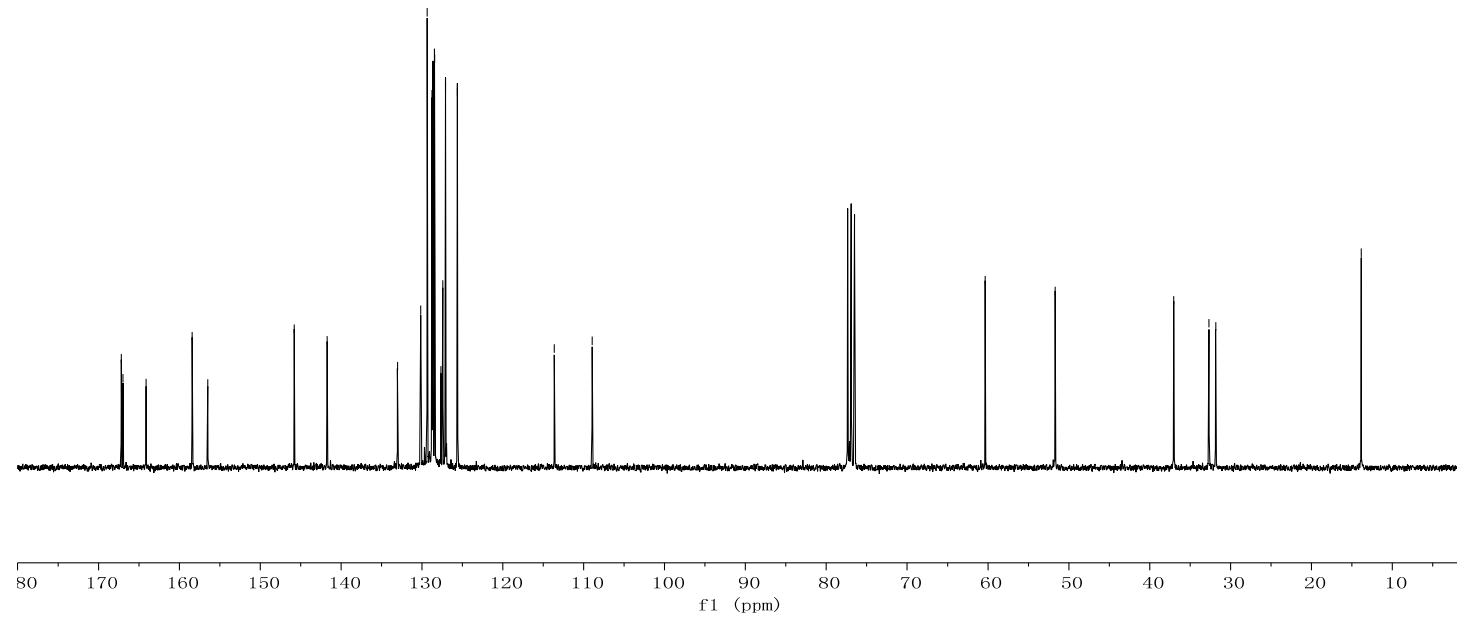

S51 
<smiles>CCOC(=O)C1CC(c2ccccc2)c2sc(-c3ccccc3)nc2O1</smiles>
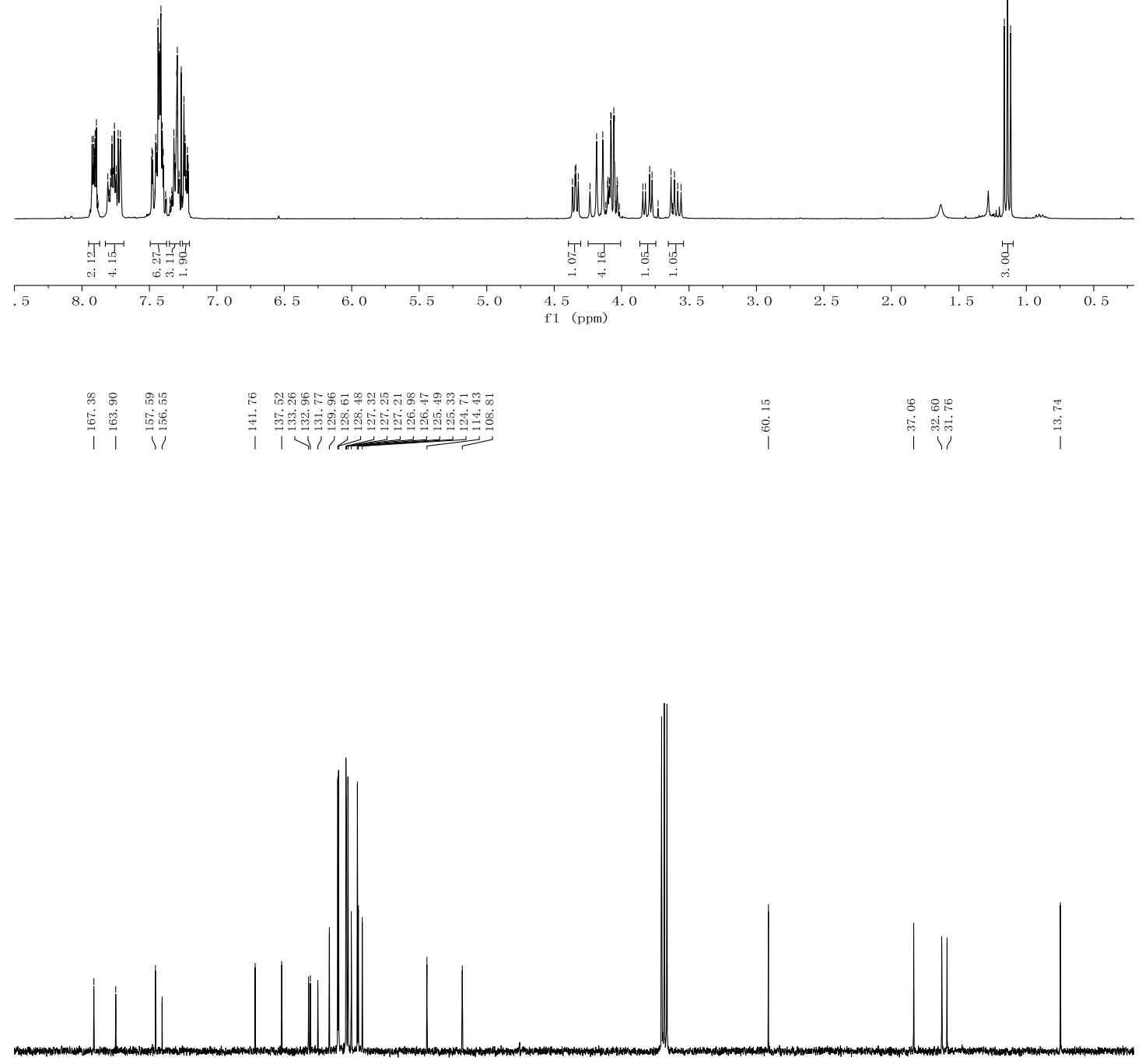

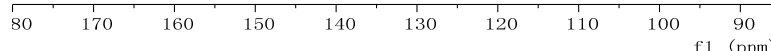


<smiles>OCC(Cc1ccccc1)=C1CC(c2ccccc2)c2sc(-c3ccccc3)nc2O1</smiles>

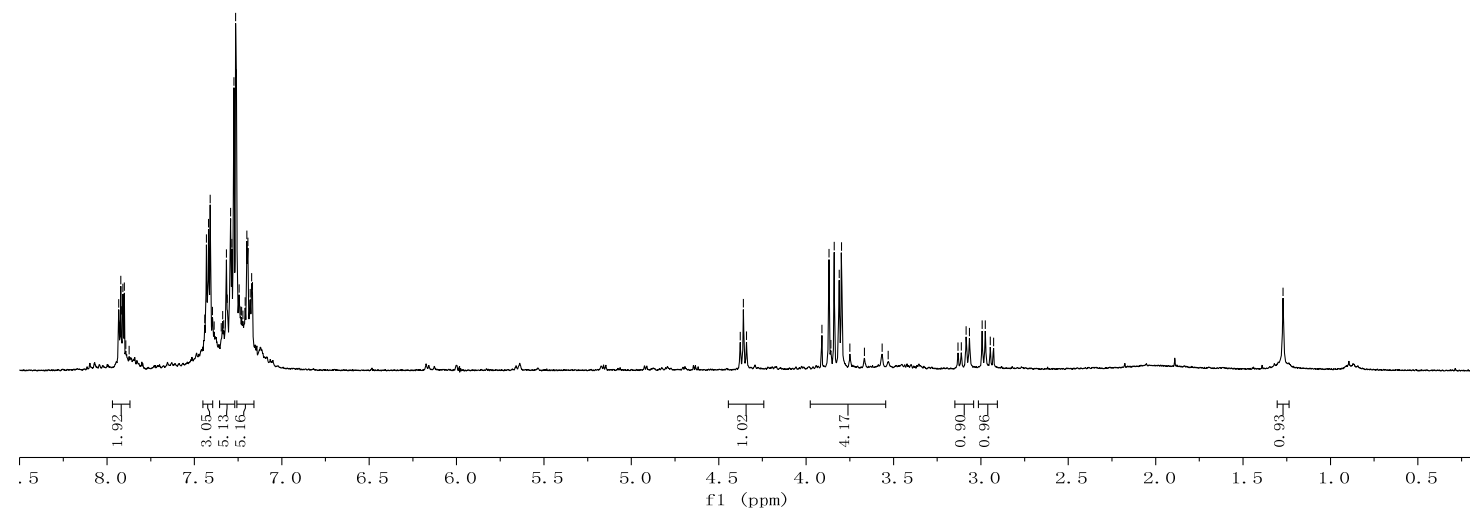

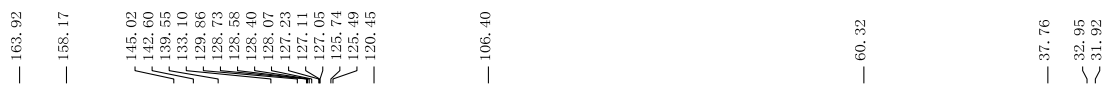

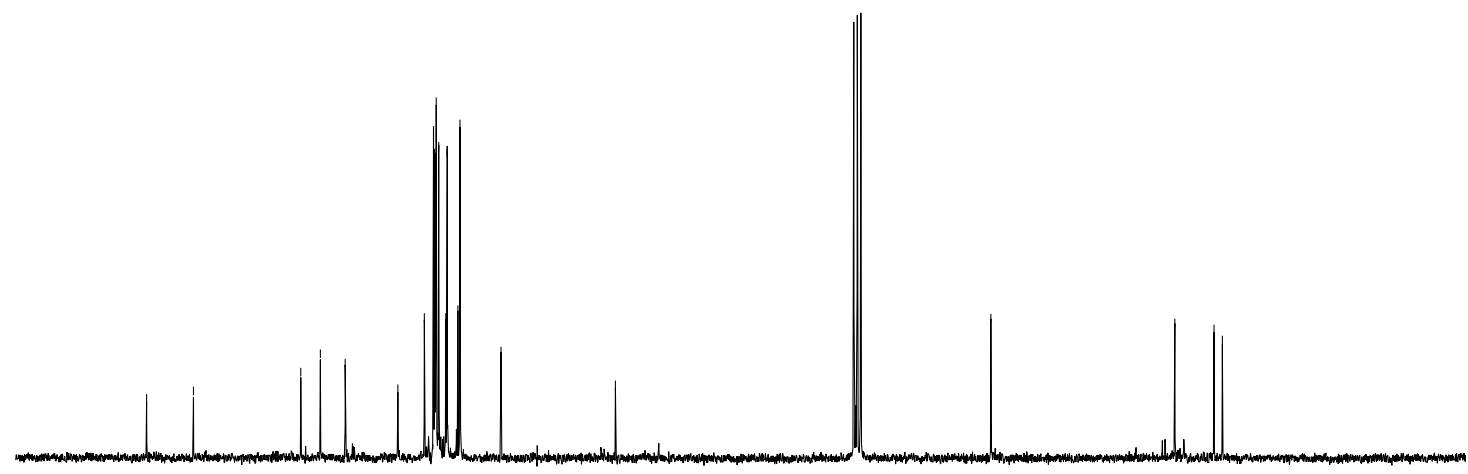

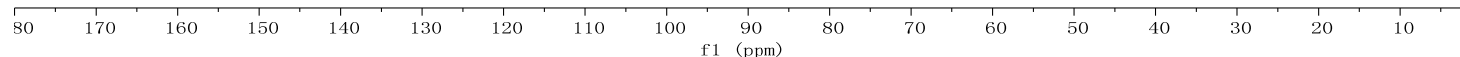


HPLC Chromatograms of the Products

HPLC chromatogram of 3aa

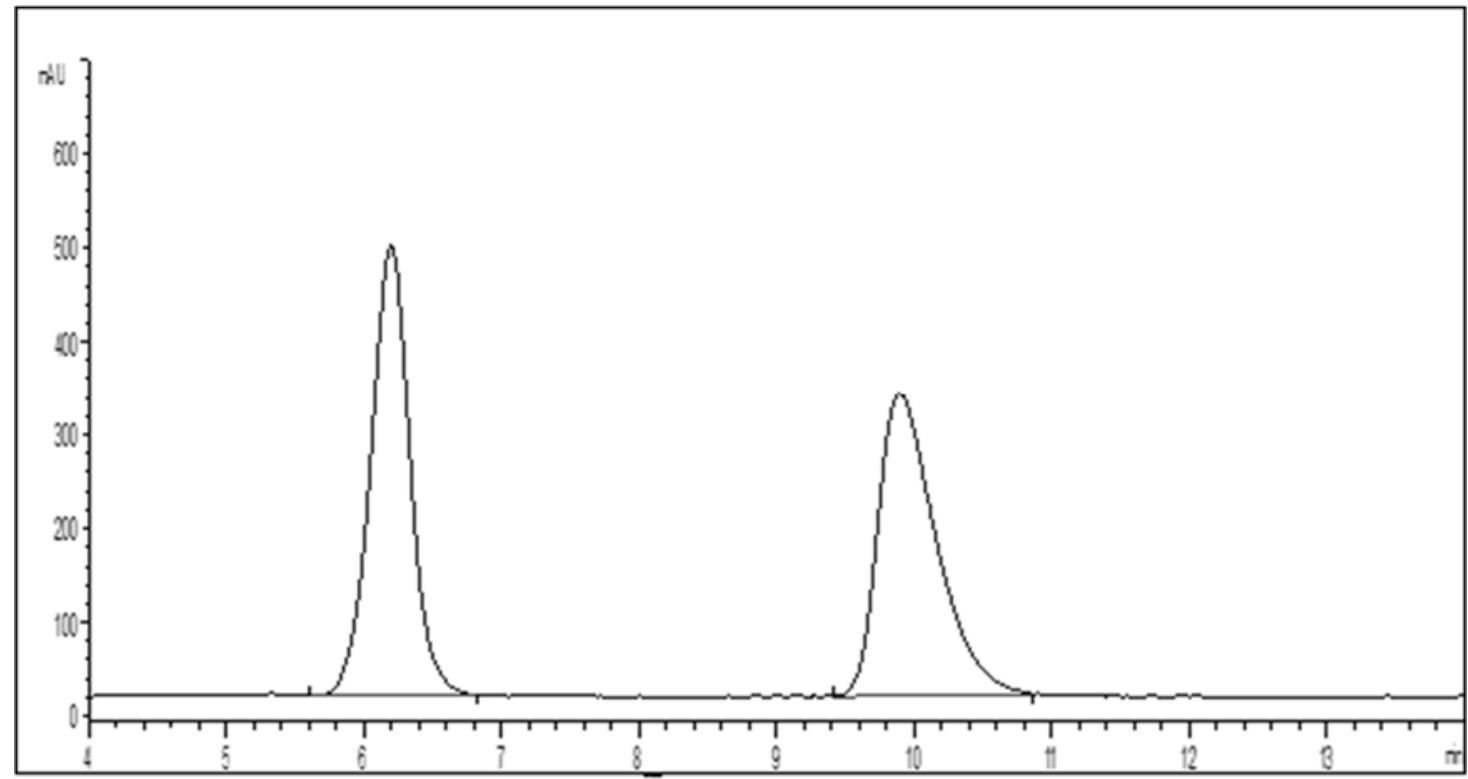

Peak RetTime Type Width Area Height Area

\# [min] [min] $\mathrm{mAU} * \mathrm{~s}[\mathrm{mAU}] \%$

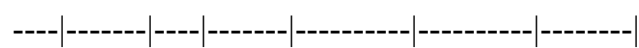

16.200 BB 0.3045 9538.31250 480.3201950.3986

29.896 BB 0.44449387 .45117321 .1958649 .6014

HPLC chromatogram of (+)-3aa

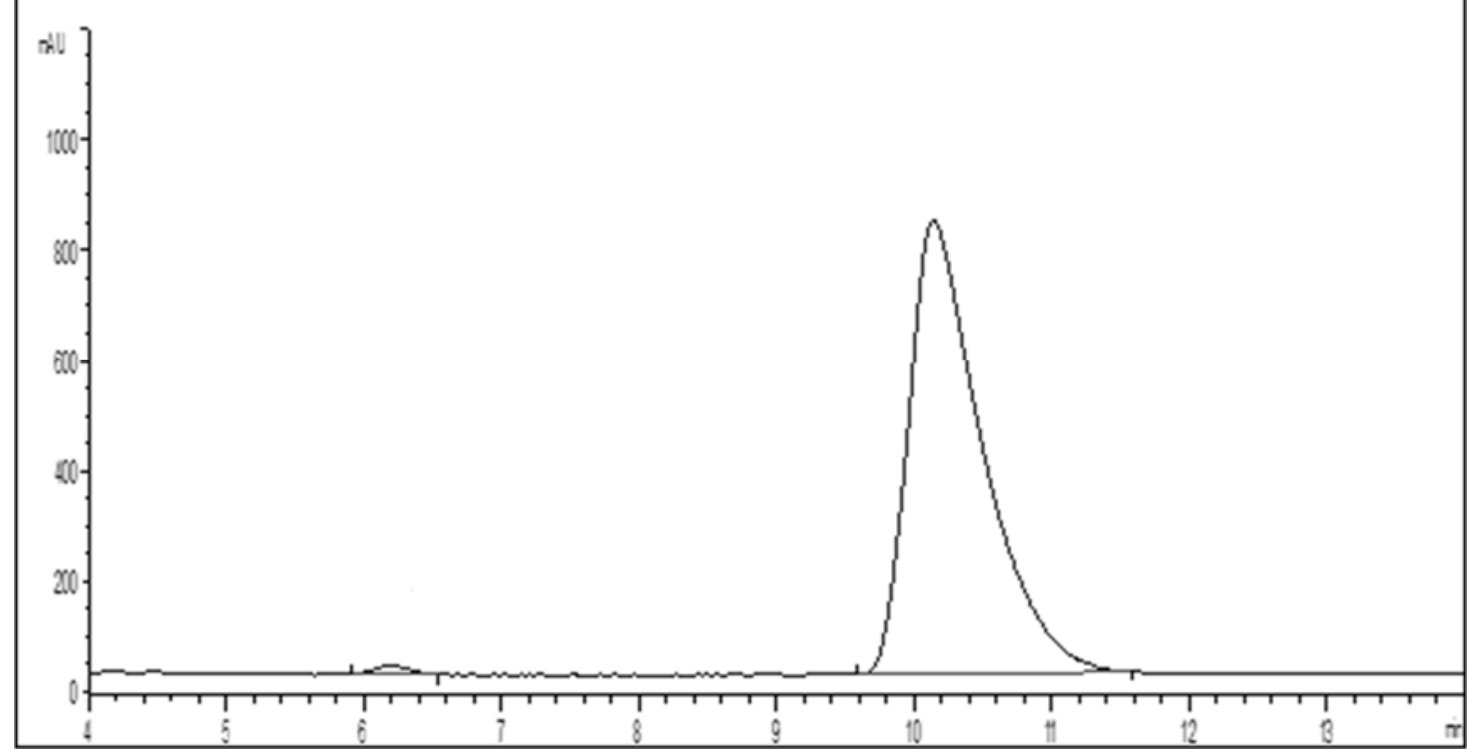

Peak RetTime Type Width Area Height Area

\# [min] [min] $\mathrm{mAU} * \mathrm{~s}[\mathrm{mAU}] \%$

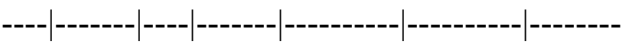

$1 \quad 6.192$ BB 0.2160275 .4563015 .611800 .8925

2 10.140 BB 0.5302 3.05892e4 822.05914 99.1075 
HPLC chromatogram of 3aj

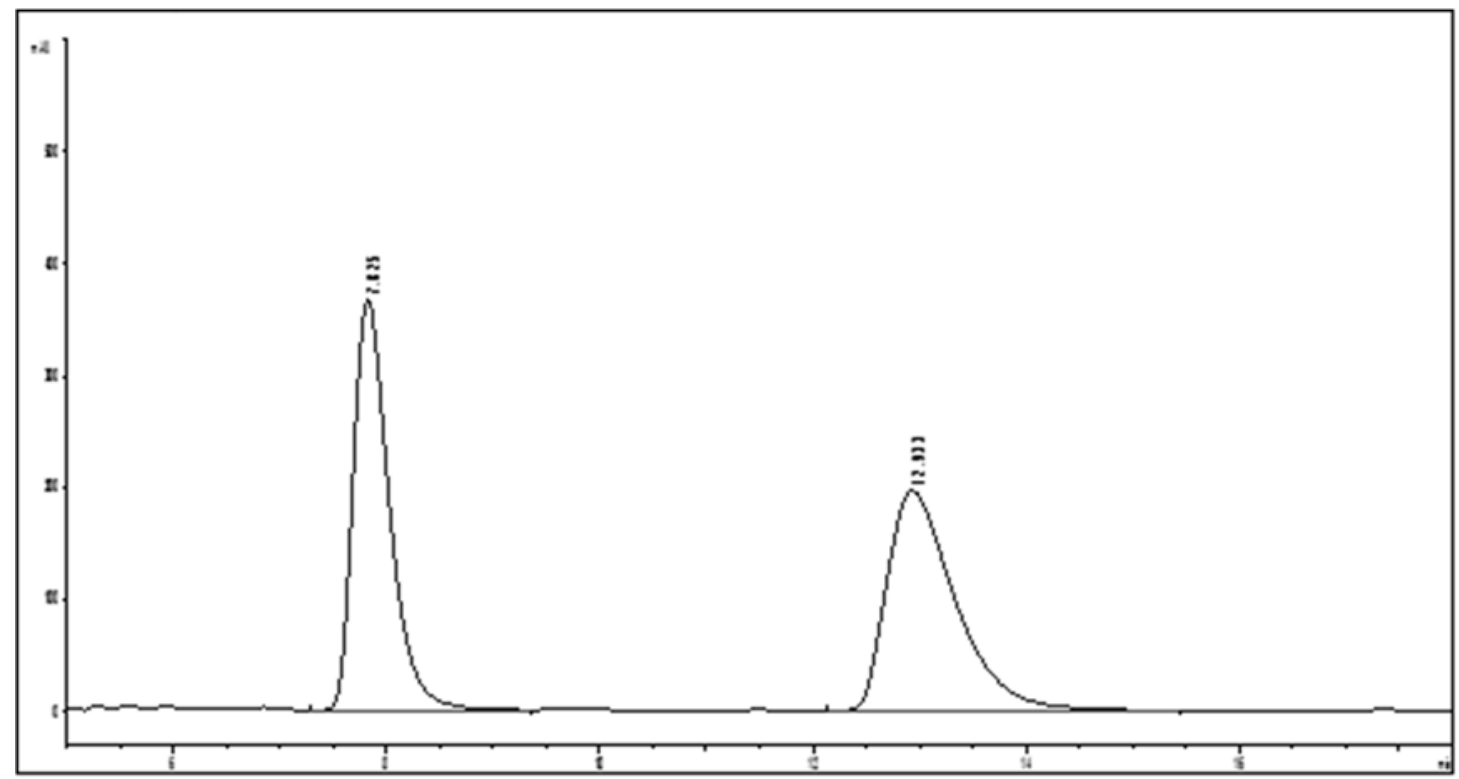

Peak RetTime Type Width Area Height Area

\# [min] [min] $\mathrm{mAU}$ *s [mAU ] \%

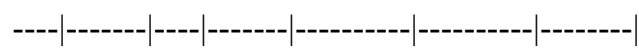

$1 \quad 7.825$ BB 0.36128611 .05566366 .8097249 .7399

212.933 BB 0.68478701 .10840195 .6874850 .2601

\section{HPLC chromatogram of (+)-3aj}

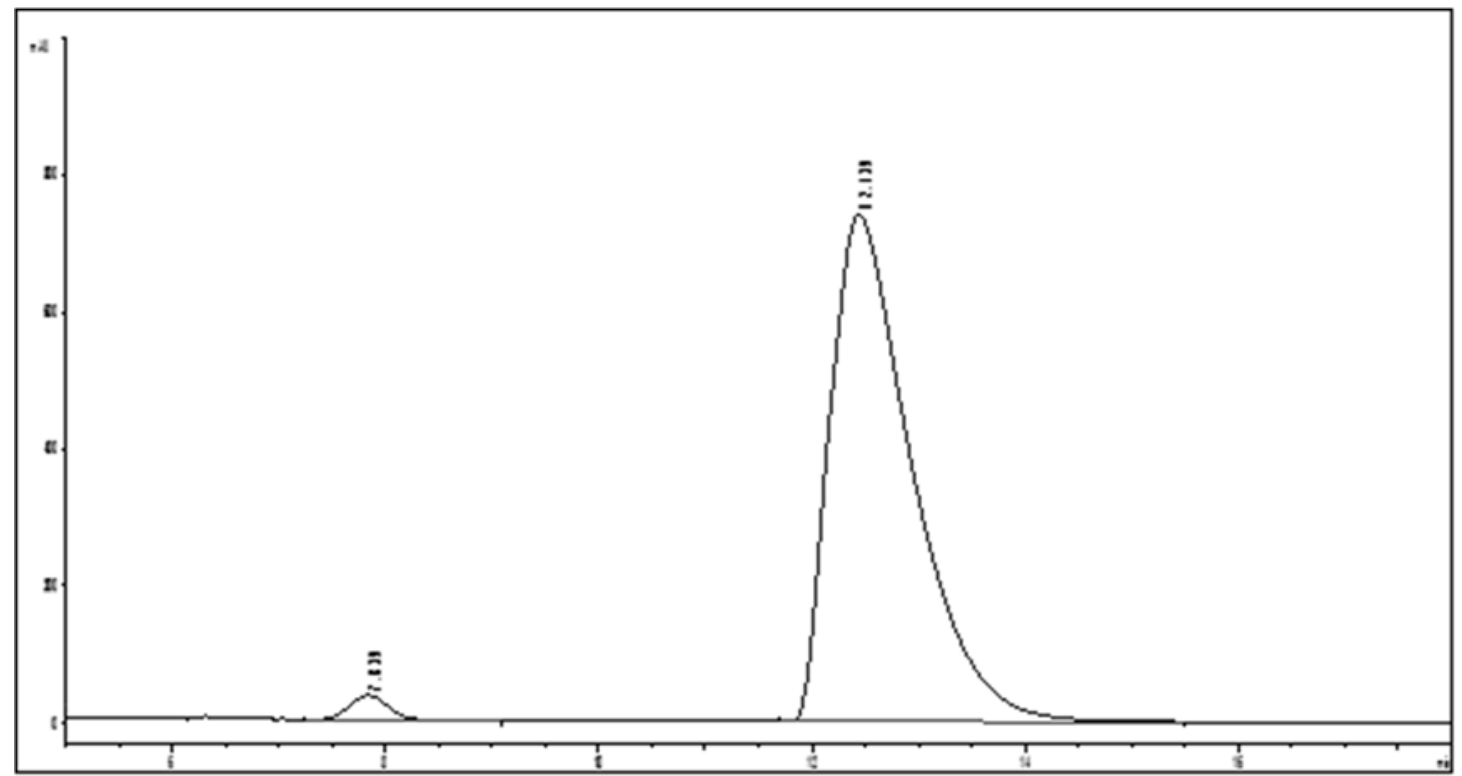

Peak RetTime Type Width Area Height Area

\# [min] [min] $\mathrm{mAU}$ *s [mAU ] \%

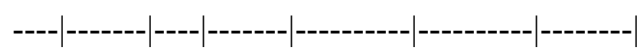

$1 \quad 7.839$ BB 0.42431041 .5711737 .193922 .5775

212.439 BB $0.81123 .93685 \mathrm{e} 4740.2241297 .4225$ 
HPLC chromatogram of 3ma

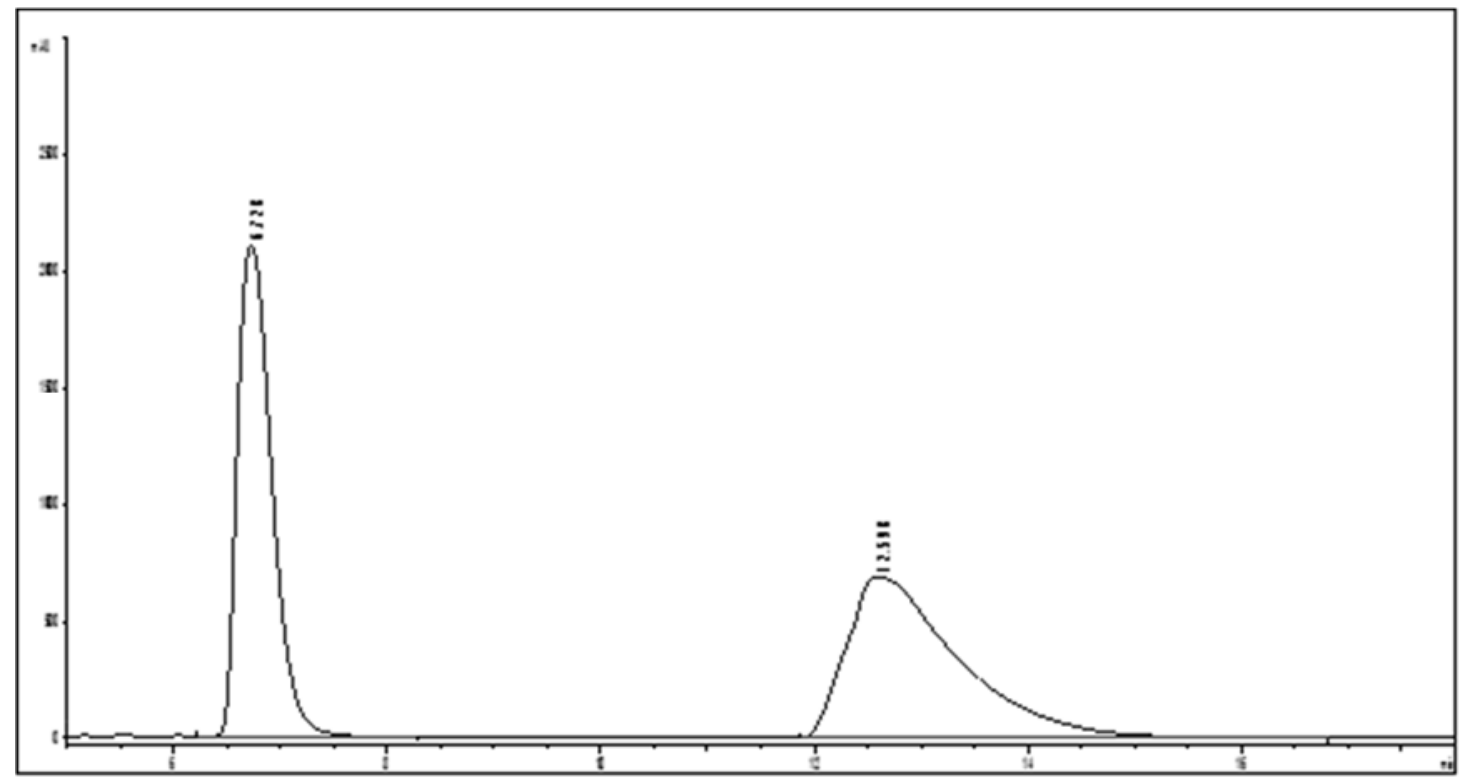

Peak RetTime Type Width Area Height Area

\# [min] [min] mAU *s [mAU ] \%

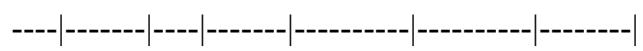

$1 \quad 6.728$ BB $0.35264 .71176 \mathrm{e} 42106.9318848 .6275$

212.598 BB $1.01594 .97774 \mathrm{e} 4685.6520451 .3725$

\section{HPLC chromatogram of (+)-3ma}

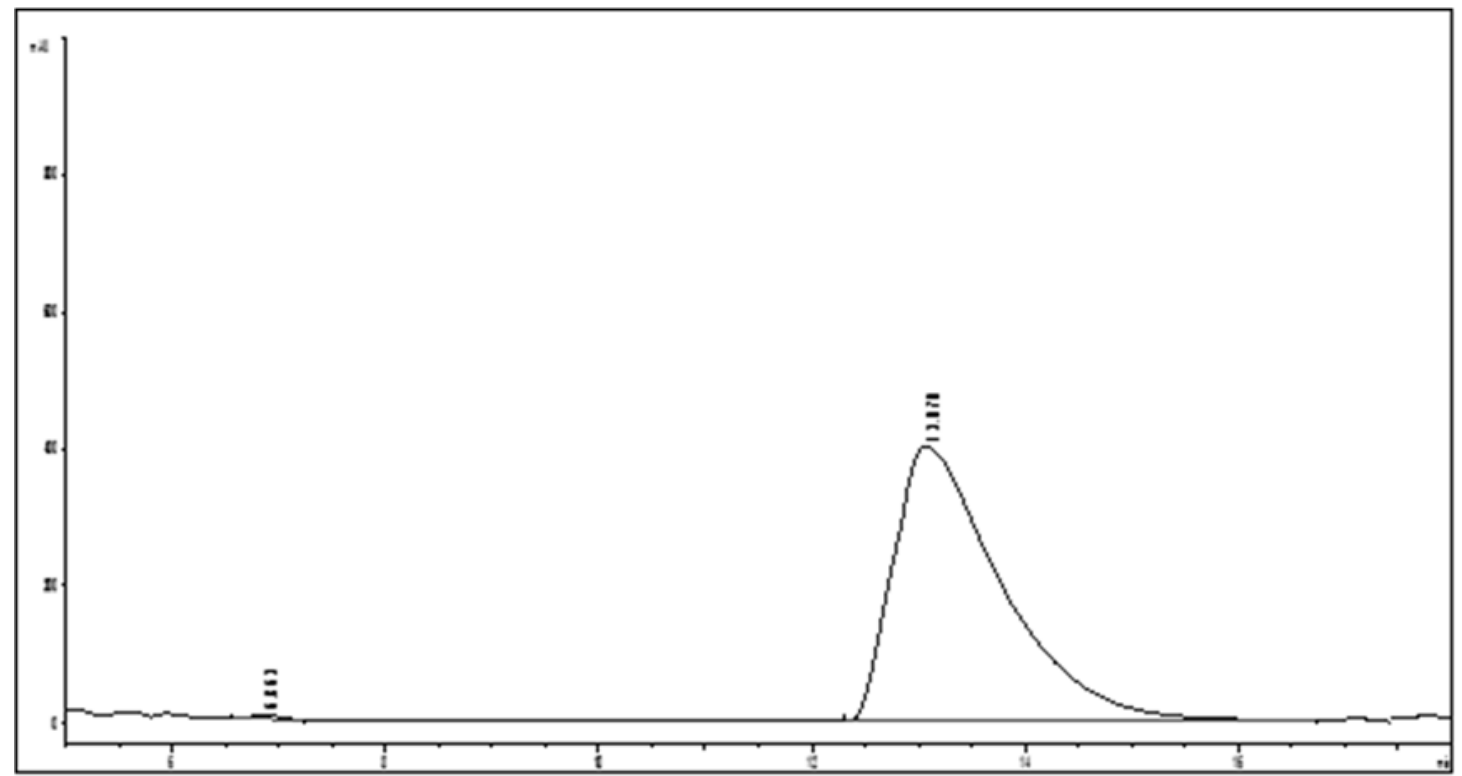

Peak RetTime Type Width Area Height Area

\# [min] [min] mAU *s [mAU ] \%

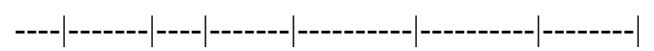

$1 \quad 6.863$ BB 0.2890104 .959595 .626760 .3750

213.070 BB $0.99172 .78845 \mathrm{e} 4397.4952499 .6250$ 
HPLC chromatogram of 3na

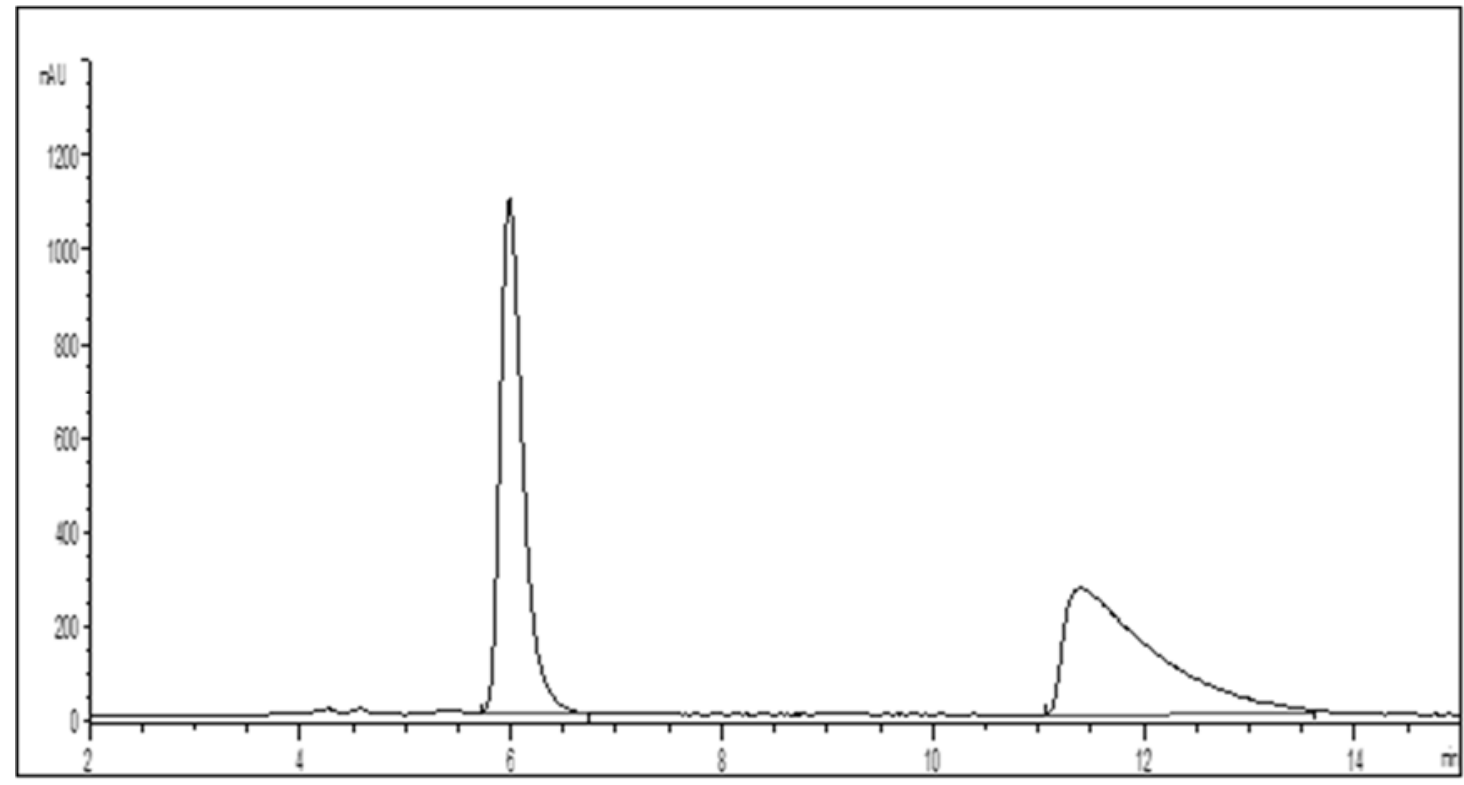

Peak RetTime Type Width Area Height Area

\# [min] [min] mAU *s [mAU ] \%

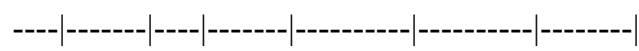

15.985 MM 0.2248 1.62344e4 1089.74548 50.4103

211.403 MM $0.98411 .59701 \mathrm{e} 4270.4637549 .5897$

\section{HPLC chromatogram of (+)-3na}

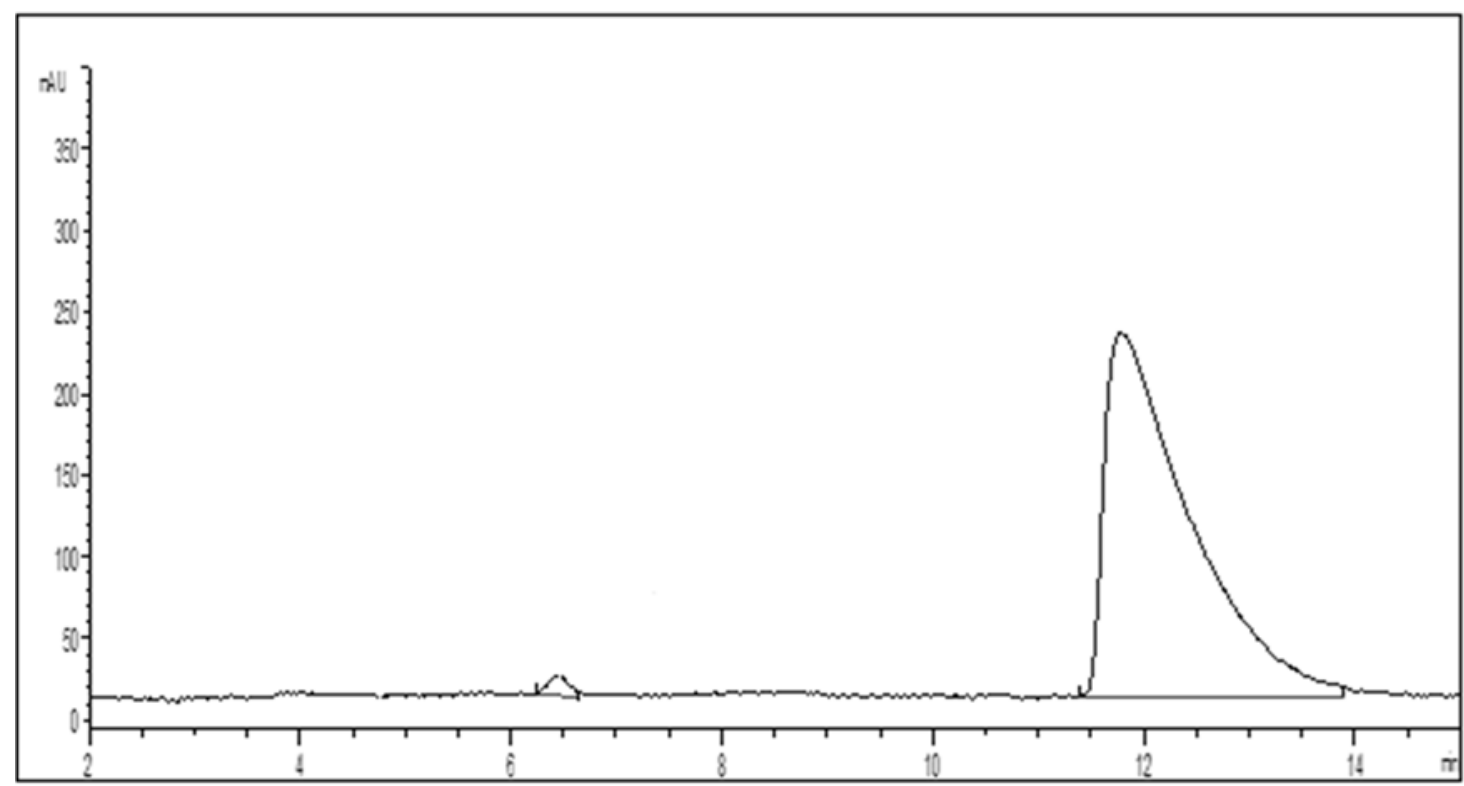

Peak RetTime Type Width Area Height Area

\# [min] [min] mAU *s [mAU ] \%

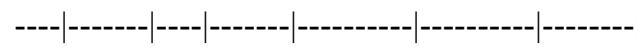

16.455 MM 0.2342175 .9697312 .525221 .3801

2 11.783 MM 0.9345 1.25748e4 224.2615798.6199 


\section{X-Ray Crystallographic Data of 3ma}

Crystallographic data for 3ma have been deposited with the Cambridge Crystallographic Data Centre as deposition number CCDC 1482506.
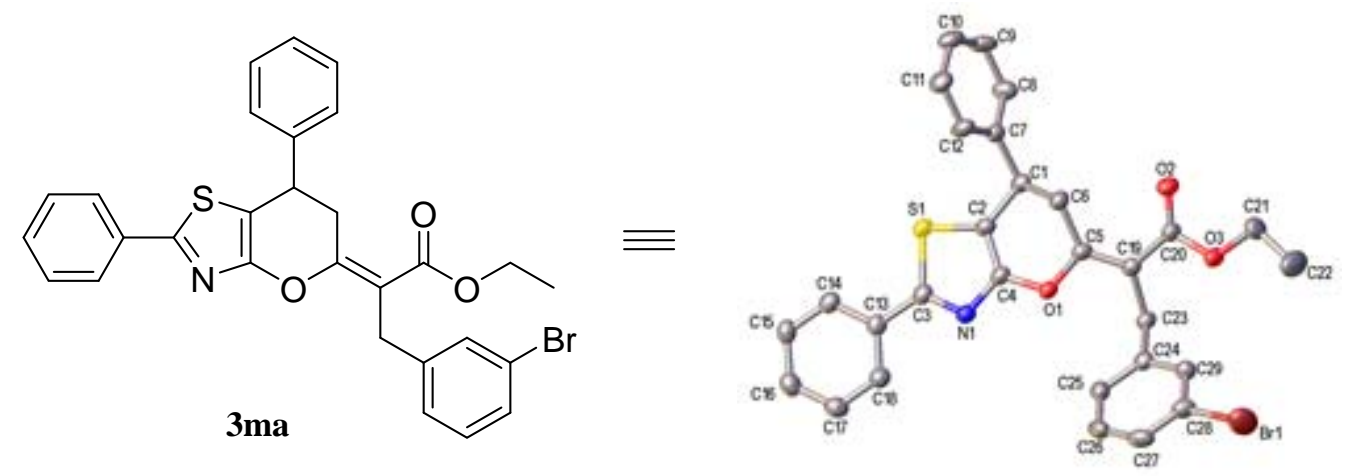

Table 1. Crystal data and structure refinement for 3 ma.

Identification code

Empirical formula

$\mathrm{mx} 3974$

Formula weight

C29 H24 Br N O3 S

Temperature

546.46

Wavelength

$173.1500 \mathrm{~K}$

Crystal system

$0.71073 \AA$

Space group

Unit cell dimensions

Orthorhombic

P 212121

$\begin{array}{ll}\mathrm{a}=5.689(3) \AA & \mathrm{a}=90^{\circ} . \\ \mathrm{b}=20.594(10) \AA & \mathrm{b}=90^{\circ} . \\ \mathrm{c}=21.431(11) \AA & \mathrm{g}=90^{\circ} .\end{array}$

Volume

2511(2) $\AA^{3}$

Z

Density (calculated)

4

Absorption coefficient

$1.446 \mathrm{Mg} / \mathrm{m}^{3}$

$1.752 \mathrm{~mm}^{-1}$

$\mathrm{F}(000)$

Crystal size

Theta range for data collection

1120

$0.26 \times 0.08 \times 0.04 \mathrm{~mm}^{3}$

Index ranges

1.900 to $27.500^{\circ}$.

$-4<=\mathrm{h}<=7,-26<=\mathrm{k}<=26,-26<=1<=27$

Reflections collected

17429

Independent reflections

Completeness to theta $=26.000^{\circ}$

$5777[\mathrm{R}($ int $)=0.0521]$

$99.9 \%$

Absorption correction

Semi-empirical from equivalents

Max. and min. transmission

1.0000 and 0.6724

Refinement method

Full-matrix least-squares on $\mathrm{F}^{2}$

Data / restraints / parameters

5777 / 0 / 317

Goodness-of-fit on $\mathrm{F}^{2}$

1.059

Final R indices [I $>2 \operatorname{sigma}(\mathrm{I})]$

$\mathrm{R} 1=0.0480, \mathrm{wR} 2=0.0987$

$\mathrm{R}$ indices (all data)

$\mathrm{R} 1=0.0542, \mathrm{wR} 2=0.1029$

Absolute structure parameter

$-0.008(6)$

Extinction coefficient

$\mathrm{n} / \mathrm{a}$

Largest diff. peak and hole

0.475 and -0.497 e. $\AA^{-3}$ 
Table 2. Atomic coordinates $\left(\times 10^{4}\right)$ and equivalent isotropic displacement parameters $\left(\AA^{2} \times 10^{3}\right)$ for 3ma. $U(e q)$ is defined as one third of the trace of the orthogonalized $\mathrm{Uij}^{\mathrm{ij}}$ tensor.

\begin{tabular}{|c|c|c|c|c|}
\hline & $\mathrm{x}$ & $\mathrm{y}$ & $\mathrm{z}$ & $\mathrm{U}(\mathrm{eq})$ \\
\hline $\mathrm{Br} 1$ & $818(1)$ & $7277(1)$ & 1894(1) & $60(1)$ \\
\hline S1 & 8783(2) & $4387(1)$ & $5675(1)$ & $32(1)$ \\
\hline $\mathrm{O} 1$ & 4902(5) & 5192(1) & $4433(1)$ & $34(1)$ \\
\hline $\mathrm{O} 2$ & $-24(6)$ & $6614(2)$ & $5110(2)$ & $46(1)$ \\
\hline $\mathrm{O} 3$ & $-1289(6)$ & $6596(2)$ & $4129(1)$ & $42(1)$ \\
\hline N1 & $8075(6)$ & $4483(2)$ & $4493(2)$ & $29(1)$ \\
\hline $\mathrm{C} 1$ & 4874(7) & $5291(2)$ & $5792(2)$ & $30(1)$ \\
\hline $\mathrm{C} 2$ & 6553(7) & $4888(2)$ & $5429(2)$ & $30(1)$ \\
\hline $\mathrm{C} 3$ & $9425(7)$ & $4197(2)$ & $4903(2)$ & $29(1)$ \\
\hline $\mathrm{C} 4$ & $6487(7)$ & $4865(2)$ & $4799(2)$ & $29(1)$ \\
\hline C5 & $3476(7)$ & $5651(2)$ & $4727(2)$ & $30(1)$ \\
\hline C6 & $4215(8)$ & $5864(2)$ & $5364(2)$ & $33(1)$ \\
\hline C7 & $5761(8)$ & $5517(2)$ & $6422(2)$ & $30(1)$ \\
\hline $\mathrm{C} 8$ & $4467(8)$ & $5386(2)$ & $6954(2)$ & $37(1)$ \\
\hline C9 & $5229(10)$ & $5598(2)$ & $7533(2)$ & $44(1)$ \\
\hline $\mathrm{C} 10$ & $7290(10)$ & $5940(2)$ & $7590(2)$ & $42(1)$ \\
\hline $\mathrm{C} 11$ & $8614(9)$ & $6073(2)$ & $7064(2)$ & $41(1)$ \\
\hline $\mathrm{C} 12$ & 7854(8) & $5859(2)$ & $6487(2)$ & $39(1)$ \\
\hline $\mathrm{C} 13$ & 11371(7) & $3762(2)$ & $4732(2)$ & $30(1)$ \\
\hline $\mathrm{C} 14$ & $12953(7)$ & $3526(2)$ & $5176(2)$ & $33(1)$ \\
\hline $\mathrm{C} 15$ & $14795(8)$ & $3123(2)$ & $5004(2)$ & $36(1)$ \\
\hline $\mathrm{C} 16$ & $15094(8)$ & $2964(2)$ & $4383(2)$ & $38(1)$ \\
\hline $\mathrm{C} 17$ & $13567(8)$ & $3195(2)$ & $3939(2)$ & $39(1)$ \\
\hline $\mathrm{C} 18$ & 11692(8) & $3590(2)$ & $4114(2)$ & $34(1)$ \\
\hline $\mathrm{C} 19$ & $1640(7)$ & $5854(2)$ & $4375(2)$ & $28(1)$ \\
\hline $\mathrm{C} 20$ & $81(7)$ & $6383(2)$ & 4591(2) & $30(1)$ \\
\hline $\mathrm{C} 21$ & $-2789(9)$ & $7151(2)$ & $4264(2)$ & $45(1)$ \\
\hline $\mathrm{C} 22$ & $-3724(14)$ & $7405(3)$ & $3681(3)$ & $77(2)$ \\
\hline $\mathrm{C} 23$ & $1155(8)$ & $5542(2)$ & $3745(2)$ & $29(1)$ \\
\hline $\mathrm{C} 24$ & 2381(7) & $5845(2)$ & 3191(2) & $30(1)$ \\
\hline $\mathrm{C} 25$ & $4541(7)$ & $5611(2)$ & $2976(2)$ & $36(1)$ \\
\hline $\mathrm{C} 26$ & $5586(8)$ & $5872(2)$ & $2450(2)$ & $42(1)$ \\
\hline $\mathrm{C} 27$ & 4532(9) & $6379(2)$ & $2129(2)$ & $40(1)$ \\
\hline $\mathrm{C} 28$ & 2402(9) & $6607(2)$ & $2341(2)$ & $37(1)$ \\
\hline $\mathrm{C} 29$ & $1305(8)$ & $6348(2)$ & $2864(2)$ & $34(1)$ \\
\hline
\end{tabular}


Table 3. Bond lengths $[\AA]$ and angles $\left[^{\circ}\right]$ for 3ma.

\begin{tabular}{|c|c|}
\hline Br1-C28 & $1.907(5)$ \\
\hline $\mathrm{S} 1-\mathrm{C} 2$ & $1.718(4)$ \\
\hline S1-C3 & $1.737(4)$ \\
\hline $\mathrm{O} 1-\mathrm{C} 4$ & $1.371(5)$ \\
\hline $\mathrm{O} 1-\mathrm{C} 5$ & $1.395(5)$ \\
\hline $\mathrm{O} 2-\mathrm{C} 20$ & $1.210(5)$ \\
\hline $\mathrm{O} 3-\mathrm{C} 20$ & $1.335(5)$ \\
\hline O3-C21 & $1.454(5)$ \\
\hline N1-C3 & $1.308(5)$ \\
\hline N1-C4 & $1.366(5)$ \\
\hline C1-H1 & 0.9800 \\
\hline $\mathrm{C} 1-\mathrm{C} 2$ & $1.487(6)$ \\
\hline $\mathrm{C} 1-\mathrm{C} 6$ & $1.541(6)$ \\
\hline $\mathrm{C} 1-\mathrm{C} 7$ & $1.515(5)$ \\
\hline $\mathrm{C} 2-\mathrm{C} 4$ & $1.351(6)$ \\
\hline $\mathrm{C} 3-\mathrm{C} 13$ & $1.470(6)$ \\
\hline $\mathrm{C} 5-\mathrm{C} 6$ & $1.495(6)$ \\
\hline C5-C19 & $1.355(6)$ \\
\hline C6-H6A & 0.9700 \\
\hline C6-H6B & 0.9700 \\
\hline C7-C8 & $1.383(6)$ \\
\hline $\mathrm{C} 7-\mathrm{C} 12$ & $1.389(6)$ \\
\hline C8-H8 & 0.9300 \\
\hline C8-C9 & $1.387(6)$ \\
\hline C9-H9 & 0.9300 \\
\hline C9-C10 & $1.372(7)$ \\
\hline C10-H10 & 0.9300 \\
\hline C10-C11 & $1.384(7)$ \\
\hline C11-H11 & 0.9300 \\
\hline C11-C12 & $1.383(6)$ \\
\hline C12-H12 & 0.9300 \\
\hline C13-C14 & $1.396(6)$ \\
\hline C13-C18 & $1.384(6)$ \\
\hline C14-H14 & 0.9300 \\
\hline C14-C15 & $1.386(6)$ \\
\hline C15-H15 & 0.9300 \\
\hline $\mathrm{C} 15-\mathrm{C} 16$ & $1.381(6)$ \\
\hline C16-H16 & 0.9300 \\
\hline $\mathrm{C} 16-\mathrm{C} 17$ & $1.374(6)$ \\
\hline C17-H17 & 0.9300 \\
\hline $\mathrm{C} 17-\mathrm{C} 18$ & $1.393(6)$ \\
\hline C18-H18 & 0.9300 \\
\hline
\end{tabular}




\begin{tabular}{|c|c|}
\hline C19-C20 & $1.479(6)$ \\
\hline C19-C23 & $1.520(5)$ \\
\hline $\mathrm{C} 21-\mathrm{H} 21 \mathrm{~A}$ & 0.9700 \\
\hline C21-H21B & 0.9700 \\
\hline $\mathrm{C} 21-\mathrm{C} 22$ & $1.454(7)$ \\
\hline $\mathrm{C} 22-\mathrm{H} 22 \mathrm{~A}$ & 0.9600 \\
\hline C22-H22B & 0.9600 \\
\hline $\mathrm{C} 22-\mathrm{H} 22 \mathrm{C}$ & 0.9600 \\
\hline $\mathrm{C} 23-\mathrm{H} 23 \mathrm{~A}$ & 0.9700 \\
\hline C23-H23B & 0.9700 \\
\hline C23-C24 & $1.513(6)$ \\
\hline $\mathrm{C} 24-\mathrm{C} 25$ & $1.398(6)$ \\
\hline C24-C29 & $1.392(6)$ \\
\hline $\mathrm{C} 25-\mathrm{H} 25$ & 0.9300 \\
\hline $\mathrm{C} 25-\mathrm{C} 26$ & $1.384(6)$ \\
\hline $\mathrm{C} 26-\mathrm{H} 26$ & 0.9300 \\
\hline C26-C27 & $1.385(7)$ \\
\hline C27-H27 & 0.9300 \\
\hline $\mathrm{C} 27-\mathrm{C} 28$ & $1.377(7)$ \\
\hline C28-C29 & $1.389(6)$ \\
\hline C29-H29 & 0.9300 \\
\hline C2-S1-C3 & $89.9(2)$ \\
\hline $\mathrm{C} 4-\mathrm{O} 1-\mathrm{C} 5$ & $117.2(3)$ \\
\hline $\mathrm{C} 20-\mathrm{O} 3-\mathrm{C} 21$ & $116.9(3)$ \\
\hline C3-N1-C4 & $109.0(3)$ \\
\hline C2-C1-H1 & 107.8 \\
\hline C2-C1-C6 & $105.8(3)$ \\
\hline $\mathrm{C} 2-\mathrm{C} 1-\mathrm{C} 7$ & 115.1(4) \\
\hline C6-C1-H1 & 107.8 \\
\hline C7-C1-H1 & 107.8 \\
\hline C7-C1-C6 & 112.1(3) \\
\hline C1-C2-S1 & $130.5(3)$ \\
\hline C4-C2-S1 & $107.8(3)$ \\
\hline $\mathrm{C} 4-\mathrm{C} 2-\mathrm{C} 1$ & $121.6(4)$ \\
\hline N1-C3-S1 & $114.5(3)$ \\
\hline N1-C3-C13 & $123.3(4)$ \\
\hline C13-C3-S1 & $122.2(3)$ \\
\hline N1-C4-O1 & 116.3(3) \\
\hline C2-C4-O1 & $124.9(4)$ \\
\hline C2-C4-N1 & $118.7(4)$ \\
\hline $\mathrm{O} 1-\mathrm{C} 5-\mathrm{C} 6$ & $116.6(3)$ \\
\hline $\mathrm{C} 19-\mathrm{C} 5-\mathrm{O} 1$ & $114.0(4)$ \\
\hline $\mathrm{C} 19-\mathrm{C} 5-\mathrm{C} 6$ & $129.4(4)$ \\
\hline
\end{tabular}




\begin{tabular}{|c|c|}
\hline C1-C6-H6A & 109.0 \\
\hline C1-C6-H6B & 109.0 \\
\hline $\mathrm{C} 5-\mathrm{C} 6-\mathrm{C} 1$ & $112.8(3)$ \\
\hline C5-C6-H6A & 109.0 \\
\hline C5-C6-H6B & 109.0 \\
\hline H6A-C6-H6B & 107.8 \\
\hline $\mathrm{C} 8-\mathrm{C} 7-\mathrm{C} 1$ & $119.8(4)$ \\
\hline $\mathrm{C} 8-\mathrm{C} 7-\mathrm{C} 12$ & $118.3(4)$ \\
\hline $\mathrm{C} 12-\mathrm{C} 7-\mathrm{C} 1$ & $122.0(4)$ \\
\hline C7-C8-H8 & 119.7 \\
\hline C7-C8-C9 & $120.6(4)$ \\
\hline C9-C8-H8 & 119.7 \\
\hline C8-C9-H9 & 119.7 \\
\hline $\mathrm{C} 10-\mathrm{C} 9-\mathrm{C} 8$ & $120.5(4)$ \\
\hline C10-C9-H9 & 119.7 \\
\hline C9-C10-H10 & 120.2 \\
\hline C9-C10-C11 & $119.6(4)$ \\
\hline C11-C10-H10 & 120.2 \\
\hline C10-C11-H11 & 120.1 \\
\hline $\mathrm{C} 12-\mathrm{C} 11-\mathrm{C} 10$ & $119.7(4)$ \\
\hline $\mathrm{C} 12-\mathrm{C} 11-\mathrm{H} 11$ & 120.1 \\
\hline C7-C12-H12 & 119.4 \\
\hline $\mathrm{C} 11-\mathrm{C} 12-\mathrm{C} 7$ & $121.2(4)$ \\
\hline C11-C12-H12 & 119.4 \\
\hline $\mathrm{C} 14-\mathrm{C} 13-\mathrm{C} 3$ & $121.8(4)$ \\
\hline $\mathrm{C} 18-\mathrm{C} 13-\mathrm{C} 3$ & $119.6(4)$ \\
\hline $\mathrm{C} 18-\mathrm{C} 13-\mathrm{C} 14$ & $118.6(4)$ \\
\hline C13-C14-H14 & 119.5 \\
\hline C15-C14-C13 & $121.0(4)$ \\
\hline C15-C14-H14 & 119.5 \\
\hline C14-C15-H15 & 120.3 \\
\hline C16-C15-C14 & $119.3(4)$ \\
\hline C16-C15-H15 & 120.3 \\
\hline C15-C16-H16 & 119.7 \\
\hline C17-C16-C15 & $120.5(4)$ \\
\hline C17-C16-H16 & 119.7 \\
\hline C16-C17-H17 & 120.0 \\
\hline C16-C17-C18 & $120.0(4)$ \\
\hline C18-C17-H17 & 120.0 \\
\hline C13-C18-C17 & $120.5(4)$ \\
\hline C13-C18-H18 & 119.7 \\
\hline C17-C18-H18 & 119.7 \\
\hline C5-C19-C20 & $121.1(4)$ \\
\hline C5-C19-C23 & $120.2(4)$ \\
\hline
\end{tabular}




\begin{tabular}{|c|c|}
\hline $\mathrm{C} 20-\mathrm{C} 19-\mathrm{C} 23$ & $118.7(4)$ \\
\hline $\mathrm{O} 2-\mathrm{C} 20-\mathrm{O} 3$ & $121.6(4)$ \\
\hline O2-C20-C19 & $127.3(4)$ \\
\hline O3-C20-C19 & $111.1(4)$ \\
\hline $\mathrm{O} 3-\mathrm{C} 21-\mathrm{H} 21 \mathrm{~A}$ & 109.9 \\
\hline $\mathrm{O} 3-\mathrm{C} 21-\mathrm{H} 21 \mathrm{~B}$ & 109.9 \\
\hline $\mathrm{O} 3-\mathrm{C} 21-\mathrm{C} 22$ & $109.0(4)$ \\
\hline $\mathrm{H} 21 \mathrm{~A}-\mathrm{C} 21-\mathrm{H} 21 \mathrm{~B}$ & 108.3 \\
\hline $\mathrm{C} 22-\mathrm{C} 21-\mathrm{H} 21 \mathrm{~A}$ & 109.9 \\
\hline $\mathrm{C} 22-\mathrm{C} 21-\mathrm{H} 21 \mathrm{~B}$ & 109.9 \\
\hline $\mathrm{C} 21-\mathrm{C} 22-\mathrm{H} 22 \mathrm{~A}$ & 109.5 \\
\hline $\mathrm{C} 21-\mathrm{C} 22-\mathrm{H} 22 \mathrm{~B}$ & 109.5 \\
\hline $\mathrm{C} 21-\mathrm{C} 22-\mathrm{H} 22 \mathrm{C}$ & 109.5 \\
\hline $\mathrm{H} 22 \mathrm{~A}-\mathrm{C} 22-\mathrm{H} 22 \mathrm{~B}$ & 109.5 \\
\hline $\mathrm{H} 22 \mathrm{~A}-\mathrm{C} 22-\mathrm{H} 22 \mathrm{C}$ & 109.5 \\
\hline $\mathrm{H} 22 \mathrm{~B}-\mathrm{C} 22-\mathrm{H} 22 \mathrm{C}$ & 109.5 \\
\hline $\mathrm{C} 19-\mathrm{C} 23-\mathrm{H} 23 \mathrm{~A}$ & 108.3 \\
\hline C19-C23-H23B & 108.3 \\
\hline $\mathrm{H} 23 \mathrm{~A}-\mathrm{C} 23-\mathrm{H} 23 \mathrm{~B}$ & 107.4 \\
\hline C24-C23-C19 & $116.1(3)$ \\
\hline $\mathrm{C} 24-\mathrm{C} 23-\mathrm{H} 23 \mathrm{~A}$ & 108.3 \\
\hline C24-C23-H23B & 108.3 \\
\hline C25-C24-C23 & $121.5(4)$ \\
\hline C29-C24-C23 & $120.0(4)$ \\
\hline C29-C24-C25 & $118.5(4)$ \\
\hline $\mathrm{C} 24-\mathrm{C} 25-\mathrm{H} 25$ & 119.6 \\
\hline C26-C25-C24 & $120.8(4)$ \\
\hline C26-C25-H25 & 119.6 \\
\hline $\mathrm{C} 25-\mathrm{C} 26-\mathrm{H} 26$ & 119.6 \\
\hline $\mathrm{C} 25-\mathrm{C} 26-\mathrm{C} 27$ & $120.7(4)$ \\
\hline C27-C26-H26 & 119.6 \\
\hline C26-C27-H27 & 120.9 \\
\hline C28-C27-C26 & $118.3(4)$ \\
\hline C28-C27-H27 & 120.9 \\
\hline $\mathrm{C} 27-\mathrm{C} 28-\mathrm{Br} 1$ & $119.8(4)$ \\
\hline C27-C28-C29 & $122.1(4)$ \\
\hline $\mathrm{C} 29-\mathrm{C} 28-\mathrm{Br} 1$ & $118.1(4)$ \\
\hline C24-C29-H29 & 120.2 \\
\hline C28-C29-C24 & $119.6(4)$ \\
\hline C28-C29-H29 & 120.2 \\
\hline
\end{tabular}

Symmetry transformations used to generate equivalent atoms: 
Table 4. Anisotropic displacement parameters $\left(\AA^{2} \times 10^{3}\right)$ for 3ma. The anisotropic displacement factor exponent takes the form: $-2 p^{2}\left[h^{2} a^{* 2} U^{11}+\ldots+2 h k a^{*} b^{*} U^{12}\right]$

\begin{tabular}{|c|c|c|c|c|c|c|}
\hline & $\mathrm{U}^{11}$ & $\mathrm{U}^{22}$ & $\mathrm{U}^{33}$ & $\mathrm{U}^{23}$ & $\mathrm{U}^{13}$ & $\mathrm{U}^{12}$ \\
\hline $\mathrm{Br} 1$ & $76(1)$ & $57(1)$ & $46(1)$ & $23(1)$ & $14(1)$ & $13(1)$ \\
\hline S1 & $34(1)$ & $38(1)$ & $25(1)$ & $2(1)$ & $-4(1)$ & $1(1)$ \\
\hline $\mathrm{O} 1$ & $36(2)$ & $43(2)$ & $23(2)$ & $-2(1)$ & $-6(1)$ & 11(1) \\
\hline $\mathrm{O} 2$ & $52(2)$ & $55(2)$ & $30(2)$ & $-11(2)$ & $-8(2)$ & $18(2)$ \\
\hline $\mathrm{O} 3$ & $49(2)$ & $49(2)$ & $28(2)$ & $-3(1)$ & $-8(2)$ & $21(2)$ \\
\hline $\mathrm{N} 1$ & $33(2)$ & $31(2)$ & $24(2)$ & $0(2)$ & $-2(1)$ & $1(2)$ \\
\hline $\mathrm{C} 1$ & $29(2)$ & $35(2)$ & $26(2)$ & $1(2)$ & $-2(2)$ & $-7(2)$ \\
\hline $\mathrm{C} 2$ & $31(2)$ & $34(2)$ & $23(2)$ & $2(2)$ & $0(2)$ & $-4(2)$ \\
\hline $\mathrm{C} 3$ & $30(2)$ & $29(2)$ & $29(2)$ & $2(2)$ & $-4(2)$ & $-5(2)$ \\
\hline $\mathrm{C} 4$ & $30(2)$ & $32(2)$ & $25(2)$ & $1(2)$ & $-4(2)$ & $-3(2)$ \\
\hline C5 & $34(2)$ & $31(2)$ & $23(2)$ & $-1(2)$ & $0(2)$ & $-1(2)$ \\
\hline C6 & $35(2)$ & $36(2)$ & $28(2)$ & $-2(2)$ & $-5(2)$ & $3(2)$ \\
\hline C7 & $30(2)$ & $35(2)$ & $23(2)$ & $1(2)$ & $-1(2)$ & $2(2)$ \\
\hline $\mathrm{C} 8$ & $41(2)$ & $40(2)$ & $31(2)$ & $-2(2)$ & $6(2)$ & $-10(2)$ \\
\hline C9 & 63(3) & $47(3)$ & $22(2)$ & $-4(2)$ & $10(2)$ & $-7(3)$ \\
\hline $\mathrm{C} 10$ & $60(3)$ & $41(3)$ & $25(2)$ & $-6(2)$ & $-6(2)$ & $-1(2)$ \\
\hline $\mathrm{C} 11$ & $42(3)$ & $47(3)$ & $34(2)$ & $-6(2)$ & $-7(2)$ & $-7(2)$ \\
\hline $\mathrm{C} 12$ & $38(2)$ & $56(3)$ & $23(2)$ & $-2(2)$ & $-1(2)$ & $-8(2)$ \\
\hline $\mathrm{C} 13$ & $31(2)$ & $26(2)$ & $33(2)$ & $3(2)$ & $-1(2)$ & $-2(2)$ \\
\hline $\mathrm{C} 14$ & $34(2)$ & $32(2)$ & $32(2)$ & $2(2)$ & $-4(2)$ & $-5(2)$ \\
\hline $\mathrm{C} 15$ & $31(2)$ & $33(2)$ & $43(3)$ & $7(2)$ & $-4(2)$ & $1(2)$ \\
\hline $\mathrm{C} 16$ & $36(2)$ & $34(2)$ & $44(3)$ & $2(2)$ & $4(2)$ & $3(2)$ \\
\hline $\mathrm{C} 17$ & $44(3)$ & $37(2)$ & $35(3)$ & $-2(2)$ & $5(2)$ & $1(2)$ \\
\hline $\mathrm{C} 18$ & $37(2)$ & $33(2)$ & $31(2)$ & $2(2)$ & $-2(2)$ & $0(2)$ \\
\hline $\mathrm{C} 19$ & $30(2)$ & $31(2)$ & $25(2)$ & $2(2)$ & $0(2)$ & $-3(2)$ \\
\hline $\mathrm{C} 20$ & $32(2)$ & $34(2)$ & $25(2)$ & $3(2)$ & $-2(2)$ & $2(2)$ \\
\hline $\mathrm{C} 21$ & $52(3)$ & $44(3)$ & $40(3)$ & $-1(2)$ & $0(2)$ & $22(2)$ \\
\hline $\mathrm{C} 22$ & $109(5)$ & $71(4)$ & $51(4)$ & $-5(3)$ & $-21(4)$ & $53(4)$ \\
\hline $\mathrm{C} 23$ & $33(2)$ & $30(2)$ & $23(2)$ & $0(2)$ & $-4(2)$ & $0(2)$ \\
\hline $\mathrm{C} 24$ & $30(2)$ & $32(2)$ & $27(2)$ & $-2(2)$ & $-4(2)$ & $-3(2)$ \\
\hline $\mathrm{C} 25$ & $32(2)$ & $45(2)$ & $32(2)$ & $-6(2)$ & $-4(2)$ & $5(2)$ \\
\hline $\mathrm{C} 26$ & $30(2)$ & $64(3)$ & $31(2)$ & $-10(2)$ & $5(2)$ & $2(2)$ \\
\hline $\mathrm{C} 27$ & $45(3)$ & $49(3)$ & $27(2)$ & $-5(2)$ & $10(2)$ & $-12(2)$ \\
\hline $\mathrm{C} 28$ & $45(3)$ & $40(2)$ & $27(2)$ & $1(2)$ & $2(2)$ & $-3(2)$ \\
\hline $\mathrm{C} 29$ & $33(2)$ & $40(2)$ & $29(2)$ & $1(2)$ & $3(2)$ & $1(2)$ \\
\hline
\end{tabular}


Table 5. Hydrogen coordinates $\left(\mathrm{x} 10^{4}\right)$ and isotropic displacement parameters $\left(\AA^{2} \times 10^{3}\right)$ for $3 \mathbf{m a}$.

\begin{tabular}{|c|c|c|c|c|}
\hline & $\mathrm{x}$ & $\mathrm{y}$ & $\mathrm{z}$ & $\mathrm{U}(\mathrm{eq})$ \\
\hline H1 & 3452 & 5033 & 5862 & 36 \\
\hline H6A & 2943 & 6108 & 5555 & 39 \\
\hline H6B & 5557 & 6153 & 5327 & 39 \\
\hline $\mathrm{H} 8$ & 3073 & 5152 & 6922 & 45 \\
\hline H9 & 4336 & 5509 & 7886 & 53 \\
\hline H10 & 7794 & 6080 & 7980 & 51 \\
\hline H11 & 10008 & 6306 & 7098 & 49 \\
\hline H12 & 8760 & 5944 & 6135 & 47 \\
\hline H14 & 12768 & 3640 & 5593 & 39 \\
\hline H15 & 15820 & 2961 & 5305 & 43 \\
\hline H16 & 16339 & 2699 & 4265 & 46 \\
\hline H17 & 13786 & 3088 & 3521 & 46 \\
\hline H18 & 10647 & 3740 & 3813 & 40 \\
\hline $\mathrm{H} 21 \mathrm{~A}$ & -1889 & 7485 & 4476 & 54 \\
\hline $\mathrm{H} 21 \mathrm{~B}$ & -4069 & 7019 & 4535 & 54 \\
\hline $\mathrm{H} 22 \mathrm{~A}$ & -4379 & 7056 & 3441 & 115 \\
\hline $\mathrm{H} 22 \mathrm{~B}$ & -2482 & 7608 & 3449 & 115 \\
\hline $\mathrm{H} 22 \mathrm{C}$ & -4926 & 7719 & 3770 & 115 \\
\hline $\mathrm{H} 23 \mathrm{~A}$ & 1611 & 5089 & 3768 & 35 \\
\hline $\mathrm{H} 23 \mathrm{~B}$ & -526 & 5555 & 3671 & 35 \\
\hline $\mathrm{H} 25$ & 5285 & 5276 & 3189 & 43 \\
\hline $\mathrm{H} 26$ & 7011 & 5706 & 2309 & 50 \\
\hline $\mathrm{H} 27$ & 5246 & 6560 & 1780 & 49 \\
\hline $\mathrm{H} 29$ & -138 & 6510 & 2996 & 40 \\
\hline
\end{tabular}


Table 6. Torsion angles $\left[^{\circ}\right]$ for $3 \mathbf{m a}$.

\begin{tabular}{|c|c|}
\hline Br1-C28-C29-C24 & 178.1(3) \\
\hline $\mathrm{S} 1-\mathrm{C} 2-\mathrm{C} 4-\mathrm{O} 1$ & $178.5(3)$ \\
\hline $\mathrm{S} 1-\mathrm{C} 2-\mathrm{C} 4-\mathrm{N} 1$ & $-0.3(5)$ \\
\hline S1-C3-C13-C14 & $-6.2(6)$ \\
\hline S1-C3-C13-C18 & $175.2(3)$ \\
\hline $\mathrm{O} 1-\mathrm{C} 5-\mathrm{C} 6-\mathrm{C} 1$ & $-46.8(5)$ \\
\hline O1-C5-C19-C20 & $-174.3(3)$ \\
\hline O1-C5-C19-C23 & $5.3(5)$ \\
\hline N1-C3-C13-C14 & $172.2(4)$ \\
\hline N1-C3-C13-C18 & $-6.4(6)$ \\
\hline $\mathrm{C} 1-\mathrm{C} 2-\mathrm{C} 4-\mathrm{O} 1$ & $-1.0(7)$ \\
\hline $\mathrm{C} 1-\mathrm{C} 2-\mathrm{C} 4-\mathrm{N} 1$ & $-179.8(4)$ \\
\hline C1-C7-C8-C9 & $-178.9(4)$ \\
\hline $\mathrm{C} 1-\mathrm{C} 7-\mathrm{C} 12-\mathrm{C} 11$ & $178.6(4)$ \\
\hline C2-S1-C3-N1 & $-0.2(3)$ \\
\hline C2-S1-C3-C13 & 178.3(3) \\
\hline C2-C1-C6-C5 & $50.2(5)$ \\
\hline $\mathrm{C} 2-\mathrm{C} 1-\mathrm{C} 7-\mathrm{C} 8$ & $-125.2(4)$ \\
\hline $\mathrm{C} 2-\mathrm{C} 1-\mathrm{C} 7-\mathrm{C} 12$ & $55.0(6)$ \\
\hline C3-S1-C2-C1 & 179.7(4) \\
\hline $\mathrm{C} 3-\mathrm{S} 1-\mathrm{C} 2-\mathrm{C} 4$ & $0.2(3)$ \\
\hline C3-N1-C4-O1 & $-178.8(3)$ \\
\hline C3-N1-C4-C2 & $0.1(5)$ \\
\hline C3-C13-C14-C15 & $-179.2(4)$ \\
\hline C3-C13-C18-C17 & $178.0(4)$ \\
\hline C4-O1-C5-C6 & $15.3(5)$ \\
\hline C4-O1-C5-C19 & $-166.8(4)$ \\
\hline C4-N1-C3-S1 & $0.1(4)$ \\
\hline C4-N1-C3-C13 & $-178.4(4)$ \\
\hline C5-O1-C4-N1 & $-171.6(3)$ \\
\hline $\mathrm{C} 5-\mathrm{O} 1-\mathrm{C} 4-\mathrm{C} 2$ & $9.6(6)$ \\
\hline C5-C19-C20-O2 & $-12.9(7)$ \\
\hline $\mathrm{C} 5-\mathrm{C} 19-\mathrm{C} 20-\mathrm{O} 3$ & $167.2(4)$ \\
\hline C5-C19-C23-C24 & $-89.1(5)$ \\
\hline C6-C1-C2-S1 & $151.9(3)$ \\
\hline C6-C1-C2-C4 & $-28.7(5)$ \\
\hline C6-C1-C7-C8 & $113.8(4)$ \\
\hline C6-C1-C7-C12 & $-66.0(5)$ \\
\hline C6-C5-C19-C20 & $3.3(7)$ \\
\hline C6-C5-C19-C23 & $-177.1(4)$ \\
\hline C7-C1-C2-S1 & $27.5(6)$ \\
\hline
\end{tabular}




$\begin{array}{lc}\text { C7-C1-C2-C4 } & -153.1(4) \\ \text { C7-C1-C6-C5 } & 176.5(4) \\ \text { C7-C8-C9-C10 } & -0.4(7) \\ \text { C8-C7-C12-C11 } & -1.2(7) \\ \text { C8-C9-C10-C11 } & 0.1(7) \\ \text { C9-C10-C11-C12 } & -0.3(8) \\ \text { C10-C11-C12-C7 } & 0.9(8) \\ \text { C12-C7-C8-C9 } & 1.0(7) \\ \text { C13-C14-C15-C16 } & 1.3(6) \\ \text { C14-C13-C18-C17 } & -0.6(6) \\ \text { C14-C15-C16-C17 } & -0.8(6) \\ \text { C15-C16-C17-C18 } & -0.3(7) \\ \text { C16-C17-C18-C13 } & 1.1(7) \\ \text { C18-C13-C14-C15 } & -0.6(6) \\ \text { C19-C5-C6-C1 } & 135.7(4) \\ \text { C19-C23-C24-C25 } & 93.7(5) \\ \text { C19-C23-C24-C29 } & -89.4(5) \\ \text { C20-O3-C21-C22 } & 167.0(5) \\ \text { C20-C19-C23-C24 } & 90.5(5) \\ \text { C21-O3-C20-O2 } & 4.5(6) \\ \text { C21-O3-C20-C19 } & -175.5(4) \\ \text { C23-C19-C20-O2 } & 167.5(4) \\ \text { C23-C19-C20-O3 } & -12.4(5) \\ \text { C23-C24-C25-C26 } & 176.8(4) \\ \text { C23-C24-C29-C28 } & -177.5(4) \\ \text { C24-C25-C26-C27 } & 1.0(7) \\ \text { C25-C24-C29-C28 } & -0.6(6) \\ \text { C25-C26-C27-C28 } & -1.2(7) \\ \text { C26-C27-C28-Br1 } & -177.2(3) \\ \text { C26-C27-C28-C29 } & 0.5(7) \\ \text { C27-C28-C29-C24 } & 0.4(7) \\ \text { C29-C24-C25-C26 } & -0.1(6)\end{array}$

Symmetry transformations used to generate equivalent atoms:

Table 7. Hydrogen bonds for $3 \mathrm{ma}\left[\AA\right.$ and $\left.^{\circ}\right]$.

\begin{tabular}{lllll}
\hline $\mathrm{D}-\mathrm{H} \ldots \mathrm{A}$ & $\mathrm{d}(\mathrm{D}-\mathrm{H})$ & $\mathrm{d}(\mathrm{H} \ldots \mathrm{A})$ & $\mathrm{d}(\mathrm{D} \ldots \mathrm{A})$ & $<$ (DHA)
\end{tabular}

